SF 235.

.P5

Copy 1

A Handbook

of

Dairy Statistics

By T. R. PIRTLE, Dairy Division

क
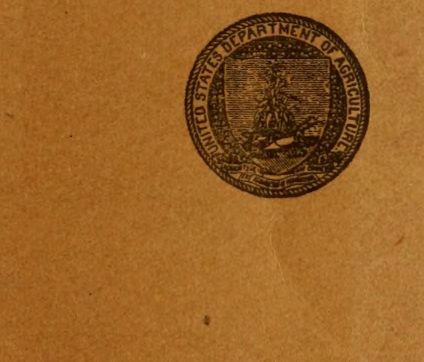

UNTTED STATES

DEPARTMENT OF AGRICULTURE

Contribution from the Bureau of Animal Industry JOHN R. MOHLER, Chief

Washington, D. C.

June, 1922 



\section{A Handbook}

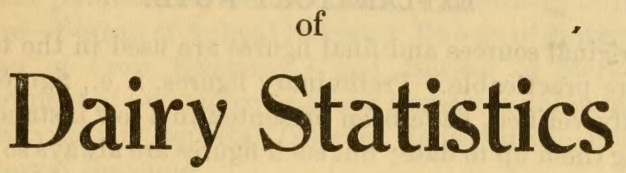

By T. R. PIRTLE, Dairy Division

is

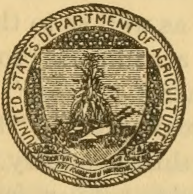

UNITED STATES

DEPARTMENT OF AGRICULTURE

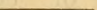

Contribution from the Bureau of Animal Industry JOHN R. MOHLER, Chief

Washington, D. C.

June, 1922

GOVERNMENT PRINTINQ OFFICE : 1022 


\section{A HANDBOOK OF DAIRY STATISTICS.}

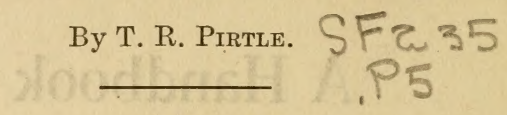

EXPLANATORY NOTE.

Original sources and final figures are used in the tables where practicable. Preliminary figures, i. e., figures not finally verified, have been presented in a few instances to bring them up to date; but such figures are always so indicated in the tables. Many countries have found it necessary to revise the statistics gathered during the war, especially those relating to the number of cattle. During the war preliminary figures were not always indicated, but later checking up has shown them to be approximately correct, although the final figures vary from them to some extent.

(YB.) used in connection with a table indicates that the table may be continued to cover the latest figures available by reference to the Yearbook of the United States Department of Agriculture, as well as to the original source.

"Special imports" are imports brought into a country for consumption. "General imports" are for all purposes, and a part may be reexported. "Domestic exports" are of goods produced within a country.

Units of weight used to convert foreign or metric weights to pounds, United States, are:

Pounds.

1 kilogram

2. 2046

1 quintal

220.46

1 poud (Russian).

36.1128

1 hundredweight (English)

1pound (Spanish pound for Cuba).

1.0161

The terms "dairy cows" and "cows" do not mean the same, the latter term including cows not used for dairy purposes. The number of "cows" was used when the number of "dairy cows" was not obtainable.

Where blank spaces are left in tables records were not made or were not available.

When figures are given for any year it may be assumed that it is the calendar year unless otherwise stated, although L'BNAR' 2 K OF CONGRESS RECEIVED AUG 151922 DOCUMENTES DIVISION 
some of the authorities are indefinite on this point. Where the fiscal year is used, the date of ending is often changed in the middle of the series of years given.

The sources of the data quoted from United States Government publications are as follows: Department of Agriculture-Bureau of Animal Industry, Bureau of Statistics, ${ }^{1}$ Bureau of Crop Estimates, ${ }^{2}$ and Bureau of Markets; ${ }^{2}$ Department of Commerce - Census Bureau and Commerce and Navigation Reports; Treasury Department-Bureau of Internal Revenue.

Reconstruction of territory recognized in all countries, 1919 and later.

1 Bureau of Statistics is a former designation for the Bureau of Crop Estimates.

2 The Bureau of Markets and the Bureau of Crop Estimates were consolidated July 1, 1921, and are now known as the "Bureau of Markets and Crop Estimates."

THE STATISTICS of an industry show its trend and form a basis for action toward it best development. Because data regarding dairy products and the trade in dairy products have never been jrought together for convenient reference, attention has not been given to the trend of dairying in the degree that its importance warrants. To remedy this condition this volume has been prepared. In it will be found the available statistics on dairy production and trade which may be of value to the dairy industry. 
GENERAL STATISTICS.

\section{TABLE 1.-POPULATION of various countries.}

[Statesman's Year Book.]

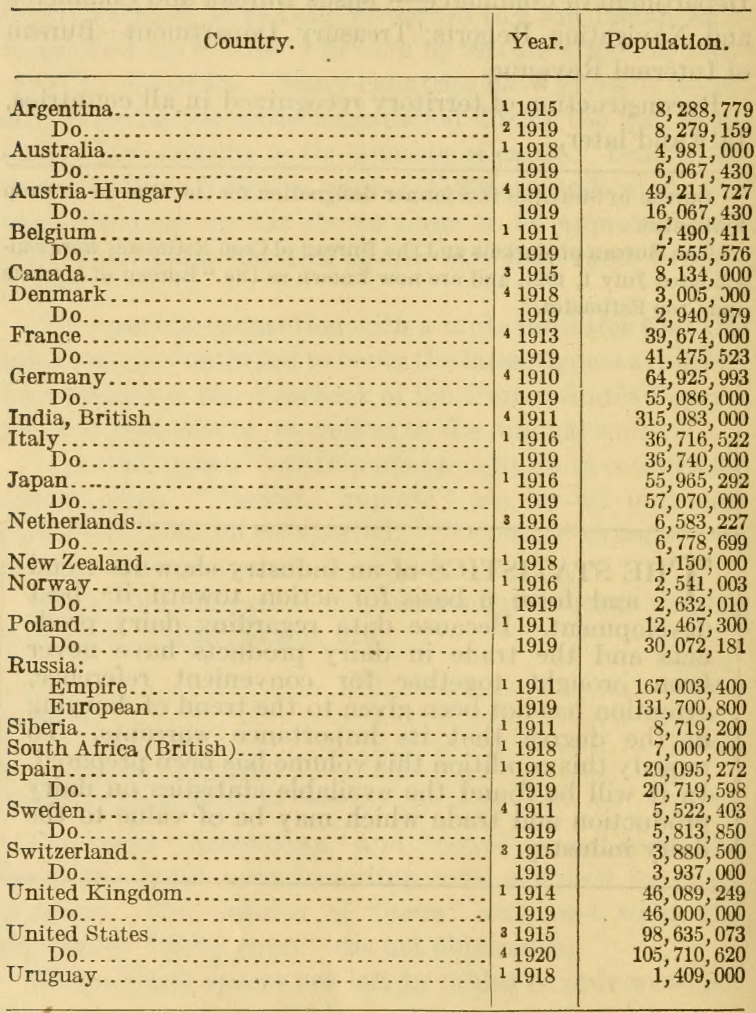

1 Estimate.

2 Source or method of estimate not stated for 1919.

3 Prorated.

4 Census. 


\section{TABLE 2.-Average YIELD of MILK per year of milk cows in various countries.}

[Various sources.]

\begin{tabular}{|c|c|c|c|c|c|}
\hline Country. & Year. & $\begin{array}{l}\text { Average } \\
\text { yield of } \\
\text { milk. }\end{array}$ & Country. & Yeăr. & $\begin{array}{l}\text { A verage } \\
\text { yield of } \\
\text { milk. }\end{array}$ \\
\hline 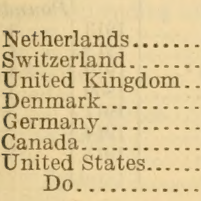 & $\begin{array}{l}1902 \\
1914 \\
1914 \\
1914 \\
1912 \\
1911 \\
1917 \\
1920\end{array}$ & $\begin{array}{r}\text { Pounds. } \\
7,585 \\
6,950 \\
5,934 \\
5,666 \\
4,350 \\
3,779 \\
3,716 \\
3,627\end{array}$ & 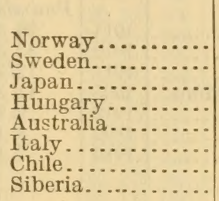 & $\begin{array}{l}1910 \\
1911 \\
1918 \\
1914 \\
1916 \\
1914 \\
1916 \\
1916\end{array}$ & $\begin{array}{r}\text { Pounds. } \\
3,680 \\
3,600 \\
3,339 \\
2,932 \\
2,719 \\
2,279 \\
1,520 \\
1,192\end{array}$ \\
\hline
\end{tabular}

\section{TABLE 3.-Average per capita CONSUMPTION of} WHOLE MILK in various countries per year.

[Numerous authorities.]

\begin{tabular}{|c|c|c|c|c|c|}
\hline Country. & Year. & $\begin{array}{c}\text { Milk } \\
\text { con- } \\
\text { sumed. }\end{array}$ & Country. & Year. & $\begin{array}{c}\text { Milk } \\
\text { con- } \\
\text { sumed. }\end{array}$ \\
\hline 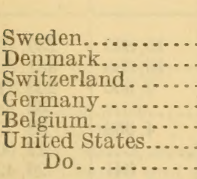 & $\begin{array}{l}1914 \\
1914 \\
1914 \\
1913 \\
1895 \\
1917 \\
1920\end{array}$ & $\begin{array}{r}\text { Gallons. } \\
69.7 \\
68.5 \\
67.4 \\
61.0 \\
44.0 \\
42.4 \\
43.0\end{array}$ & $\begin{array}{l}\text { Netherlands........ } \\
\text { Canada............. } \\
\text { Hungary } \ldots . . . . . \\
\text { United Kingdom. } \\
\text { Italy ............... } \\
\text { Hawai............ }\end{array}$ & $\begin{array}{l}1902 \\
1916 \\
1914 \\
1914 \\
1913 \\
1916\end{array}$ & $\begin{array}{r}\text { Gallons. } \\
39.5 \\
26.0 \\
24.3 \\
22.2 \\
4.2 \\
1.0\end{array}$ \\
\hline
\end{tabular}

\section{TABLE 4.-Average per capita CONSUMPTION of} BUTTER in various countries per year.

[Numerous authorities.]

\begin{tabular}{|c|c|c|c|c|c|}
\hline Country. & Year. & $\begin{array}{c}\text { Butter } \\
\text { con- } \\
\text { sumed. }\end{array}$ & Country. . & Year. & $\begin{array}{c}\text { Butter } \\
\text { con- } \\
\text { sumed. }\end{array}$ \\
\hline 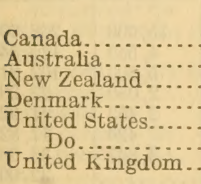 & $\begin{array}{l}1911 \\
1913 \\
1914 \\
1914 \\
1909 \\
1919 \\
1914\end{array}$ & $\begin{array}{r}\text { Pounds. } \\
27.7 \\
25.6 \\
21.7 \\
19.0 \\
17.5 \\
15.5 \\
17.0\end{array}$ & 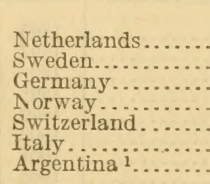 & $\begin{array}{l}1917 \\
1917 \\
1913 \\
1906 \\
1914 \\
1913 \\
1913\end{array}$ & $\begin{array}{r}\text { Pounds. } \\
16.8 \\
16.5 \\
14.7 \\
14.0 \\
12.1 \\
2.5 \\
1.8\end{array}$ \\
\hline
\end{tabular}

\footnotetext{
1 Factory butter only.
} 


\section{TABLE 5.-Average per capita CONSUMPTION of CHEESE in various countries per year.}

[Numerous authorities.]

\begin{tabular}{|c|c|c|c|c|c|}
\hline Country. & Year. & $\begin{array}{c}\text { Cheese } \\
\text { con- } \\
\text { sumed. }\end{array}$ & Country. & Year. & $\begin{array}{c}\text { Cheese } \\
\text { con- } \\
\text { sumed. }\end{array}$ \\
\hline Switzerlan & 1914 & $\begin{array}{r}\text { Pounds. } \\
26.4\end{array}$ & Italy & 1913 & Pounds. \\
\hline Netherlan & 1912 & 13.3 & Australia..... & 1913 & 4.8 \\
\hline Denmark. & 1914 & 12.3 & United States & 1919 & 24.2 \\
\hline United Kingdom. & 1905 & 11.2 & Do. ${ }^{1} \ldots \ldots$ & 1909 & 3.8 \\
\hline Germany.............. & 1912 & 9. & Canada,..... & 1911 & 3.0 \\
\hline France... & 1892 & 8.1 & New Zealand & 1914 & 3.0 \\
\hline Norway. & 1910 & 7.1 & Argentina ${ }^{3} \ldots$ & 1913 & 2.9 \\
\hline
\end{tabular}

${ }^{1}$ Census. $\quad{ }^{2}$ Including cottage cheese. $\quad{ }^{3}$ Factory cheese only.

TABLE 6.-Trend of COW-TESTING ASSOCIATIONS.

[Cooperative Danish agricultural societies and Canada News Letter, No. $15,1920$.

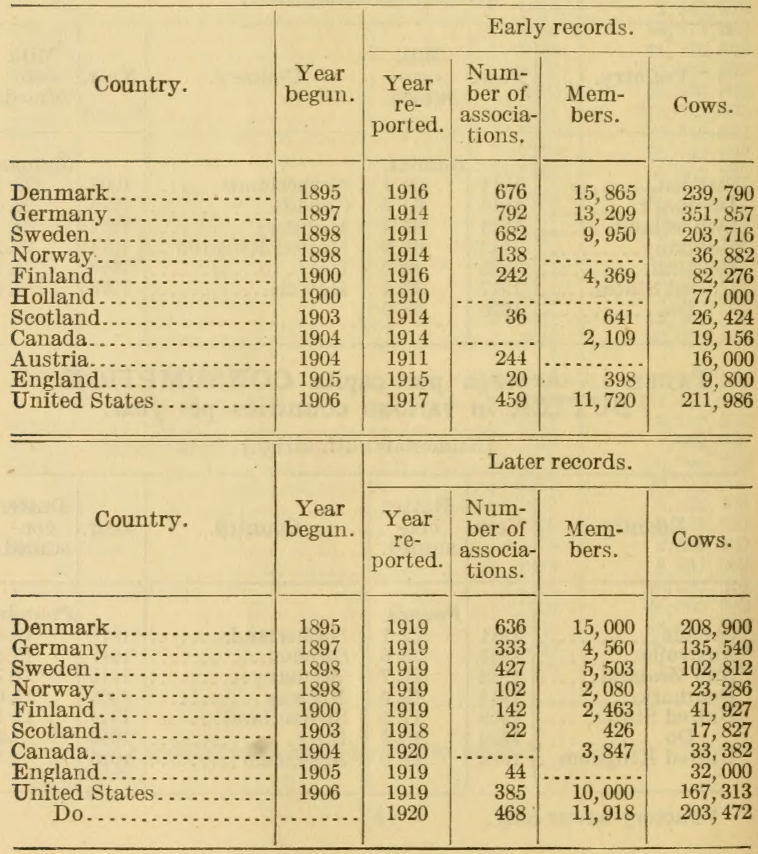




\section{TABLE 7.-PRODUCTION and EXPORTATION of BUTTER for various countries.}

[Numerous authoritiès.]

\begin{tabular}{|c|c|c|c|}
\hline Country. & Year. & Production. & Exports. \\
\hline United States & 1909 & $\begin{array}{l}\text { Pounds. } \\
\text { 1.621.000.000 }\end{array}$ & $\begin{array}{l}\text { Pounds. } \\
5,981.000\end{array}$ \\
\hline Do............. & 1919 & $1,648,505,382$ & $34,556,485$ \\
\hline Germany. & 1913 & $881,840,000$ & 602,000 \\
\hline Russia (including Siberia) & 1913 & $326,253,000$ & $172,003,000$ \\
\hline & 1914 & $286,598,000$ & $44,619,000$ \\
\hline & 1914 & 257 , & $210,084,000$ \\
\hline $\mathrm{Aus}$ & 1916 & 9,000 & $16,722,000$ \\
\hline Can & 1911 & 201,8 & $3,142,000$ \\
\hline & 1908 & 1177,0 & $1,125,000$ \\
\hline Net & 1912 & $147,835,000$ & $86,307,000$ \\
\hline Ita & 1914 & ${ }^{2} 110,000,000$ & $9,310,000$ \\
\hline New Zealand & 1916 & $63,273,000$ & $40,167,000$ \\
\hline & 1916 & $262,135,000$ & $28,704,000$ \\
\hline Switz & 1916 & $37,000,000$ & 2,646 \\
\hline & 1914 & $20,518,000$ & $7,676,000$ \\
\hline Union of South Africa. & 1916 & $15,980,000$ & \\
\hline
\end{tabular}

${ }^{1}$ Factory butter only, for Ireland. $\quad{ }^{2}$ Factory butter only. ${ }^{3}$ None.

\section{TABLE 8.-Approximate amount of MILK SOLIDS in dairy products.}

[Various authorities.]

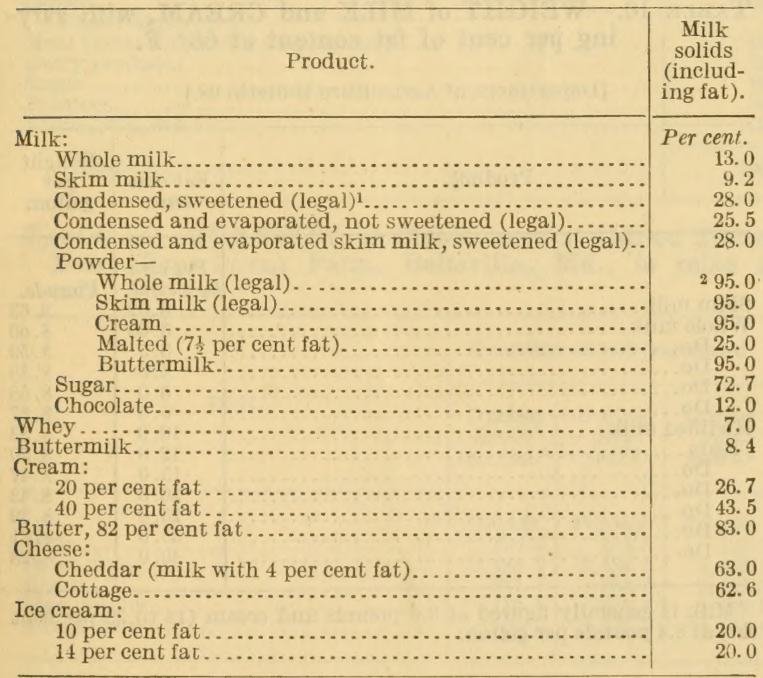

1 U. S. Dept. of Agriculture, B. A. I. Cir. 36.

${ }^{2}$ The moisture of whole and skimmed-milk powder commercially is about 2 per cent. 
TABLE 9.-STANDARDS of BUTTERFAT and limit of MOISTURE in BUTTER in various countries.

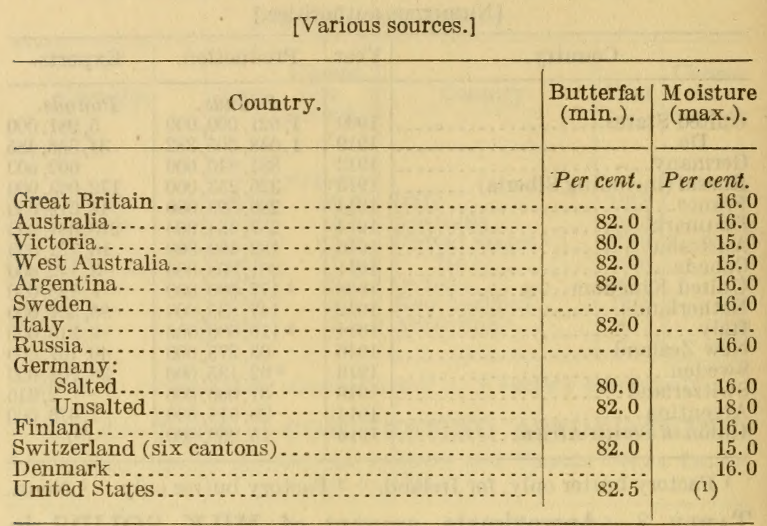

1 Below 16.

TABLE 10.-WEIGHT of MILK and CREAM, with varying per cent of fat content at $68^{\circ} \mathrm{F}$.

[Department of Agriculture Bulletin 98.]

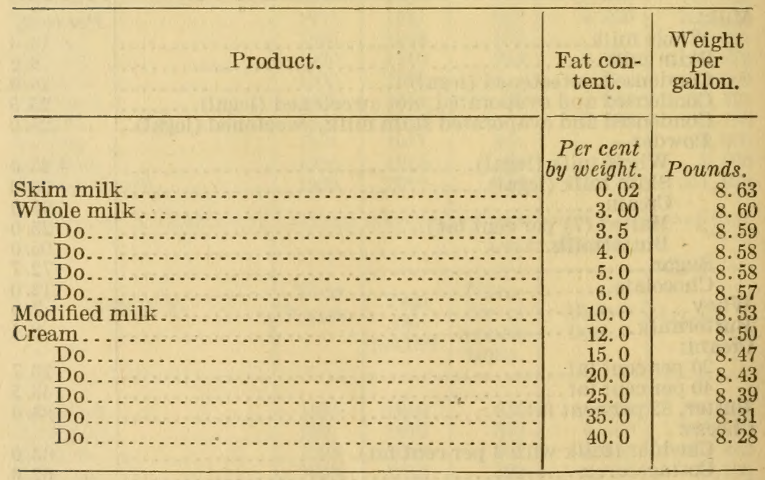

Milk is generally figured at 8.6 pounds and cream (18 to 20 per cent fat) at 8.4 pounds per gallon. 
TABLE 11.-PROPORTION Of YEARLY BUTTERFAT RECEIPTS, received monthly by creameries in Iowa, Minnesota, and Wisconsin, for the years 1913 to 1916.

[Dairy Division, Bureau of Animal Industry.]

\begin{tabular}{|c|c|c|c|}
\hline Month. & Butterfat. & Month. & Butterfat. \\
\hline 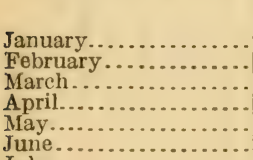 & \begin{tabular}{|r|} 
Per cent. \\
6.53 \\
6.46 \\
7.11 \\
7.45 \\
10.71 \\
12.33
\end{tabular} & 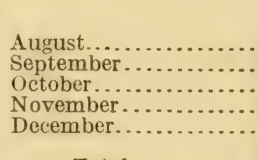 & $\begin{array}{r}\text { Per cent. } \\
9.81 \\
8.65 \\
7.53 \\
6.07 \\
6.09\end{array}$ \\
\hline July ............................. & 11.26 & Total. & 100.00 \\
\hline
\end{tabular}

TABLE 12.-NORMAL FOOD for the United Kingdom.

[Royal Society Food Comm., Mar. 30, 1917.]

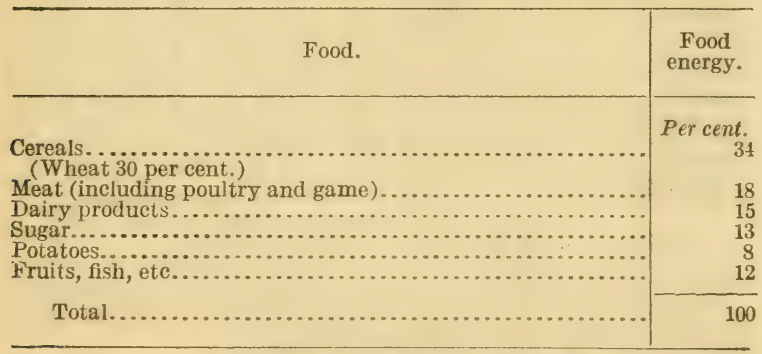

TABLE 13.-QUANTITY of MILK used at United States Dairy Experiment Farm, Beltsville, Md., to raise a calf.

\begin{tabular}{|c|c|c|c|}
\hline \multirow[b]{2}{*}{ Feed. } & \multicolumn{3}{|c|}{ Quantity of feed consumed. } \\
\hline & $\begin{array}{l}\text { During } \\
\text { first } 6 \\
\text { months. }\end{array}$ & $\begin{array}{c}\text { During } \\
\text { first } \\
\text { year.1 }\end{array}$ & $\begin{array}{c}\text { During } \\
\text { two } \\
\text { years. }\end{array}$ \\
\hline 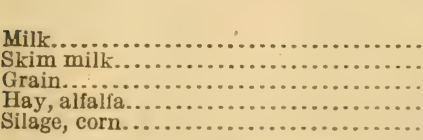 & $\begin{array}{r}\text { Pounds. } \\
125.3 \\
2,486.1 \\
317.7 \\
112.1 \\
72 \delta .5\end{array}$ & $\begin{array}{r}\text { Pounds. } \\
125.3 \\
2,486.1 \\
883.3 \\
566.1 \\
3,647.5\end{array}$ & $\begin{array}{r}\text { Pounds. } \\
125.3 \\
2,486.1 \\
2,107.3 \\
1,599.1 \\
11,164.6\end{array}$ \\
\hline
\end{tabular}

1 A verage of 12 calves.

2 Average of 11 calves; 4 of the calves were purebred or grade Holsteins and 7 were purebred or grade Guernseys. Average live weight at two years, 861 pounds.

$$
75578^{\circ}-22-2
$$




\section{UNITED STATES STATISTICS.}

\section{TABLE 14.-Number and percentages of CATTLE of various BREEDS in the United States, 1920.}

[Bureau of Markets and Crop Estimates.]

\begin{tabular}{|c|c|c|c|c|c|c|c|c|}
\hline Cattle. & 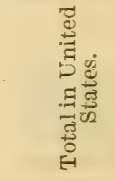 & & 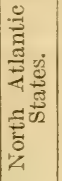 & 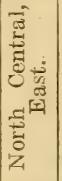 & 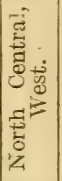 & 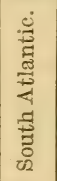 & 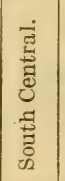 & 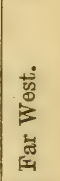 \\
\hline & Number. & P. ct. & $P . c t$. & $\begin{array}{r}P . c t . \\
3.6\end{array}$ & P.ct. & P.ct. & P.ct. & P.ct. \\
\hline & $\begin{array}{l}479,000 \\
412,000\end{array}$ & $\begin{array}{r}3.0 \\
.6\end{array}$ & $\begin{array}{l}0.3 \\
4.8\end{array}$ & $\begin{array}{r}3.6 \\
.4\end{array}$ & $\begin{array}{r}6.9 \\
.3\end{array}$ & $\begin{array}{r}2.9 \\
.3\end{array}$ & 3.1 & 0.8 \\
\hline & & .3 & .4 & .7 & .2 & & & \\
\hline & & & .4 & .1 & .1 & .7 & .3 & .4 \\
\hline & & 2 & .4 & .1 & .1 & .1 & .7 & \\
\hline & & & .1 & & 1.8 & .2 & .3 & \\
\hline & 1,993, & 2.9 & 8.8 & 6.3 & 1.9 & 3.6 & .4 & 1.3 \\
\hline & 14 & 21.0 & 1.7 & 7.8 & 24.2 & 9.1 & 22.5 & 40.9 \\
\hline & & 16.2 & 54.0 & 29.3 & 8.7 & 9.0 & 5.2 & 14.9 \\
\hline & 9,55 & 14.0 & 14.3 & 15.3 & 4.8 & 29.5 & 23.8 & 8.8 \\
\hline & & 1.5 & .1 & 1.8 & 1.9 & & 2.4 & . \\
\hline Red & 1,772, & 2.6 & .2 & 1.7 & 3.3 & 1.6 & 5.4 & \\
\hline & 15,429 & 22.6 & 5.4 & 23.3 & 36.2 & 9.5 & 15.9 & 21.9 \\
\hline & & 3.1 & 2.2 & 2.1 & 2.3 & 6.8 & 4.9 & 1.8 \\
\hline ondes & & 10.3 & 6.9 & 6.8 & 7.3 & 26.2 & 15.0 & 7.2 \\
\hline Total.. & $68,230,000$ & 100.0 & 100.0 & 100.0 & 100.0 & 100.0 & 100.0 & 100.0 \\
\hline
\end{tabular}

The information was brought out by an inquiry sent to 14,000 special livestock reporters of the bureau. Grades and serubs were included in the breed in which the type predominated.

TABLE 15.-Number and value of CATTLE EXPORTED from the United States.

[Commerce and Navigation Reports; · (YB.).]

\begin{tabular}{|c|c|c|c|c|c|}
\hline \multirow{2}{*}{$\begin{array}{c}\text { Year ended } \\
\text { June } 30 .\end{array}$} & \multicolumn{2}{|c|}{ Cattle. } & \multirow{2}{*}{$\begin{array}{c}\text { Year ended } \\
\text { June } 30 .\end{array}$} & \multicolumn{2}{|c|}{ Cattle. } \\
\hline & Number. & Value. & & Number. & Value. \\
\hline 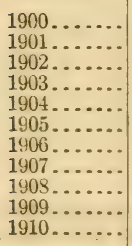 & $\begin{array}{l}397,286 \\
459,218 \\
392,884 \\
402,178 \\
593,409 \\
567,806 \\
581,239 \\
423,051 \\
349,210 \\
207,542 \\
139,430\end{array}$ & $\begin{array}{r}\$ 30,635,153 \\
37,566,980 \\
29,902,212 \\
29,848,936 \\
42,256,291 \\
40,598,048 \\
42,081,170 \\
34,577,392 \\
29,339,134 \\
18,046,976 \\
12,200,154\end{array}$ & $\begin{array}{l}1911 \ldots \ldots . \\
1912 \ldots \ldots \\
1913 \ldots \ldots \\
1914 \ldots \ldots \\
1915 \ldots \ldots \\
1916 \ldots \ldots \\
1917 \ldots \ldots \\
1918 \ldots \ldots \\
1919 \ldots \ldots \\
1920 \ldots \ldots \\
1921 \ldots \ldots \\
\end{array}$ & $\begin{array}{r}150,100 \\
105,506 \\
24,714 \\
18,376 \\
5,484 \\
21,287 \\
13,387 \\
18,213 \\
42,345 \\
83,039 \\
145,623\end{array}$ & $\begin{array}{r}\$ 13,163,920 \\
8,870,075 \\
1,177,199 \\
647,288 \\
702,847 \\
2,378,248 \\
949,503 \\
1,247,800 \\
2,092,816 \\
11,921,518 \\
10,950,507\end{array}$ \\
\hline
\end{tabular}


TABLE 16.-Number of DAIRY COWS and OTHER CATTLE on farms in the United States from 1840 to 1922 .

[Census Bureau and Bureau of Markets and Crop Estimates.]

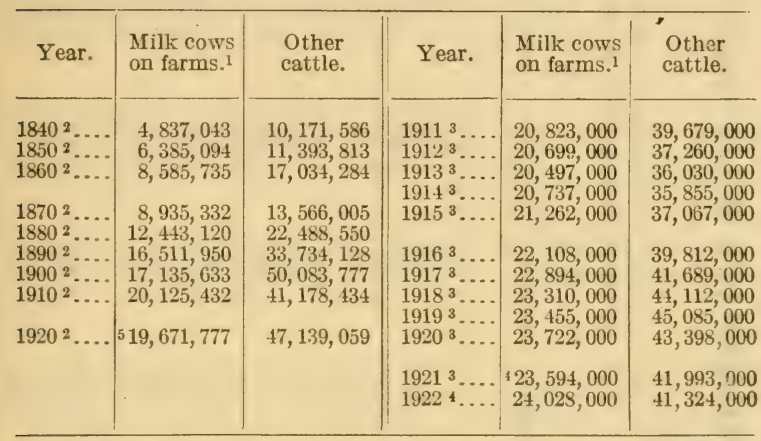

1 The number of cows within towns and villages amounts to $2,000,000$. 2 Census. The census report figures are for June 1, except for 1910, which is for April 15; and 1920, which was for January 1.

3 Estimates, January 1.

4 Preliminary.

5 Dairy breeds only.

\section{TABLE 17.-Kind and number of PUREBRED CATTLE} imported into the United States from 1885 to 1920, inclusive.

[Bureau of Animal Industry.]

\begin{tabular}{|c|c|c|c|}
\hline Breed. & Number. & Breed. & Number. \\
\hline 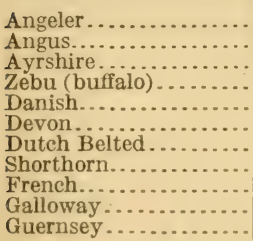 & $\begin{array}{r}14 \\
750 \\
1,762 \\
44 \\
1 \\
51 \\
69 \\
2,491 \\
62 \\
504 \\
10,114\end{array}$ & 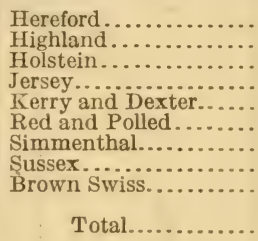 & $\begin{array}{r}799 \\
19 \\
1,864 \\
6,304 \\
243 \\
520 \\
24 \\
35 \\
83\end{array}$ \\
\hline
\end{tabular}

The figures for 1919 and 1920 include only those brought from overseas, as data on purebreds from Canada are not available. Records of importations from Canada were incomplete for a large portion of this period. 


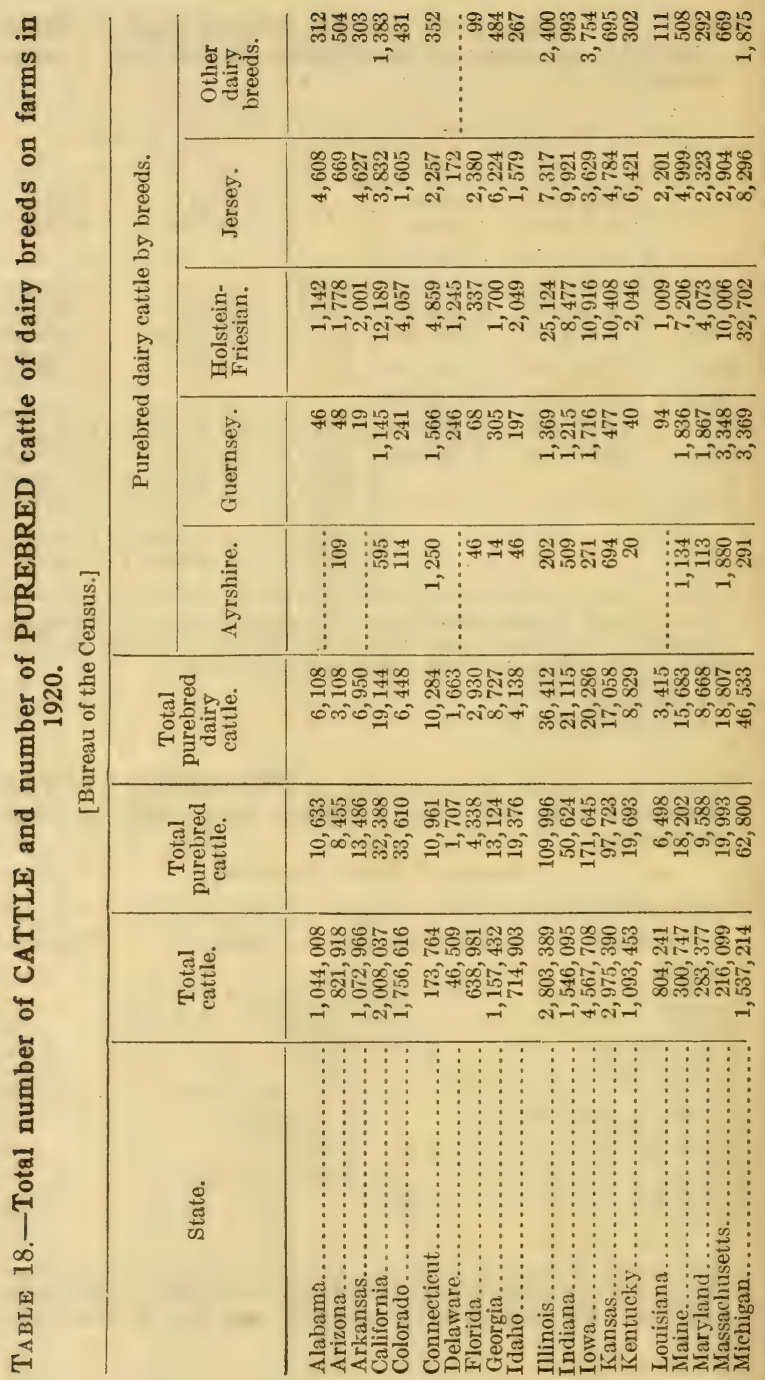




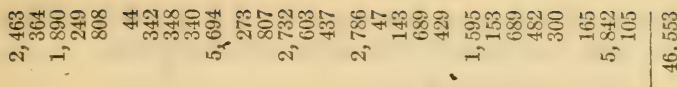

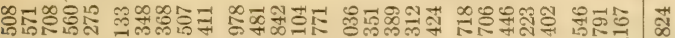

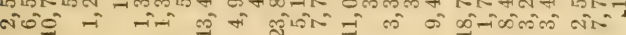
สี

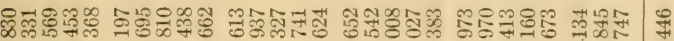

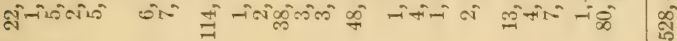

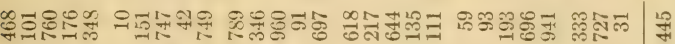

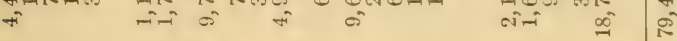

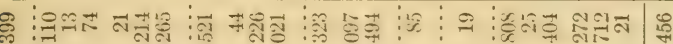
$\rightarrow 0 \rightarrow$ क $\rightarrow$ क

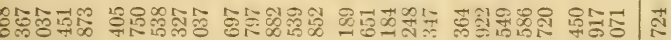

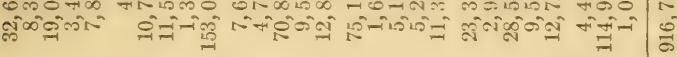

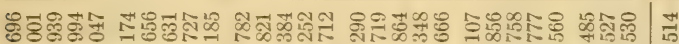

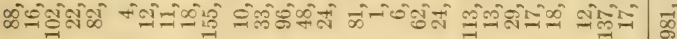

西

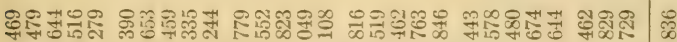
बiी

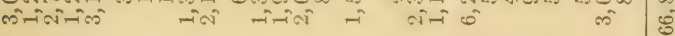

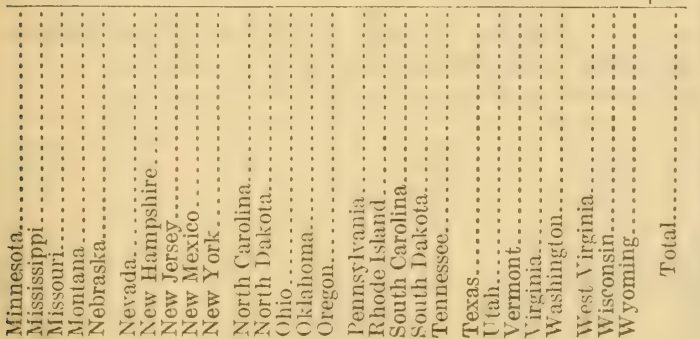


TABLE 19.-Number and value of CATTLE IMPORTED into the United States for all purposes.

[Commerce and Navigation Reports; (YB.).]

\begin{tabular}{|c|c|c|c|c|c|}
\hline \multirow{2}{*}{$\begin{array}{c}\text { Year ended } \\
\text { June } 30 .\end{array}$} & \multicolumn{2}{|c|}{ Cattle. } & \multirow{2}{*}{$\begin{array}{l}\text { Year ended } \\
\text { June } 30 \text {. }\end{array}$} & \multicolumn{2}{|c|}{ Cattle. } \\
\hline & Number. & Value. & & Number. & Value. \\
\hline 900 & 181,006 & $\$ 2,257,694$ & 1911. & 182,923 & $\$ 2,953,077$ \\
\hline & 146,022 & $1,931,433$ & 1912 . & 318,372 & $4,805,574$ \\
\hline 190 & 96,027 & $1,608,722$ & 1913. & 421,649 & $6,640,668$ \\
\hline & 66,175 & $1,161,548$ & 191 & 868,368 & $18,696,718$ \\
\hline & 16,056 & 310,737 & & 538, & 17, 513, \\
\hline 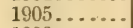 & 278 & & & 439, & $15,187,593$ \\
\hline & 29,0 & 548, & & 374, & $13,021,2$ \\
\hline & 32,402 & 565,12 & & 293,7 & $17,852,176$ \\
\hline & 92,356 & $1,507,31$ & & 440,3 & $36,995,921$ \\
\hline & 139,184 & $1,999,42$ & 1920. & 575,328 & $45,081,179$ \\
\hline & 195,938 & $2,999,824$ & 1921.. & 329,974 & $23,634,361$ \\
\hline
\end{tabular}

TABLE 20.-Number of cows in the United States which have produced 600 or more pounds of butterfat in a year prior to 1920 , in the principal dairy breeds.

[Herdbooks of cattle clubs.]

\begin{tabular}{|c|c|c|c|c|}
\hline Butterfat produced. & $\begin{array}{l}\text { Holstein- } \\
\text { Friesian. }\end{array}$ & Jersey. & $\begin{array}{c}\text { Guern- } \\
\text { sey. }\end{array}$ & Ayrshire. ${ }^{1}$ \\
\hline 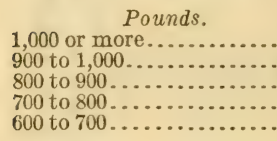 & $\begin{array}{r}27 \\
52 \\
126 \\
236 \\
590\end{array}$ & $\begin{array}{r}2 \\
15 \\
53 \\
148 \\
489\end{array}$ & $\begin{array}{r}2 \\
8 \\
27 \\
93 \\
297\end{array}$ & $\begin{array}{r}0 \\
2 \\
12 \\
25 \\
77\end{array}$ \\
\hline
\end{tabular}

${ }^{1}$ Complete to Apr. 15, 1921.

The table shows the number of cows, at their highest vield, with the butterfat produced for the year. That is, 52 Holstein, 15 jersey, 8 Guernsey, and 2 Ayrshire cows of record have produced 900 to 1,000 pounds of butterfat per cow in a year.

TABLE 21.-Number of cattle registered by the Ayrshire Breeders' Association from 1910 to Dec. 31, 1921.

[Secretary of the Ayrshire Breeders' Association.]

\begin{tabular}{|c|c|c|c|}
\hline Year. & $\begin{array}{l}\text { Bulls } \\
\text { and } \\
\text { cows. }\end{array}$ & Year. & $\begin{array}{l}\text { Bulls } \\
\text { and } \\
\text { cows. }\end{array}$ \\
\hline 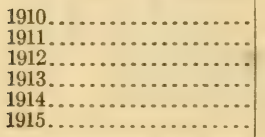 & $\begin{array}{l}3,233 \\
4,798 \\
2,884 \\
3,950 \\
4,912 \\
4,439\end{array}$ & $\begin{array}{l}1916 \ldots \ldots \ldots \\
1917 \ldots \ldots \ldots \\
1918 \ldots \ldots \ldots \\
1919 \ldots \ldots \ldots \\
1920 \ldots \ldots \\
1921 \ldots \ldots\end{array}$ & $\begin{array}{l}4,033 \\
4,944 \\
8,494 \\
6,148 \\
6,809 \\
5,874\end{array}$ \\
\hline
\end{tabular}




\section{TABLE 22.-Number of cattle registered by the American Guernsey Cattle Club, Jan. 1, 1900, to Dec. 31, 1921.}

[Secretary of the American Guernsey Cattle Club.]

\begin{tabular}{|c|c|c|c|c|c|c|c|}
\hline Year. & Bulls. & Cows. & Total. & Year. & Bulls. & Cows. & Total. \\
\hline 1900. & 608 & 896 & 1,504 & 1911.. & 2,402 & 4, 001 , & 6,403 \\
\hline 190 & 647 & 1,172 & 1,819 & 1912. & 2,942 & 4,578 & 7.520 \\
\hline 1902 & 726 & 1,267 & 1, 993 & 1913. & 3,653 & 5,642 & 9,295 \\
\hline 1903. & 746 & 1,289 & 2,035 & 1914. & 4,348 & 6,937 & 11,285 \\
\hline 1904. & 737 & 1,261 & 1,998 & 1915. & 4,765 & 6,535 & 11,300 \\
\hline 1905. & 847 & 1,612 & 2,459 & 1916. & 5,030 & 7,654 & 12,681 \\
\hline $1906 \ldots$ & 950 & 1,964 & 2,914 & $1917 \ldots$ & 6,167 & 9,366 & 15,533 \\
\hline $1907 \ldots$ & 1,118 & 1, 966 & 3,084 & $1918 \ldots$ & 6,108 & 9,356 & 15,464 \\
\hline 1908. - & 1,291 & 2,191 & 3,482 & $1919 \ldots$ & 7,648 & 11,781 & 19,429 \\
\hline $1909 .$. & 1,841 & 3,836 & 5,677 & $1920 \ldots$ & 7,427 & 11,956 & 19,383 \\
\hline $1910 . . .$. & 2,420 & 4, 194 & 6,614 & $1921 \ldots$. & 8,036 & 13,971 & 22,007 \\
\hline
\end{tabular}

The total number of animals in the herdbook of the American Guernsey Cattle Club to Jan. 1, 1921, is: Bulls, 68,804; cows, 112,003; total, 180,807 .

TABLE 23.-Number of cattle registered by the HolsteinFriesian Association of America for the years 1900 to 1921, inclusive.

[Secretary, Holstein-Friesian Association.]

\begin{tabular}{|c|c|c|c|c|c|c|c|}
\hline $\begin{array}{c}\text { Year } \\
\text { ended } \\
\text { Apr. } 30-\end{array}$ & Bulls. & Cows. & Total. & $\begin{array}{c}\text { Year } \\
\text { ended } \\
\text { Apr.30- }\end{array}$ & Bulls. & Cows. & Total. \\
\hline 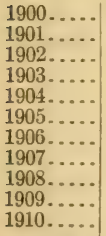 & $\begin{array}{l}1,365 \\
1,460 \\
1,738 \\
2,088 \\
2,477 \\
3,226 \\
3,842 \\
4,841 \\
5,684 \\
7,021 \\
9,689\end{array}$ & $\begin{array}{r}3,381 \\
3,648 \\
4,252 \\
4,753 \\
5,567 \\
6,547 \\
7,918 \\
9,809 \\
10,850 \\
12,570 \\
16,487\end{array}$ & $\begin{array}{r}4,746 \\
5,108 \\
5,990 \\
6,841 \\
8,044 \\
9,773 \\
11,760 \\
14,650 \\
16,534 \\
19,591 \\
26,176\end{array}$ & 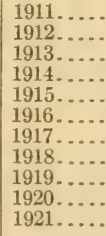 & $\begin{array}{l}12,472 \\
13,743 \\
16,364 \\
18,336 \\
25,617 \\
26,116 \\
24,749 \\
28,730 \\
30,298 \\
36,791 \\
39,585\end{array}$ & $\begin{array}{l}20,417 \\
23,792 \\
26,951 \\
29,750 \\
42,063 \\
46,549 \\
49,098 \\
59,549 \\
60,589 \\
77,712 \\
88,265\end{array}$ & $\begin{array}{r}32,889 \\
37,535 \\
43,315 \\
48,086 \\
67,680 \\
72,665 \\
73,847 \\
85,279 \\
90,887 \\
114,503 \\
127,850\end{array}$ \\
\hline
\end{tabular}

TABLE 24.-Number of cattle registered by the American Jersey Cattle Club for the years 1900 to 1921 .

[Secretary, American Jersey Cattle Club.]

\begin{tabular}{|c|c|c|c|c|c|c|c|}
\hline Year. & Bulls. & Cows. & Total. & Year. & Bulls. & Cows. & Total. \\
\hline & & & & & & & \\
\hline & & & & & & & \\
\hline & 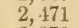 & & & & 9 & & \\
\hline & & & & & $10, \mathrm{C}$ & & \\
\hline & 2 & 7,4 & 9 & & 9 & & 32 , \\
\hline & & & & & 10, & 24 , & 35 , \\
\hline & & & & & 14, & & \\
\hline & 3,7 & & & & 8,9 & 25 , & 34,30 \\
\hline & & & & & 10, & & \\
\hline & 5,2 & & & & 11, & & \\
\hline & 6,3 & & & & 11,213 & 31,123 & 40 3 \\
\hline
\end{tabular}




\section{TABLE 25.-Number of COW-TESTING and BULL ASSOCIATIONS in the United States.}

[Dairy Division, Bureau of Animal Industry.]

\begin{tabular}{|c|c|c|c|c|c|}
\hline Year. & $\begin{array}{l}\text { Cow-test- } \\
\text { ing asso- } \\
\text { ciations. }\end{array}$ & $\begin{array}{l}\text { Bull asso- } \\
\text { ciations. }\end{array}$ & Year. & $\begin{array}{l}\text { Cow-test- } \\
\text { ing asso- } \\
\text { ciations. }\end{array}$ & $\begin{array}{l}\text { Bull asso- } \\
\text { ciations. }\end{array}$ \\
\hline $\begin{array}{l}1906 \ldots \ldots \\
1907 \ldots \ldots \\
1908 \ldots \ldots \\
1909 \ldots \ldots \\
1910 \ldots \ldots \\
1911 \ldots \ldots \\
1912 \ldots \ldots \\
1913 \ldots \ldots\end{array}$ & $\begin{array}{r}1 \\
4 \\
6 \\
25 \\
40 \\
61 \\
82 \\
100\end{array}$ & $\begin{array}{r}\text { None. } \\
\text { None. } \\
3 \\
8 \\
9 \\
11 \\
11 \\
12\end{array}$ & $\begin{array}{l}1914 \ldots \ldots \\
1915 \ldots \ldots \\
1916 \ldots \\
1917 \ldots \\
1918 \ldots \\
1919 \ldots \ldots \\
1920 \ldots \ldots \\
1921 \ldots \ldots\end{array}$ & $\begin{array}{l}163 \\
211 \\
346 \\
459 \\
353 \\
385 \\
468 \\
452\end{array}$ & $\begin{array}{r}14 \\
15 \\
24 \\
36 \\
44 \\
78 \\
123 \\
158\end{array}$ \\
\hline
\end{tabular}

Number of bulls per bull association, average in each of the three years $1918,1919,1920$, was 5 .

Number of cows per bull association, average for the 3-year period, 233.

Number of cows per block in bull associations, average for the 3-year period, 48. $3 \mathrm{~S}$.

Number of members per bull association, average for the 3-year period,

Number of herds per cow-testing association, average for the 3-year period, 26.2.

Number of cows per cow-testing association, average for the 3-year period, 448.

\section{TABLE 26.--Average of 120 sets of yearly records of 96 COW-TESTING ASSOCIATIONS in the United States for the years 1910 to 1920, inclusive.}

[Dairy Division, Bureau of Animal Industry.]

\begin{tabular}{|c|c|c|c|}
\hline & $\begin{array}{l}\text { Cows on } \\
\text { test } \\
4 \text { months } \\
\text { and not } \\
\text { more } \\
\text { than } 12 .\end{array}$ & . & $\begin{array}{l}\text { Cows on } \\
\text { test } \\
4 \text { months } \\
\text { and not } \\
\text { more } \\
\text { than } 12 .\end{array}$ \\
\hline $\begin{array}{l}\text { Number of cows......... } \\
\text { Number of cow-years } 1 . \\
\text { Pounds of milk.......... } \\
\text { Butterfat (per cent).... } \\
\text { Pounds of fat........... } \\
\text { Price of butterfat....... } \\
\text { Value of product...... }\end{array}$ & $\begin{array}{r}41,990 \\
37,362 \\
5,989 \\
4.12 \\
247 \\
\$ 0.46 \\
112.39\end{array}$ & $\begin{array}{l}\text { Cost of roughage......... } \\
\text { Cost of grain ........... } \\
\text { Total feed cost........ } \\
\text { Income over cost of feed } \\
\text { Returns for } \$ 1 \text { ex- } \\
\text { pended for feed....... }\end{array}$ & $\begin{array}{r}\$ 32.03 \\
21.03 \\
53.06 \\
59.33 \\
2.11\end{array}$ \\
\hline
\end{tabular}

${ }^{1} \mathrm{~A}$ cow-year may be the record of 1 cow on test 12 months or it may be made up of the records of 2 or more cows, the sum of whose testing periods equals 12 months.

The average yield of American farm cows from year to year is estimated at nearly 4,000 pounds of milk, or 160 pounds of butterfat per year. 


\section{TABLE 27.--Estımated number and capacity of SILOS in important dairy States for the year 1918.}

[Bureau of Markets and Crop Estimates.]

\begin{tabular}{|c|c|c|c|}
\hline State. & $\begin{array}{l}\text { Number } \\
\text { of silos. }\end{array}$ & $\begin{array}{l}\text { Average } \\
\text { capacity. }\end{array}$ & $\begin{array}{c}\text { Total } \\
\text { capacity. }\end{array}$ \\
\hline 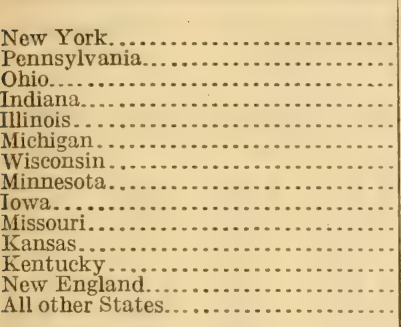 & $\begin{array}{l}55,000 \\
24,000 \\
25,000 \\
27,000 \\
30,000 \\
33,000 \\
55,000 \\
15,000 \\
16,000 \\
13,000 \\
11,000 \\
10,000 \\
35,000 \\
55,000\end{array}$ & $\begin{array}{r}\text { Tons. } \\
75 \\
65 \\
67 \\
70 \\
79 \\
70 \\
87 \\
95 \\
105 \\
90 \\
106 \\
80 \\
67 \\
77\end{array}$ & $\begin{array}{l}\text { ITons. } \\
4,125,000 \\
1,500,000 \\
1,675,000 \\
1,890,000 \\
2,370,000 \\
2,310,000 \\
4,785,000 \\
1,425,000 \\
1,680,000 \\
1,170,000 \\
1,166,000 \\
800,000 \\
2,345,000 \\
4,235,000\end{array}$ \\
\hline United States................... & 404,000 & 78 & $31,536,000$ \\
\hline
\end{tabular}

TABLE 28.- Total MILK PRODUCTION in the United States.

[Bureau of the Census.]

\begin{tabular}{|c|c|c|}
\hline Year. & $\begin{array}{c}\text { Milk } \\
\text { produetion. }\end{array}$ & Remarks. \\
\hline $\begin{array}{l}1869 \\
1879 \\
1889 \\
1899 \\
1909 \\
1919\end{array}$ & $\begin{array}{l}\text { Grallons. } \\
235,500,599 \\
530,129,755 \\
5,209,125,567 \\
7,728,583,349 \\
7,466,406,384 \\
7,805,143,792\end{array}$ & $\begin{array}{l}\text { Sold from farms. } \\
\text { Do. } \\
\text { Including milk from town cows. } \\
\text { Estimate by Bureau of the Census. } \\
\text { Do. }\end{array}$ \\
\hline
\end{tabular}

\section{TABLE 29.-Estimates of PRODUCTION and USES of} MILK in the United States.

[Dairy Division, Bureau of Animal Industry, and Bureau of Markets and Crop Estimates.]

[Million pounds $-000,000$ omitted.]

\begin{tabular}{|c|c|c|c|c|c|c|c|c|}
\hline \multirow[b]{2}{*}{ Product. } & \multicolumn{2}{|c|}{1917} & \multicolumn{2}{|c|}{1918} & \multicolumn{2}{|c|}{1919} & \multicolumn{2}{|c|}{1920} \\
\hline & Lbs. & $\begin{array}{c}\text { Per } \\
\text { cent. }\end{array}$ & Lbs. & $\begin{array}{c}\text { Per } \\
\text { cent. }\end{array}$ & Lbs. & $\begin{array}{c}\text { Per } \\
\text { cent. }\end{array}$ & Lbs. & $\begin{array}{l}\text { Per } \\
\text { cent. }\end{array}$ \\
\hline Total produc & 84,612 & 100 & 87,906 & 100 & 90,058 & 100 & 89,657 & 100 \\
\hline $\begin{array}{l}\text { Ice cream..... } \\
\text { Other products. } \\
\text { Household use. } \\
\text { Fed to calves... } \\
\text { Waste or loss... }\end{array}$ & \begin{tabular}{r}
34,664 \\
4,200 \\
2,438 \\
3,150 \\
\hdashline 36,500 \\
3,660
\end{tabular} \mid & $\begin{array}{r}41 \\
5 \\
2.9 \\
3.7 \\
43.1 \\
4.3 \\
\ldots . .\end{array}$ & $\begin{array}{r}16,800 \\
14,910 \\
4,000 \\
4,000 \\
3,300 \\
50 \\
38,900 \\
3,748 \\
2,198\end{array}$ & $\begin{array}{r}19.1 \\
16.9 \\
4.5 \\
4.5 \\
3.8 \\
.1 \\
44.3 \\
4.3 \\
2.5\end{array}$ & $\begin{array}{r}18,375 \\
14,385 \\
4,200 \\
4,813 \\
3,450 \\
216 \\
38,619 \\
3,500 \\
2,500\end{array}$ & $\begin{array}{r}20.4 \\
16.0 \\
4.7 \\
5.3 \\
3.8 \\
.2 \\
42.9 \\
3.9 \\
2.8\end{array}$ & $\begin{array}{r}18,135 \\
14,175 \\
3,624 \\
3,945 \\
3,575 \\
222 \\
39,090 \\
4,202 \\
2,689\end{array}$ & $\begin{array}{r}20.2 \\
15.8 \\
4.0 \\
4.4 \\
4.0 \\
0.2 \\
43.6 \\
4.7 \\
3.0\end{array}$ \\
\hline
\end{tabular}


TABLE 30.-Number and breeds of ACCREDITED and ONCE-TESTED CATTLE, showing cattle free from tuberculosis, as indicated by tuberculin test, up to June 30, 1920.

[U. S. Department of Agriculture; Circular 142 (YB.).]

\begin{tabular}{|c|c|c|c|c|}
\hline \multirow{2}{*}{ Breed. } & \multicolumn{2}{|c|}{$\begin{array}{l}\text { Once-tested with- } \\
\text { out reacting. }\end{array}$} & \multicolumn{2}{|c|}{$\begin{array}{l}\text { In accredited } \\
\text { herds. }\end{array}$} \\
\hline & Purebred. & Grade. & Purebred. & Grade. \\
\hline 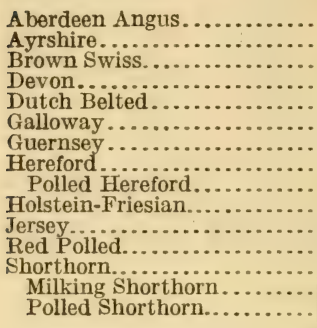 & $\begin{array}{r}3,286 \\
2,315 \\
686 \\
82 \\
48 \\
123 \\
9,049 \\
13,014 \\
1,120 \\
25,916 \\
18,045 \\
1,675 \\
20,249 \\
162 \\
368\end{array}$ & $\begin{array}{r}2,095 \\
2,137 \\
350 \\
25 \\
271 \\
143 \\
15,538 \\
6,277 \\
197 \\
45,859 \\
56,160 \\
1,265 \\
29,601 \\
287 \\
234\end{array}$ & $\begin{array}{r}2,072 \\
1,291 \\
387 \\
34 \\
18 \\
38 \\
6,651 \\
5,117 \\
777 \\
11,141 \\
10,669 \\
1,189 \\
7,606 \\
11 \\
320\end{array}$ & $\begin{array}{r}594 \\
274 \\
63 \\
6 \\
8 \\
40 \\
6,003 \\
1,816 \\
122 \\
12,599 \\
9,525 \\
155 \\
3,237 \\
15 \\
58\end{array}$ \\
\hline Total................ & 97,138 & 160,439 & 47,021 & 34,515 \\
\hline
\end{tabular}

A plan was adopted in 1917 by the Federal and State authorities to eradicate tuberculosis. This plan provides for "accredited herds."

An accredited herd is one which has been tuberculin-tested by the subcutaneous method, or any other test approved by the Bureau of Animal Industry, under the supervision of the Bureau of Animal Industry, or of a regularly employed veterinary inspector of a state in which cooperative tuberculosis-eradication work is conducted by the United States Department of Agriculture and the State. Further, it shall be a herd in which no animal affected with tuberculosis has been found upon two annual or three semiannual tuberculin tests, as described above, and by physical examination.

On June 30, 1921, there were in the United States 8,201 accredited herds and 49,814 once-tested free herds.

\section{TABLE 31.-PRICE of MILK in Chicago, net to farmer.}

[Milk News.]

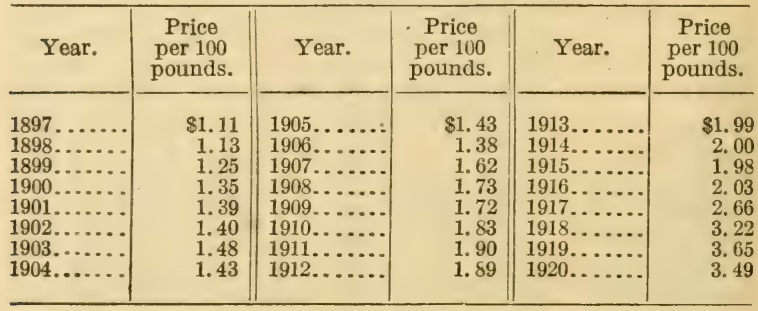




\section{TABLE 32.-Total annual RECEIPTS of MARKET MILK in New York City, Philadelphia, and Boston.}

[Milk Reporter.]

\begin{tabular}{|c|c|c|c|}
\hline Yea & $\begin{array}{l}\text { New York } \\
\text { City. }\end{array}$ & $\begin{array}{l}\text { Phila- } \\
\text { delphia. }\end{array}$ & Boston. \\
\hline $\begin{array}{l}\cdots \\
\cdots \cdots . . . \\
\cdots \cdots . . . \\
\cdots \ldots . .\end{array}$ & $\begin{array}{c}\text { Gallons. } \\
85,910,050 \\
92,202,650 \\
115,022,450 \\
158,994,000 \\
175,021,150 \\
179,966,900 \\
178,897,450 \\
187,091,700 \\
200,049,100 \\
212,170,850 \\
217,634,900 \\
226,675,950 \\
229,785,750 \\
240,144,450 \\
243,929,500 \\
246,597,650 \\
260,547,370 \\
279,686,190 \\
307,431,106 \\
326,316,250\end{array}$ & 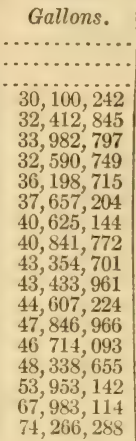 & $\begin{array}{l}28,558,491 \\
27,470,547 \\
25,957,819 \\
27,020,734 \\
25,151,590 \\
22,523,193 \\
26,004,808 \\
23,826,712 \\
25,909,556 \\
27,376,987 \\
29,629,053 \\
35,180,444 \\
36,578,654 \\
39,177,071 \\
41,595,081 \\
42,348,423\end{array}$ \\
\hline
\end{tabular}

The receipts at New York were in the form of whole milk, cream, and condensed milk.

In Boston the receipts were by railroad. Means of receipt in the other cities not stated. The totals for Boston from 1906 to 1916, inclusive, are from Massachusetts Public Document No.60 (1917), and from 1917 to 1919, from E. M. Hartwell, statistician, Boston.

\section{TABLE 33.-Prevailing New York price for Grade B milk, net to farmer.}

[Milk Reporter.]

\begin{tabular}{|c|c|c|c|c|c|}
\hline Year. & $\begin{array}{l}\text { Average } \\
\text { price per } \\
\text { quart. }\end{array}$ & Year. & $\begin{array}{c}\text { Average } \\
\text { price per } \\
\text { quart. }\end{array}$ & Year. & $\begin{array}{c}\text { Average } \\
\text { price per } \\
\text { quart. }\end{array}$ \\
\hline $\begin{array}{l}8751 \ldots \\
876 \ldots \\
877 \ldots \ldots \\
8781 \ldots \\
879 \ldots \\
880 \ldots \\
881 \ldots \\
882 \ldots \\
883 \ldots \\
884 \ldots \\
885 \\
886 \\
887 \ldots\end{array}$ & $\begin{array}{r}\text { Cents. } \\
3.58 \\
3.37 \\
3.27 \\
2.60 \\
2.33 \\
2.88 \\
2.94 \\
3.25 \\
3.26 \\
3.00 \\
2.79 \\
2.80 \\
2.81 \\
2.83 \\
2.59 \\
2.63\end{array}$ & $\begin{array}{l}1891 \ldots \\
1892 \ldots \\
1893 \ldots \\
1891 \ldots \\
1895 \ldots \\
1896 \ldots \\
1897 \ldots \\
1898 \ldots \\
1899 \ldots \\
1900 \ldots \\
1901 \ldots \\
1902 \ldots \\
1903 \ldots \\
1904 \ldots \\
1905 \ldots\end{array}$ & $\begin{array}{r}\text { Cents. } \\
2.66 \\
2.68 \\
2.79 \\
2.63 \\
2.52 \\
2.34 \\
2.35 \\
2.38 \\
2.53 \\
2.74 \\
2.62 \\
2.88 \\
2.88 \\
2.75 \\
2.89 \\
3.01\end{array}$ & $\begin{array}{l}1907 \ldots \ldots \\
1908 \ldots \ldots \\
1909 \ldots \\
1910 \ldots \ldots \\
1911 \ldots \ldots \\
1912 \ldots \\
1913 \ldots \ldots \\
1914 \ldots \ldots \\
1915 \ldots \ldots \\
1916 \ldots \ldots \\
1917 \ldots \ldots \\
1918 \ldots \ldots \\
1919 \ldots \ldots \\
1920 \ldots \ldots \\
1921 \ldots \ldots\end{array}$ & $\begin{array}{r}\text { Cents. } \\
3.35 \\
3.29 \\
3.38 \\
3.68 \\
3.36 \\
3.68 \\
3.53 \\
3.50 \\
3.50 \\
3.88 \\
5.68 \\
7.00 \\
7.58 \\
7.57 \\
6.42\end{array}$ \\
\hline
\end{tabular}

1 Price in gold during period of inflated currency: 1875, 3.11 cents; $1876,3.02$ cents; $1877,3.12$ cents; $1878,2.58$ cents. 


\section{TABLE 34.-Unit requirements for producing 100 pounds of milk in different sections of the United States.}

[Dairy Division, Bureau of Animal Industry.]

\begin{tabular}{|c|c|c|c|c|c|c|}
\hline Item. & \multicolumn{2}{|c|}{ Indiana. ${ }^{1}$} & \multicolumn{2}{|c|}{ Vermont. ${ }^{2}$} & \multicolumn{2}{|c|}{ North Carolina. ${ }^{8}$} \\
\hline \multirow[t]{2}{*}{$\begin{array}{l}\text { Average yearly milk } \\
\text { production per } \\
\text { cow......pounds.. } \\
\text { Per cent butterfat... }\end{array}$} & \multicolumn{2}{|c|}{$\begin{array}{r}6,937 \\
3.8\end{array}$} & \multicolumn{2}{|c|}{$\begin{array}{r}5,252 \\
3.9\end{array}$} & \multicolumn{2}{|c|}{4,915} \\
\hline & Winter. & $\begin{array}{l}\text { Sum- } \\
\text { mer. }\end{array}$ & Winter. & $\begin{array}{l}\text { Sum- } \\
\text { mer. }\end{array}$ & Winter. & $\begin{array}{l}\text { Sum- } \\
\text { mer. }\end{array}$ \\
\hline $\begin{array}{l}\text { Grain......pounds.. } \\
\text { Dry roughage.do.... } \\
\text { Succulent roughage }\end{array}$ & $\begin{array}{l}35.6 \\
66.8\end{array}$ & $\begin{array}{l}20.0 \\
27.4\end{array}$ & $\begin{array}{r}33.1 \\
129.9\end{array}$ & $\begin{array}{r}8.7 \\
18.7\end{array}$ & $\begin{array}{l}57.8 \\
78.5\end{array}$ & $\begin{array}{l}48.4 \\
36.9\end{array}$ \\
\hline $\begin{array}{l}\text { Haiuling and grind-. } \\
\text { ing concentrates... }\end{array}$ & 147.6 & 60.1 & 191.3 & 27.8 & 181.5 & 87.0 \\
\hline $\begin{array}{l}\text { Bedding ...pounds.. } \\
\text { Pasture.......acres.. }\end{array}$ & 20.3 & $\$ 0.14$ & $\begin{array}{r}\$ 0.02 \\
11.2\end{array}$ & $\begin{array}{l}\$ 0.005 \\
\cdots \cdots\end{array}$ & $\$ 0.008$ & \\
\hline $\begin{array}{l}\text { Human labor } \\
\text { Horse labor.... hours... } \\
\text { Other costs......... }\end{array}$ & $\begin{array}{r}2.5 \\
.3 \\
\$ 0.3 \times 5 \\
\end{array}$ & $\begin{array}{r}2.2 \\
\$ 0.2 \\
\$ 0.393 \\
\end{array}$ & $\begin{array}{r}2.7 \\
\$ 0.555 \\
\end{array}$ & $\begin{array}{r}2.0 \\
\$ 0.4 \\
\$\end{array}$ & $\begin{array}{r}3.6 \\
51.8 \\
\$ 0.537 \\
\end{array}$ & $\begin{array}{r}3.2 \\
51.8 \\
\$ 0.495 \\
\end{array}$ \\
\hline $\begin{array}{c}\text { Credit for calves } \\
\text { Credit for manure } \\
\ldots . . . . . . \text { pounds.. }\end{array}$ & .012 & .013 & .025 & .009 & $\$ 0.548$ & $\$ 0.430$ \\
\hline Item. & Washir & ngton. ${ }^{4}$ & Louis & iana. 6 & Neb & ska.7 \\
\hline \multirow[t]{2}{*}{$\begin{array}{l}\text { Average yearly milk } \\
\text { production per } \\
\text { cow......pounds.. } \\
\text { Per cent butterfat... }\end{array}$} & \multicolumn{2}{|c|}{$\begin{array}{r}7,833 \\
3.7\end{array}$} & \multicolumn{2}{|c|}{$\begin{array}{r}3,106 \\
4.4\end{array}$} & \multicolumn{2}{|c|}{$\begin{array}{l}5,823 \\
3.65\end{array}$} \\
\hline & Winter. & $\begin{array}{l}\text { Sum- } \\
\text { mer. }\end{array}$ & Winter. & $\begin{array}{l}\text { Sum- } \\
\text { mer. }\end{array}$ & Winter. & $\begin{array}{l}\text { Sum- } \\
\text { mer. }\end{array}$ \\
\hline $\begin{array}{l}\text { Grain.....pounds.. } \\
\text { Dry roughage.do.... } \\
\text { Succulent roughage }\end{array}$ & $\begin{array}{l}29.4 \\
92.8\end{array}$ & $\begin{array}{l}5.2 \\
7.5\end{array}$ & $\begin{array}{l}72.4 \\
38.3\end{array}$ & $\begin{array}{r}52.5 \\
1.9\end{array}$ & $\begin{array}{l}41.2 \\
95.3\end{array}$ & $\begin{array}{l}11.0 \\
51.2\end{array}$ \\
\hline Hauling and grind- & 143.3 & 40.4 & 78.4 & 8.1 & 93.6 & 29.3 \\
\hline & $\begin{array}{r}\$ 0.022 \\
9.0\end{array}$ & $\begin{array}{r}\$ 0.003 \\
.1\end{array}$ & $\$ 0.095$ & $\$ 0.050$ & $\begin{array}{r}\$ 0.016 \\
11.1\end{array}$ & $\begin{array}{r}\$ 0.004 \\
.5\end{array}$ \\
\hline $\begin{array}{l}\text { Pasture......acres.. } \\
\text { Human labor }\end{array}$ & & .025 & $\$ 0.155$ & $\$ 0.197$ & $\$ 0.108$ & $\$ 0.653$ \\
\hline $\begin{array}{l}\text { Horse labor.... do..... } \\
\text { Other costs......... }\end{array}$ & $\begin{array}{r}1.9 \\
\$ 01 \\
\$ 0.576 \\
\end{array}$ & $\begin{array}{r}1.3 \\
.015 \\
\$ 0.406 \\
\end{array}$ & $\begin{array}{r}5.8 \\
.9 \\
\$ 1.220 \\
\end{array}$ & $\begin{array}{r}5.0 \\
.7 \\
\$ 0.803 \\
\end{array}$ & $\begin{array}{r}2.0 \\
.06 \\
\$ 0.869 \\
\end{array}$ & $\begin{array}{r}1.9 \\
.08 \\
\$ 0.889 \\
\end{array}$ \\
\hline $\begin{array}{c}\text { Credit for calves } \\
. . . . . \text { part of calf.. }\end{array}$ & 0.017 & 0.009 & .033 & .023 & .02 & .01 \\
\hline $\begin{array}{c}\text { Credit for manure } \\
. . . . . . . . . p o u n d s . .\end{array}$ & 115 & 13 & 128 & 78 & 244 & 20 \\
\hline
\end{tabular}

1 U. S. Dept. of Agric. Bul. 858. 2 U. S. Dept. of Agric. Bul. 923. 3 U. S. Dept. of Agric. Bul. 266.

1 U. S. Dept. of Agric. Bul. 919.
5 Farmers retailed own milk. ${ }^{6} 6$ U. S. Dept. of Agric. Bul. 955. 7 U.S. Dept. of Agric. Bul. 972. 


\section{TABLE 35.-Produrction of DAIRY PRODUCTS in the United States for the year 1919, by months.}

[Bureau of Markets and Crop Estimates.]

\begin{tabular}{|c|c|c|c|c|c|c|}
\hline \multirow{2}{*}{ Month. } & \multirow{2}{*}{$\begin{array}{c}\text { Creamery } \\
\text { butter. }\end{array}$} & \multirow{2}{*}{$\begin{array}{c}\text { Whey } \\
\text { butter. }\end{array}$} & \multicolumn{3}{|c|}{ American Cheddar cheese. } & \multirow{2}{*}{$\begin{array}{c}\text { Swiss } \\
\text { cheese } \\
\text { (includ- } \\
\text { ing } \\
\text { block). }\end{array}$} \\
\hline & & & $\begin{array}{l}\text { Whole } \\
\text { milk. }\end{array}$ & $\begin{array}{l}\text { Part } \\
\text { skim. }\end{array}$ & $\begin{array}{l}\text { Full } \\
\text { skim. }\end{array}$ & \\
\hline $\begin{array}{c}1919 . \\
\text { Jan...... } \\
\text { Feb...... } \\
\text { Mar..... } \\
\text { Apr...... } \\
\text { May...... } \\
\text { June..... } \\
\text { July...... } \\
\text { Aug..... } \\
\text { Sept...... } \\
\text { Oct...... } \\
\text { Nov...... } \\
\text { Dec..... }\end{array}$ & \begin{tabular}{|r} 
Pounds. \\
$52,198,957$ \\
$44,342,568$ \\
$54,822,396$ \\
$67,487,317$ \\
$103,941,021$ \\
$119,357,493$ \\
$104,751,266$ \\
$84,840,310$ \\
$69,102,578$ \\
$58,722,557$ \\
$45,040,656$ \\
$46,662,021$
\end{tabular} & \begin{tabular}{|r|} 
Pounds. \\
372,478 \\
225,545 \\
276,892 \\
383,485 \\
596,694 \\
717,123 \\
694,382 \\
657,863 \\
597,292 \\
424,017 \\
430,863 \\
220,674
\end{tabular} & \begin{tabular}{|c|} 
Pounds. \\
$10,955,738$ \\
$11,854,735$ \\
$19,008,970$ \\
$21,641,729$ \\
$34,849,069$ \\
$44,599,120$ \\
$35,465,017$ \\
$30,940,232$ \\
$26,256,549$ \\
$23,114,457$ \\
$13,106,756$ \\
$10,043,643$
\end{tabular} & \begin{tabular}{|c|} 
Pounds. \\
610,541 \\
238,703 \\
495,475 \\
734,953 \\
900,810 \\
942,233 \\
732,809 \\
528,176 \\
276,674 \\
248,049 \\
230,276 \\
250,493
\end{tabular} & \begin{tabular}{|r|} 
Pounds. \\
423,537 \\
430,574 \\
326,297 \\
588,741 \\
874,757 \\
$1,448,147$ \\
821,415 \\
710,736 \\
528,067 \\
378,272 \\
249,299 \\
205,514
\end{tabular} & $\begin{array}{r}\text { Pounds. } \\
131,949 \\
172,335 \\
269,235 \\
597,791 \\
2,850,662 \\
3,910,881 \\
3,440,549 \\
3,227,488 \\
2,629,450 \\
1,860,081 \\
848,190 \\
448,689\end{array}$ \\
\hline Total.. & 851,26 & & \multicolumn{2}{|l|}{$281,836,015$} & & \\
\hline Month. & $\begin{array}{c}\text { Brick } \\
\text { and } \\
\text { Münster } \\
\text { cheese. }\end{array}$ & $\begin{array}{l}\text { Lim- } \\
\text { burger } \\
\text { cheese. }\end{array}$ & $\begin{array}{l}\text { Cottage, } \\
\text { pot, and } \\
\text { baker's } \\
\text { cheese. }\end{array}$ & $\begin{array}{l}\text { Cream } \\
\text { and } \\
\text { Neuf- } \\
\text { châtel } \\
\text { cheese. }\end{array}$ & $\begin{array}{c}\text { Italian } \\
\text { varieties } \\
\text { of } \\
\text { cheese. }\end{array}$ & $\begin{array}{c}\text { All } \\
\text { other } \\
\text { varieties } \\
\text { of } \\
\text { cheese. }\end{array}$ \\
\hline 1919. & $\begin{array}{r}\text { Pounds. } \\
2,636,940 \\
2,408,269 \\
2,668,920 \\
3,146,302 \\
4,039,841 \\
4,334,932 \\
2,872,007 \\
2,552,768 \\
2,462,945 \\
3,794,110 \\
3,523,507 \\
3,080,463\end{array}$ & $\begin{array}{r}\text { Pounds. } \\
177,294 \\
210,818 \\
237,678 \\
502,245 \\
1,220,499 \\
1,301,585 \\
886,299 \\
874,133 \\
748,086 \\
703,939 \\
473,155 \\
289,810\end{array}$ & $\begin{array}{r}\text { Pounds. } \\
2,109,591 \\
2,443,069 \\
2,707,964 \\
3,701,192 \\
3,214,328 \\
2,925,902 \\
2,344,858 \\
2,201,372 \\
1,895,083 \\
2,180,766 \\
1,855,898 \\
2,205,306\end{array}$ & $\begin{array}{c}\text { Pounds. } \\
450,177 \\
413,916 \\
474,339 \\
430,193 \\
440,168 \\
445,583 \\
506,491 \\
402,011 \\
376,972 \\
423,218 \\
507,611 \\
629,960\end{array}$ & $\begin{array}{r}\text { Pounds. } \\
317,446 \\
298,693 \\
335,177 \\
358,339 \\
457,398 \\
451,674 \\
334,639 \\
312,054 \\
279,230 \\
329,434 \\
256,158 \\
257,119\end{array}$ & \begin{tabular}{|r} 
Pounds. \\
513,326 \\
546,372 \\
769,412 \\
863,582 \\
892,360 \\
784,094 \\
661,120 \\
627,851 \\
703,497 \\
$1,264,474$ \\
985,499 \\
$1,049,910$
\end{tabular} \\
\hline Total. & $37,521,004$ & $7,625,541$ & $29,785,329$ & $5,500,639 \mid 3$ & $3,987,361$ & $9,661,497$ \\
\hline
\end{tabular}

Note.-This table is not quite complete, as a few reports were received after it was made. For final total butter figures see Table 40; for total cheese made see Table 51; and for condensed and evaporated milk see Table 61.

The figures in Tables 35 and 36 are taken from the Market Reporter (published by the Bureau of Markets and Crop Estimates), Apr. 3, 1920. They include only such factories as reported for both 1918 and 1919, and represent about $9: 3$ per cent of the total number of factories.

See Table 39 for totalcreamery butter; and Table 40 for total creamery and whey butter. 


\section{TABLE 36.-Production of CONDENSED and EVAPO- RATED MILK in the United States for the year 1919, by months.}

[Bureau of Markets and Crop Estimates.]

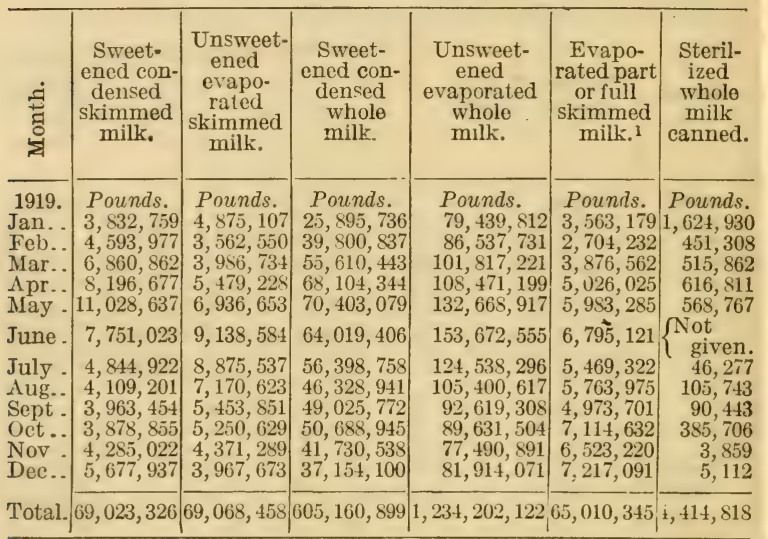

1 Modified with foreign fats.

The figures in Tables 35 and 36 are taken from the Market Reporter (published by the Bureau of Markets and Crop Estimates), A pr. 3, 1920. They include only such factories as reported for both 1918 and 1919, and represent about 93 per cent of the total number of factories.

For total condensed and evaporated milk, see Table 61 .

\section{TABLE 37.-PRODUCTION and EXPORTATION of RENOVATED BUTTER, by years.}

[Bureau of Internal Revenue and Dairy Division, Bureau of Animal Industry.]

\begin{tabular}{|c|c|c|c|c|c|c|c|}
\hline $\begin{array}{l}\text { Year. } \\
\text { ended } \\
\text { June } \\
30-\end{array}$ & $\mid \begin{array}{c}\text { Fac- } \\
\text { to- } \\
\text { ries.1 }\end{array}$ & $\begin{array}{l}\text { Produc- } \\
\text { tion. }\end{array}$ & Exports. & $\begin{array}{c}\text { Year } \\
\text { ended } \\
\text { June } \\
30 .\end{array}$ & $\begin{array}{c}\text { Fac- } \\
\text { to- } \\
\text { ries.1 }\end{array}$ & $\begin{array}{c}\text { Produc- } \\
\text { tion. }\end{array}$ & Exports. \\
\hline 102 & 55 & $\begin{array}{l}\text { Pounds. } \\
\text { No record. }\end{array}$ & $\begin{array}{l}\text { Pounds. } \\
\text { No record }\end{array}$ & 1912 & 39 & $\begin{array}{l}\text { Pounds. } \\
46.387 .398\end{array}$ & Pounds. \\
\hline & 81 & & & & 39 & $38,354,762$ & \\
\hline & 77 & & & 191 & 34 & & \\
\hline & 78 & 60,0 & 3,8 & 191 & 29 & & 1,91 \\
\hline & 64 & & & & 28 & & 133 \\
\hline & 51 & & & & 22 & & \\
\hline & 49 & & & 191 & 19 & & \\
\hline & 49 & & 1,11 & & 15 & & \\
\hline & 42 & & & & 11 & & \\
\hline & .45 & $39,292,5$ & 118,890 & 1921 & 11 & & 4,0 \\
\hline
\end{tabular}

1 Total number of factories at end of fiscal year.

Production is taken from reports of the Bureau of Internal Revenue; and exportsare taken from the reports of inspectors of the Dairy Division, Bureau of Animal Industry, Department of Agriculture. 
TABLE 38.-DAIRY PRODUCTS made during 1919 in FACTORIES in the United States, by States.

[Bureau of Markets and Crop Estimates.]

\begin{tabular}{|c|c|c|c|}
\hline State. & $\begin{array}{l}\text { Creamery } \\
\text { butter. }{ }^{1}\end{array}$ & $\begin{array}{l}\text { Factory } \\
\text { cheese. }^{2}\end{array}$ & $\begin{array}{l}\text { Condensed } \\
\text { and evapo- } \\
\text { rated milk. }\end{array}$ \\
\hline Alabama & Pounds. & Pounds. & Pounds. \\
\hline Arizona. . . . . . . & $1,000,118$ & 463,717 & $\begin{array}{r}1,129,304 \\
16,363,677\end{array}$ \\
\hline Arkansas... & 363,384 & & $10,000,016$ \\
\hline California...... & $61,795,295$ & $8,940,302$ & $78,636,340$ \\
\hline Colorado................... & $13,144,464$ & 507,696 & $16,903,909$ \\
\hline Connecticut... & 929,551 & 111 & 26,559 \\
\hline Delaware............................. & 253,286 & 200,237 & ........ \\
\hline District of Columbia........ & 5,375 & & \\
\hline Florida..................... & 16,755 & $\ldots .$. & 76,138 \\
\hline 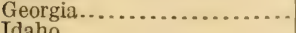 & 315,802 & $\ddot{2} 382428$ & \\
\hline Idaho.......... & $4,514,263$ & $2,582,428$ & $11,093,091$ \\
\hline Mlin & $44,621,358$ & $6,294,871$ & $188,950,296$ \\
\hline$\ldots \ldots \ldots \ldots$ & $44,658,534$ & 185,230 & $23,662,666$ \\
\hline Iow: & $87,914,633$ & 951,425 & $6,653,397$ \\
\hline Kan & $35,642,291$ & 25,498 & $19,834,406$ \\
\hline Kentuck & $5,321,193$ & ...... & $\cdots$ \\
\hline $\begin{array}{l}\text { Louisiana..... } \\
\text { Maine......... }\end{array}$ & $\begin{array}{r}46,409 \\
1,140,850\end{array}$ & 1,100 & \\
\hline Maryland....... & 315,305 & 237,926 & $\begin{array}{l}6,894,304 \\
9,111,194\end{array}$ \\
\hline Massachusetts. & $2,849,270$ & 106,675 & $2,215,243$ \\
\hline Michigan....... & $45,206,770$ & $7,690,485$ & $198,651,629$ \\
\hline Minnesota... & $130,785,598$ & $9,766,750$ & $2,360,121$ \\
\hline Mississippi . . . & $2,476,787$ & . & \\
\hline Missouri..... & $38,411,403$ & 304,983 & 39,831 \\
\hline Montana.... & $5,389,032$ & 318,398 & \\
\hline Nebraska..... & $60,467,056$ & 39,343 & $3,071,270$ \\
\hline Nevada.......... & $1,725,494$ & 0 & \\
\hline New Hampshire... & 397,198 & 258,843 & 76,332 \\
\hline New Jersey......... & 178,796 & 856,195 & $10,571,144$ \\
\hline $\begin{array}{l}\text { New Mexico.............. } \\
\text { New York............... }\end{array}$ & $\begin{array}{r}5,673 \\
13,715,704\end{array}$ & 452 & $\cdots$ \\
\hline 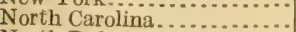 & 10, 829,286 & $\begin{array}{r}08,005,702 \\
228,765\end{array}$ & ........... \\
\hline North Dakota........... & $14,696,566$ & & \\
\hline Ohio ........ & $60,573,159$ & $4,546,836$ & $123,442,732$ \\
\hline Oklahoma.. & $10,481,270$ & 7,550 & \\
\hline Oregon....... & $14,431,827$ & $8,465,306$ & $33,947,234$ \\
\hline Pennsylvania.. & $12,445,578$ & $6,307,821$ & $181,897,826$ \\
\hline Rhode Island.. & 65,392 & 4,294 & 211 \\
\hline South Carolina. & 26,580 & 348 & $\ldots$ \\
\hline South Dakota. & $17,478,570$ & 56,348 & \\
\hline Tennessee... & $3,735,157$ & 51,033 & 960 \\
\hline Texas....... & $8,288,644$ & 17,603 & 11,644 \\
\hline & $3,796,394$ & 914,605 & $20,479,439$ \\
\hline Vermont... & $10,676,538$ & $3,581,569$ & $38,997,068$ \\
\hline Virginia...... & $1,597,340$ & 59,991 & $5,221,714$ \\
\hline Washington... & $18,486,591$ & $1,257,254$ & $129,482,526$ \\
\hline West Virginia......... & 328,160 & 55,750 & 400,122 \\
\hline Wisconsin . ............ & $85,054,334$ & $263,481,352$ & $457,214,442$ \\
\hline Wyoming ..... & $1,139,556$ & $1,612,356$ & ............. \\
\hline Total.. & $868,124,806$ & $399,239,446$ & $2,030,957,648$ \\
\hline
\end{tabular}

1 See Tables 34,35 and 40 for whey butter.

2 Cottage, pot, and baker's cheese are not included under cheese.

3 Sterilized canned milk, condensed buttermilk, and milk modified with foreign fats are not included in this table. Production of sterilized canned milk for 1919 was $4,420,713$ pounds.

Note.-A few reports were made after this table was made; see Tables 40,51 , and 61 for final totals. 
TABLE 39.-DAIRY PRODUCTS made during 1920 in FACTORIES in the United States, by States.

[Bureau of Markets and Crop Estimates.]

\begin{tabular}{|c|c|c|c|}
\hline Stat & $\begin{array}{l}\text { Creamery } \\
\text { butter. }\end{array}$ & $\begin{array}{c}\text { Factory } 1 \\
\text { cheese. }\end{array}$ & $\begin{array}{l}\text { Condensed } \\
\text { and evapo- } \\
\text { rated milk. }\end{array}$ \\
\hline labama. & $\begin{array}{l}\text { Pounds. } \\
398,000\end{array}$ & $\begin{array}{l}\text { Pounds. } \\
\text {............. }\end{array}$ & $\begin{array}{l}\text { Pounds. } \\
223,000\end{array}$ \\
\hline & & & \\
\hline & 828, & 227,000 & 3,452 , \\
\hline & 1,870, & 8,518 & 83, \\
\hline onnecti & 12, & 531,000 & 15,7 \\
\hline onnecti & $\begin{array}{l}87,000 \\
350,000\end{array}$ & 147,000 & \\
\hline $\mathrm{CO}$ & 503,0 & & $\cdot$ \\
\hline & 7000 & & \\
\hline & $4,660,000$ & 8000 & 2,000 \\
\hline & 41, 051, & 3,96 & 7,000 \\
\hline & & & 07,000 \\
\hline & & & $5,637,000$ \\
\hline & & & $19,004,000$ \\
\hline & 7,8 & & 240,000 \\
\hline & & & \\
\hline & & 00 & 000 \\
\hline etits & & & $2,383,000$ \\
\hline & & 6,56 & $160,236,000$ \\
\hline & 120 , & $5,841,000$ & $1,884,000$ \\
\hline & & & \\
\hline & & & \\
\hline & $\begin{array}{r}5, \\
56\end{array}$ & $\begin{array}{r}295,000 \\
3,000\end{array}$ & 182,000 \\
\hline & 2,0 & & 102,000 \\
\hline & & $700,000^{\circ}$ & \\
\hline & $\begin{array}{r}143, \\
6\end{array}$ & 693,000 & $4,819,000$ \\
\hline & $\begin{array}{r}0,000 \\
16,949,000\end{array}$ & 53,21 & $288,390,000$ \\
\hline & 0 & 109,000 & $200,000,000$ \\
\hline & & & \\
\hline & & 2,95 & $113,231,000$ \\
\hline & $\begin{array}{r}3,596,000 \\
14,288,000\end{array}$ & 8,48 & $31,763,000$ \\
\hline & $11,422,000$ & $6,245,000$ & $140,002,000$ \\
\hline & & ............ & …............ \\
\hline & & & \\
\hline & 14, 5 , & & . \\
\hline & $\begin{array}{l}5,9 \\
9 ; 1\end{array}$ & & 246,00 \\
\hline & 3,56 & 855 & $16,943,000$ \\
\hline & $13,253,000$ & 1,850, & $26,010,00$ \\
\hline & $2,210,000$ & 35 & $5,651,00$ \\
\hline & $23,751,0$ & 1, 463, & $83,690,00$ \\
\hline & & & 889,00 \\
\hline $\begin{array}{l}\text { Wiscons } \\
\text { Wyomin }\end{array}$ & $\begin{array}{r}97,355,000 \\
875,000\end{array}$ & $\begin{array}{r}255,286,000 \\
1,203,000\end{array}$ & $410,195,00$ \\
\hline Total. & $863,577,000$ & $362,431,000$ & $1,583,638,000$ \\
\hline
\end{tabular}

${ }^{1}$ Cheese does not include either cottage, baker's, or pot cheese.

2 Condensed and evaporated milk does not include condensed buttermilk or milk modified with foreign fats; but does include sterilized canned milk. 


\section{5}

\section{TABLE 40 .- Total PRODUCTION of farm and factory BUT'TER in the United States.}

[Census Bureau and Bureau of Markets and Crop Estimates.] [ $\mathrm{c}=$ census. $\mathrm{m}=$ markets.]

\begin{tabular}{|c|c|c|c|}
\hline Year. & $\begin{array}{c}\text { Creamery } \\
\text { and whey } \\
\text { butter. }\end{array}$ & $\begin{array}{l}\text { Farm-made } \\
\text { butter. }\end{array}$ & Total butter. \\
\hline 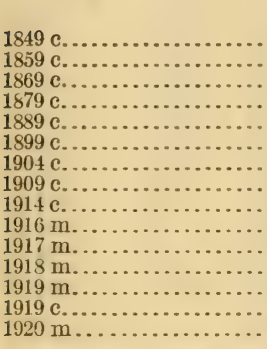 & 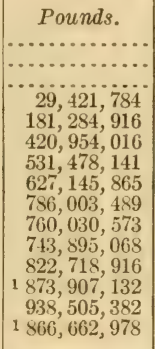 & 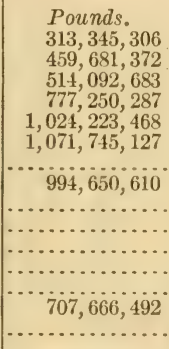 & $\begin{array}{r}\text { Pounds. } \\
313,345,306 \\
459,681,372 \\
514,092,68 \\
806,672,071 \\
1,205,508,38 \\
1,492,699,143 \\
\ldots 1,621,796,475 \\
\ldots \ldots \ldots \\
\ldots \ldots \ldots . . .\end{array}$ \\
\hline
\end{tabular}

1 Whey butter included in Bureau of Markets reports for 1918, 1919, and 1920 . Production of whey butter in $1918,5,510,213 ; 1919,5,597,308$; $1920,3,085,978$.

\section{TABLE 41.-Total number of CREAMERIES in the United States.}

[Dairy Division, Bureau of Animal Industry.]

\begin{tabular}{|c|c|c|c|c|c|}
\hline Year. & $\begin{array}{l}\text { Number } \\
\text { of cream- } \\
\text { eries. }\end{array}$ & Year. & $\begin{array}{l}\text { Number } \\
\text { of cream- } \\
\text { eries. }\end{array}$ & Year. & $\begin{array}{l}\text { Number } \\
\text { of cream- } \\
\text { eries. }\end{array}$ \\
\hline $\begin{array}{l}1908 \ldots \ldots \\
1909 \ldots \ldots \\
1910 \ldots \ldots \\
1911 \ldots \ldots \\
1912 \ldots \ldots\end{array}$ & $\begin{array}{l}5,431 \\
5,123 \\
5,030 \\
4,838 \\
4,751\end{array}$ & $\begin{array}{l}1913 . \ldots \ldots \\
1914 \ldots \ldots \\
1915 . \ldots \ldots \\
1916 \ldots \ldots \\
1917 \ldots \ldots\end{array}$ & $\begin{array}{r}4,859 \\
4,637 \\
4,518 \\
5,821 \\
14,000\end{array}$ & $\begin{array}{l}1918 \ldots \\
1919 \ldots \\
1920 \ldots\end{array}$ & $\begin{array}{l}14,000 \\
14,000 \\
13,500\end{array}$ \\
\hline
\end{tabular}

1 The Dairy Division record of the number of creameries was discontinued with the year 1916. The later figures are estimated by the Bureau of Markets.

$$
75578^{\circ}-22-4
$$


TABLE 42.-Production of POWDERED MILK PRODUCTS in the United States for the year 1919, by months.

[Bureau of Markets and Crop Estimates.]

\begin{tabular}{|c|c|c|c|c|}
\hline Month. & $\begin{array}{l}\text { Whole- } \\
\text { milk } \\
\text { powder. }\end{array}$ & $\begin{array}{l}\text { Skim- } \\
\text { milk } \\
\text { powder. }\end{array}$ & $\begin{array}{c}\text { Cream } \\
\text { powder. }\end{array}$ & $\begin{array}{l}\text { Malted } \\
\text { milk. }\end{array}$ \\
\hline 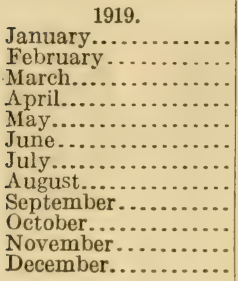 & $\begin{array}{r}\text { Pounds. } \\
277,426 \\
801,865 \\
739,185 \\
612,272 \\
1,093,084 \\
832,669 \\
1,036,194 \\
977,772 \\
868,465 \\
294,598 \\
522,974 \\
604,281\end{array}$ & $\begin{array}{l}\text { Pounds. } \\
1,606,854 \\
1,807,397 \\
2,899,490 \\
3,711,441 \\
4,506,141 \\
4,102,868 \\
3,343,393 \\
3,199,303 \\
2,372,636 \\
2,230,808 \\
1,472,701 \\
1,823,099\end{array}$ & $\begin{array}{r}\text { Pounds. } \\
49,685 \\
43,444 \\
69,338 \\
104,876 \\
53,424 \\
23,704 \\
68,458 \\
87,754 \\
38,832 \\
19,301 \\
230 \\
33,024\end{array}$ & $\begin{array}{l}\text { Pounds. } \\
1,649,002 \\
1,599,413 \\
1,835,121 \\
1,676,726 \\
1,423,864 \\
1,306,489 \\
1,178,746 \\
1,020,772 \\
1,143,352 \\
1,504,594 \\
1,596,889 \\
1,560,916\end{array}$ \\
\hline Total... & $8,660,785$ & $33,076,131$ & 592,070 & $17,495,887$ \\
\hline Month. & • & $\begin{array}{c}\text { Buttermilk } \\
\text { powder. }\end{array}$ & $\begin{array}{c}\text { Dried } \\
\text { casein } \\
\text { (lactarene).1 }\end{array}$ & $\begin{array}{l}\text { Crude milk } \\
\text { sugar } \\
\text { (lactose). }\end{array}$ \\
\hline 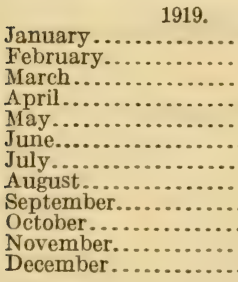 & $\begin{array}{c} \\
\cdots \\
\cdots \\
\cdots\end{array}$ & $\begin{array}{r}\text { Pounds. } \\
300,734 \\
237,685 \\
298,481 \\
399,891 \\
1,007,857 \\
786,146 \\
601,178 \\
438,554 \\
341,810 \\
349,694 \\
242,819 \\
273,978\end{array}$ & $\begin{array}{r}\text { Pounds. } \\
519,861 \\
718,024 \\
1,117,961 \\
1,972,017 \\
1,973,198 \\
1,905,882 \\
1,580,416 \\
1,104,200 \\
860,643 \\
822,604 \\
638,207 \\
711,823\end{array}$ & $\begin{array}{l}\text { Pounds. } \\
194,897 \\
204,625 \\
440,318 \\
592,848 \\
719,719 \\
799,639 \\
752,071 \\
656,987 \\
508,539 \\
502,899 \\
442,342 \\
406,458\end{array}$ \\
\hline Total. . & & $5,278,827$ & $13,924,836$ & $6,221,342$ \\
\hline
\end{tabular}

${ }_{1}$ Dried casein includes both skim-milk and buttermilk casein, the skım-milk casein amounting to $13,202,685$ pounds and the buttermilk casein to 722,151 pounds.

These figures represent the production of practically all the factories. 


\section{TABLE 43.-Quantities of MILK, BUTTER, and CHEESE produced on farms during 1919 and number of DAIRY COWS and YIELD of milk per,cow.}

\section{[Bureau of the Census.]}

\begin{tabular}{|c|c|c|c|c|c|}
\hline State. & $\begin{array}{l}\text { Milk pro- } \\
\text { duced on } \\
\text { farms. }\end{array}$ & $\begin{array}{c}\text { Farm } \\
\text { butter. }\end{array}$ & $\begin{array}{l}\text { Farm } \\
\text { cheese. }\end{array}$ & $\begin{array}{l}\text { Dairy } \\
\text { cows. }\end{array}$ & $\begin{array}{l}\text { Yield } \\
\text { per } \\
\text { cow. }\end{array}$ \\
\hline & Gallons. & Pounds. & Pounds. & & Lbs. \\
\hline & $\begin{array}{l}93,903,677 \\
14,370,833\end{array}$ & $28,490,181$ & 5, 210 & & 1,944 \\
\hline & $87,623,651$ & $25,571,098$ & $\begin{array}{r}90,119 \\
8,603\end{array}$ & 415 & $\begin{array}{l}3,724 \\
1,634\end{array}$ \\
\hline & $276,424,216$ & $5,757,759$ & 2,345, & 502 & 4,610 \\
\hline & $79,492,631$ & $5,775,602$ & 157,186 & 192,234 & 2,993 \\
\hline & 54,8 & $1,926,127$ & 90,500 & 112,622 & 4,128 \\
\hline & $11,356,313$ & 894,883 & 3,170 & 33,026 & 2,92 \\
\hline & 512,074 & 6,020 & & & 6,21 \\
\hline & $2,155,533$ & $1,162,3$ & & 71,641 & \\
\hline & 5,773 & 30,2 & 1,3 & 388,448 & 12 \\
\hline & 52 & 4,5 & 31,6 & 115 & 560 \\
\hline & 370 , & $25, \mathrm{C}$ & 117 , & & 04 \\
\hline Ind & 2 & 1 & 109 & & 3,04 \\
\hline & & & & & 2,70 \\
\hline & 2 & 17 & 19, & & 31 \\
\hline & & & 13 , & 441 , & 2,60 \\
\hline & 32 , & & & 176, & 1,3 \\
\hline & 77 , & 10 & $55^{\prime}$ & & 7 \\
\hline & 58,7 & 6,1 & 126,1 & & 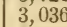 \\
\hline & & & 60 & 147 & 1,35 \\
\hline & 382 & 25, & & & 3,96 \\
\hline & 47 & 20 & 71 , & 1, 229, & 3,0 \\
\hline & & 2 & 2 , & 427 & 1,5 \\
\hline & 228 & 29 & 90 & 661, & 2,33 \\
\hline & 51,2 & & 43 , & 127,5 & 2,86 \\
\hline & 16 & 13 , ? & 23 , & 438,4 & 2,5 \\
\hline & $6,312,10$ & $5,($ & 22, & 13 & 3,8 \\
\hline & $42,556,28$ & 3,2 & 32 , & 95,9 & 3,68 \\
\hline & & & 92, & 130 & 3,02 \\
\hline & & & 87, & & 2,21 \\
\hline & 756,0 & 24,7 & 521, & 1,481, & 4,31 \\
\hline ina & 9 & 25,5 & 16,1 & 290 , & 2,66 \\
\hline $\mathrm{N}$ & & 14,4 & 50 & & 2,65 \\
\hline & & & 434 , & & 3,60 \\
\hline & & 22,2 & & & 2,01 \\
\hline & 92 & 4,1 & & & 4,13 \\
\hline & & 38,4 & 532,2 & $885, \mathrm{~s}$ & 3,9 \\
\hline & 12 , & 2 & 10,4 & 21,4 & 4,73 \\
\hline & & & & & 2,3 \\
\hline & & & & & 2,33 \\
\hline & & & 114 & & 2,49 \\
\hline & 202,9 & 49,40 & 107, & 833, & 1,8 \\
\hline & & & 73 & 66 , & 3,46 \\
\hline & $122,095,7$ & & & & 3,56 \\
\hline & & 25,47 & 39 , & & 2,51 \\
\hline & 140,52 & & & & 4,91 \\
\hline & - & 17,7 & $8 S, 5$ & 181 , & 2,98 \\
\hline & 858,2 & 8,60 & 308,117 & $1,795,122$ & 4,016 \\
\hline Wyom & $14,613,581$ & $1,422,822$ & 6,837 & 34,997 & 2,692 \\
\hline Tot & & $707,666,492$ & & & 3,412 \\
\hline
\end{tabular}




\section{TABLE 44.-CREAMERY BUTTER in STORAGE on the first of each month.}

[Bureau of Markets and Crop Estimates. Thousand pounds-000 omitted.]

\begin{tabular}{|c|c|c|c|c|c|c|c|}
\hline Month. & 1915 & 1916 & 1917 & 1918 & 1919 & 1920 & 1921 \\
\hline & Pounds. & Pounds. & Pounds. & Pounds. & Pounds. & Pounds. & Pounds. \\
\hline Jan. & & 48,977 & 46,134 & 50,726 & 43,910 & 53,737 & 58,682 \\
\hline & & 31,139 & 30,474 & 26,618 & 36,777 & 38,359 & 41,486 \\
\hline $\begin{array}{l}\text { Mar } \\
\text { Apr }\end{array}$ & & 15,033 & 16,952 & 18, & 24,191 & 22, & 27,103 \\
\hline Apr & & 3,346 & 6,8 & 14,629 & 11,909 & 12,555 & 14,732 \\
\hline May. & & 1,082 & 3,60 & 9,536 & 9,659 & 7,554 & 7,712 \\
\hline June. & & 7,017 & 9,953 & 12,698 & 29,435 & 12,872 & 21,682 \\
\hline & & 53,863 & 49,982 & 49,140 & 90,158 & 52,526 & 62,168 \\
\hline Aug. & 68,578 & 102,537 & 88,99 & 88,305 & 123,546 & 101,455 & 82,838 \\
\hline Sept. & 101,662 & 105,836 & 108,1 & $99,33 \pm$ & 131,388 & & 92,396 \\
\hline Oct.. & 99,450 & 100,522 & 109,15 & 87,883 & 121,816 & 113,385 & 90,123 \\
\hline Nov. & 92,719 & 85,260 & 100,11 & 80,874 & 100,474 & 101,778 & 77,893 \\
\hline Dec... & 71,849 & 67,292 & 79,928 & 65,111 & 73,654 & 79,750 & 65,138 \\
\hline
\end{tabular}

NoTE.-Cold storage in connection with butter in this table means the holding of butter at a temperature of $45^{\circ} \mathrm{F}$. or less for a period of at least 30 days.

\section{TABLE 45.-RECEIPTS of BUTTER on New York, Chi- cago, and San Francisco markets.}

[Trade journals and Bureau of Markets and Crop Estimates.2]

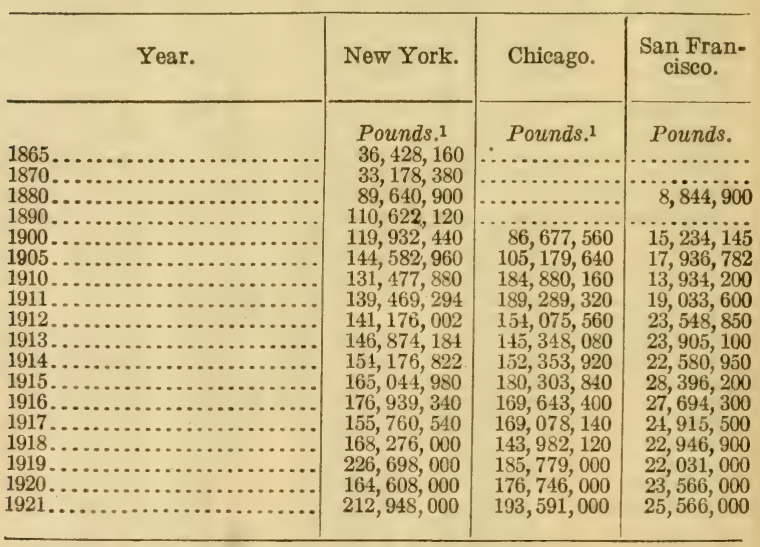

1 Estimated at 60 pounds per tub. $\quad 2$ Bureau reports began in 1919. 


\section{TABLE 46.-IMPORTS and EXPORTS of BUTTER.}

[Commerce and Navigation Reports; (YB.)]

\begin{tabular}{|c|c|c|c|c|}
\hline Year ended June $30-$ & \multicolumn{2}{|c|}{ General imports. 1} & \multicolumn{2}{|c|}{ Domestic exports.1 } \\
\hline $\begin{array}{l}1851 \\
1860 \\
1870 \\
1880 \\
1890 \\
1900 \\
1910 \\
1911 \\
1912 \\
1912 \\
1913 \\
1914 \\
1915 \\
1916 \\
1916 \\
1917 \\
1918 \\
1919 \\
1919 \\
1920 \\
1920 \\
1921\end{array}$ & $\begin{array}{r}\text { Pounds. } \\
479,180 \\
3,278,967 \\
4,089,038 \\
487,120 \\
75,521 \\
49,791 \\
1,360,245 \\
1,007,826 \\
1,025,668 \\
1,162,253 \\
7,842,022 \\
3,829,227 \\
712,998 \\
523,573 \\
1,805,925 \\
4,131,469 \\
20,770,959 \\
37,454,172 \\
18,558,388\end{array}$ & $\begin{array}{r}\text { Value. } \\
\$ 37,536 \\
506,185 \\
832,829 \\
95,122 \\
13,679 \\
9,769 \\
298,023 \\
247,961 \\
237,154 \\
304,090 \\
1,753,461 \\
977,262 \\
212,370 \\
192,767 \\
619,303 \\
1,869,132 \\
10,916,770 \\
18,646,456 \\
7,392,247\end{array}$ & $\begin{array}{r}\text { Pounds. } \\
3,994,542 \\
7,640,914 \\
2,019,288 \\
39,236,658 \\
29,748,042 \\
13,266,371 \\
3,140,545 \\
4,877,797 \\
6,092,235 \\
3,585,600 \\
3,693,597 \\
9,850,704 \\
13,487,481 \\
26,835,092 \\
17,735,966 \\
33,739,960 \\
27,155,834 \\
17,487,735 \\
8,014,737\end{array}$ & $\begin{array}{r}\text { Value. } \\
\$ 516,812 \\
1,144,321 \\
592,229 \\
6,690,687 \\
4,187,489 \\
3,143,509 \\
785,771 \\
1,059,432 \\
1,468,432 \\
872,804 \\
877,453 \\
2,392,480 \\
3,590,105 \\
8,749,170 \\
6,852,727 \\
15,843,522 \\
15,491,682 \\
10,142,403 \\
3,269,609\end{array}$ \\
\hline
\end{tabular}

1 See explanatory notes at beginning of bulletin.

2 Year ended Dec. 31. The Commerce and Navigation Reports are now made by years ending Dec. 31

\section{TABLE 47.-Average weekly QUOTATIONS for highest grade of BUTTER on the San Francisco market.}

[Pacific Dairy Review and Bureau of Markets and Crop Estimates.1]

\begin{tabular}{|c|c|c|c|c|c|}
\hline Year. & $\begin{array}{l}\text { Averge } \\
\text { price per } \\
\text { pound. }\end{array}$ & Year. & $\begin{array}{c}\text { Average } \\
\text { price per } \\
\text { pound. }\end{array}$ & Year. & $\begin{array}{l}\text { Average } \\
\text { price per } \\
\text { pound. }\end{array}$ \\
\hline $\begin{array}{l}1899 \ldots \\
1900 \ldots \\
1901 \ldots \\
1902 \ldots \\
1903 \ldots \\
1904 \ldots \\
1905 \ldots \\
1906 \ldots\end{array}$ & $\begin{array}{r}\text { Cents. } \\
22.9 \\
23.0 \\
21.2 \\
25.5 \\
27.9 \\
23.5 \\
24.7 \\
25.8\end{array}$ & $\begin{array}{l}1907 \ldots \\
1908 \ldots \\
1909 \\
1910 \ldots \\
1911 \ldots \\
1912 \ldots \\
1913 \ldots \\
1914 \ldots\end{array}$ & $\begin{array}{r}\text { Cents. } \\
30.5 \\
27.4 \\
30.6 \\
31.4 \\
27.1 \\
31.2 \\
31.7 \\
27.0\end{array}$ & $\begin{array}{l}1915 \ldots \ldots \\
1916 \ldots \ldots \\
1917 \ldots \ldots \\
1918 \ldots \ldots \\
1919 \ldots \ldots \\
1920 \ldots \ldots \\
1921 \ldots \ldots\end{array}$ & $\begin{array}{r}\text { Cents. } \\
26.3 \\
28.9 \\
39.9 \\
52.2 \\
57 \\
60.2 \\
36.3\end{array}$ \\
\hline
\end{tabular}

1 Prices by Bureau of Markets and Crop Estimates began in 1919. 
TABLE 48.-Average monthly PRICE of CREAMERY BUTTER, extras or equivalent grade, on New York market.

[New York Produce Review and Bureau of Marketsand Crop Estimates.]

\begin{tabular}{|c|c|c|c|c|c|c|c|c|}
\hline \multicolumn{2}{|l|}{ Month. } & 1900 & 1901 & 1902 & 1903 & 1904 & 1905 & 1906 \\
\hline \multicolumn{2}{|c|}{ 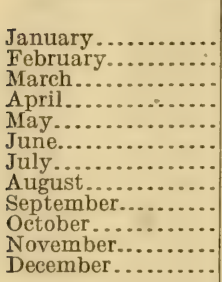 } & \begin{tabular}{|r|} 
Cents. \\
26.8 \\
24.9 \\
24.9 \\
16.3 \\
20.1 \\
19.4 \\
19.5 \\
20.8 \\
21.5 \\
21.8 \\
24.9 \\
25.3
\end{tabular} & $\begin{array}{r}\text { Cents. } \\
22.8 \\
22.5 \\
22.1 \\
21.0 \\
19.0 \\
19.3 \\
19.7 \\
20.3 \\
20.9 \\
20.0 \\
24.1 \\
25.0\end{array}$ & $\begin{array}{r}\text { Cents. } \\
24.1 \\
28.3 \\
28.2 \\
28.8 \\
22.7 \\
22.0 \\
21.3 \\
19.9 \\
21.6 \\
24.0 \\
26.6 \\
29.1\end{array}$ & $\begin{array}{r}\text { Cents. } \\
27.3 \\
26.6 \\
28.4 \\
26.6 \\
22.0 \\
21.7 \\
20.1 \\
19.3 \\
20.6 \\
21.3 \\
23.1 \\
24.2\end{array}$ & $\begin{array}{l}\text { Cents. } \\
22.7 \\
25.1 \\
24.5 \\
22.8 \\
20.1 \\
18.0 \\
17.6 \\
17.9 \\
19.4 \\
20.9 \\
24.8 \\
26.8\end{array}$ & $\begin{array}{r}\text { Cents. } \\
29.1 \\
32.1 \\
28.0 \\
30.0 \\
23.7 \\
20.4 \\
20.5 \\
21.1 \\
20.6 \\
21.8 \\
23.6 \\
24.3\end{array}$ & $\begin{array}{r}\text { Cents. } \\
26.5 \\
27.1 \\
27.0 \\
21.8 \\
20.1 \\
20.2 \\
20.6 \\
22.5 \\
24.6 \\
26.1 \\
27.6 \\
31.6\end{array}$ \\
\hline \multicolumn{2}{|c|}{ Average......... } & 22.2 & 21.4 & 24.7 & 23.4 & 21.7 & 24.6 & 24.6 \\
\hline \multicolumn{2}{|l|}{ Month. } & 1907 & 1908 & 1909 & 1910 & 1911 & 1912 & 1913 \\
\hline \multicolumn{2}{|c|}{ 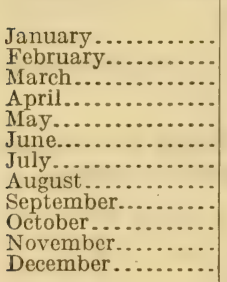 } & $\begin{array}{r}\text { Cents. } \\
30.8 \\
32.5 \\
30.6 \\
30.6 \\
25.0 \\
23.6 \\
24.8 \\
125.5 \\
28.3 \\
29.2 \\
27.7 \\
29.8\end{array}$ & $\begin{array}{l}\text { Cents. } \\
31.5 \\
32.8 \\
29.3 \\
29.0 \\
24.2 \\
23.8 \\
22.9 \\
23.3 \\
24.5 \\
27.4 \\
30.3 \\
32.0\end{array}$ & $\begin{array}{l}\text { Cents. } \\
32.2 \\
31.0 \\
30.0 \\
27.9 \\
27.0 \\
26.3 \\
26.7 \\
27.7 \\
31.0 \\
31.6 \\
31.9 \\
36.0\end{array}$ & $\begin{array}{r}\text { Cents. } \\
34.4 \\
30.6 \\
33.5 \\
32.0 \\
29.0 \\
28.5 \\
29.1 \\
30.3 \\
31.2 \\
31.4 \\
32.5 \\
30.9\end{array}$ & $\begin{array}{r}\text { Cents. } \\
28.0 \\
28.0 \\
25.5 \\
22.1 \\
22.8 \\
23.8 \\
25.9 \\
27.3 \\
27.6 \\
31.4 \\
34.8 \\
37.8\end{array}$ & $\begin{array}{r}\text { Cents. } \\
39.1 \\
31.7 \\
31.1 \\
32.8 \\
230.4 \\
27.3 \\
27.1 \\
26.6 \\
29.7 \\
31.2 \\
34.4 \\
37.2\end{array}$ & $\begin{array}{r}\text { Cents. } \\
35.1 \\
36.3 \\
36.7 \\
34.5 \\
28.6 \\
27.8 \\
27.0 \\
27.9 \\
31.5 \\
31.4 \\
33.8 \\
36.1\end{array}$ \\
\hline \multicolumn{2}{|c|}{ Average........ } & 28.1 & 27.6 & 29.9 & 31.1 & 27.9 & 31.6 & 32.2 \\
\hline Month. & 1914 & 1915 & 1916 & 1917 & ${ }^{3} 1918$ & 1919 & 1920 & 1921 \\
\hline $\begin{array}{l}\text { October ..... } \\
\text { November... } \\
\text { December ... }\end{array}$ & $\begin{array}{r}\text { Cents. } \\
32.6 \\
29.3 \\
27.7 \\
25.4 \\
26.1 \\
27.2 \\
27.9 \\
30.4 \\
31.4 \\
31.6 \\
34.7 \\
33.9\end{array}$ & $\begin{array}{r}\text { Cents. } \\
33.5 \\
32.3 \\
29.7 \\
30.7 \\
28.8 \\
28.2 \\
27.0 \\
25.9 \\
26.6 \\
28.6 \\
31.1 \\
35.1\end{array}$ & $\begin{array}{r}\text { Cents. } \\
32.6 \\
33.8 \\
36.9 \\
36.0 \\
31.0 \\
29.8 \\
28.9 \\
31.2 \\
33.9 \\
35.4 \\
39.4 \\
39.8\end{array}$ & $\begin{array}{r}\text { Cents. } \\
40.1 \\
43.5 \\
41.6 \\
44.2 \\
40.2 \\
39.1 \\
39.0 \\
41.3 \\
44.3 \\
44.6 \\
45.5 \\
49.4\end{array}$ & $\begin{array}{r}\text { Cents. } \\
52 \\
50 \\
44 \\
42 \\
42 \\
44 \\
45 \\
46 \\
56 \\
59 \\
63 \\
69\end{array}$ & $\begin{array}{r}\text { Cents. } \\
62 \\
52 \\
62 \\
64 \\
58 \\
52 \\
53 \\
55 \\
59 \\
68 \\
71 \\
72\end{array}$ & \begin{tabular}{|r} 
Cents. \\
65 \\
66 \\
67 \\
71 \\
61 \\
57 \\
57 \\
55 \\
59 \\
60 \\
63 \\
55
\end{tabular} & $\begin{array}{r}\text { Cents. } \\
52.5 \\
47.2 \\
48.1 \\
45.5 \\
31.8 \\
32.7 \\
40.4 \\
42.7 \\
43.1 \\
47.0 \\
44.9 \\
43.8\end{array}$ \\
\hline Average.. & 29.8 & 29.8 & 34.9 & 42.7 & 51 & 61 & 61 & 43.3 \\
\hline
\end{tabular}

1 Aug. 5, 1907, "extras" changed to "specials."

2 "Extras" and "firsts" took place of "specials" May 13, 1912.

3 Prices by Bureau of Markets and Crop Estimates began in 1918. 


\section{1}

\section{TABLE 49.-AVERAGE PRICE of BUTTER on New York market-grade of extras.}

[NewYork Produce Review and Bureau of Mtarkets and Crop Estimates.]

\begin{tabular}{|c|c|c|c|c|c|c|c|c|}
\hline \multirow[b]{2}{*}{ Year. } & \multicolumn{2}{|c|}{ Average price. } & \multirow[b]{2}{*}{ Year. } & \multicolumn{2}{|c|}{ Average price. } & \multirow[b]{2}{*}{ Year. } & \multicolumn{2}{|c|}{ Average price. } \\
\hline & $\begin{array}{c}\text { Mar- } \\
\text { ket } \\
\text { quo- } \\
\text { ta- } \\
\text { tion. }\end{array}$ & $\begin{array}{l}\text { Price in } \\
\text { gold } \\
\text { during } \\
\text { period } \\
\text { of cur- } \\
\text { rency } \\
\text { infla- } \\
\text { tion. }\end{array}$ & & $\begin{array}{l}\text { Mar- } \\
\text { ket } \\
\text { quo- } \\
\text { ta- } \\
\text { tion. }\end{array}$ & $\begin{array}{c}\text { Price in } \\
\text { gold } \\
\text { during } \\
\text { period } \\
\text { of cur- } \\
\text { rency } \\
\text { infla- } \\
\text { tion. }\end{array}$ & & $\begin{array}{l}\text { Mar- } \\
\text { ket } \\
\text { quo- } \\
\text { ta- } \\
\text { tion. }\end{array}$ & $\begin{array}{l}\text { Price in } \\
\text { gold } \\
\text { during } \\
\text { period } \\
\text { of cur- } \\
\text { rency } \\
\text { infla- } \\
\text { tion. }\end{array}$ \\
\hline & Cents. & Cents. & & Cents. & & & Cents & Cents. \\
\hline 1866. & 51.1 & 36.3 & 1885. & 26.8 & & $1904 .$. & 21.7 & ........... \\
\hline & 41.6 & 30.1 & & 27.4 & & 1905. & 24.6 & $\cdots$ \\
\hline & $\begin{array}{l}49 . \\
47 .\end{array}$ & $\begin{array}{l}35 . \\
35 .\end{array}$ & $\begin{array}{l}185 \\
188\end{array}$ & $\begin{array}{l}26.9 \\
27.3\end{array}$ & & 7. & $\begin{array}{l}24.0 \\
28.1\end{array}$ & \\
\hline & 40. & 35 . & 188 & 24.3 & & 190 & 27.6 & \\
\hline & 35. & & & 23. & & & 29.9 & \\
\hline & 34. & 30 & 189 & 26.2 & & 191 & 31.1 & \\
\hline & 39 & 34 & & 26. & & 191 & 27.4 & \\
\hline & 41 & & & 27 . & & & 31.6 & \\
\hline & 34. & 30. & 1894 . & 22 . & & 191 & 32.2 & \\
\hline & 34. & 31 & 185 & 21. & & 191 & 29.8 & \\
\hline & & & & 18 & & & 29.8 & \\
\hline & 28. & 28. & & 19 & & 191 & 34.9 & \\
\hline & 25.5 & & & 19. & & $1917 .$. & 42.7 & \\
\hline 1 & 30.5 & & & 21.2 & & 1918. & 151.0 & \\
\hline & 32. & & 190 & 22. & & 191 & 61.0 & \\
\hline & 35. & & 1901 & 21. & & 1920 & 61.0 & \\
\hline & 31. & & 1902. & 24. & & 1921. & 43.3 & \\
\hline & 30.1 & & 1903. & 23.4 & & & & \\
\hline
\end{tabular}

1 Prices by Bureau of Markets and Crop Estimates began in 1918.

\section{TABLE 50.-CHEESE production in the United States by kinds.}

[Bureau of Markets and Crop Estimates.]

\begin{tabular}{|c|c|c|c|c|c|}
\hline $\begin{array}{c}\text { Kinds of } \\
\text { cheese made. }\end{array}$ & 1916 & 1917 & 1918 & 1919 & 1920 \\
\hline $\begin{array}{l}\text { Joeddar: } \\
\text { Vhole mill. } \\
\text { art skim.. } \\
\text { 5ull skim .. } \\
\text { Swiss........ } \\
\text { Limburger.... } \\
\text { All Italian.... } \\
\text { B r i c k and } \\
\text { Münster.... } \\
\text { C r e a m and } \\
\text { Neufehâtel . } \\
\text { All others..... }\end{array}$ & \begin{tabular}{|r|} 
Pounds. \\
$227,032,325$ \\
$7,856,189$ \\
$4,726,450$ \\
$15,317,466$ \\
$9,623,315$ \\
Not given. \\
$41,552,388$
\end{tabular} & \begin{tabular}{|r|} 
Pounds. \\
$274,945,880$ \\
$8,058,216$ \\
$5,149,557$ \\
$15,435,097$ \\
$9,798,247$ \\
Not given. \\
$45,922,624$
\end{tabular} & \begin{tabular}{|r|}
\multicolumn{1}{|c|}{ Pounds. } \\
$247,276,503$ \\
$9,102,159$ \\
$6,610,993$ \\
$18,586,505$ \\
$8,808,140$ \\
$2,042,234$ \\
\\
$43,332,215$
\end{tabular} & \begin{tabular}{|r|}
\multicolumn{1}{|c|}{ Pounds. } \\
$2 \mathrm{~s} 1,836,015$ \\
$6,189,192$ \\
$6,985,356$ \\
$21,601,782$ \\
$7,625,541$ \\
$3,987,361$ \\
$37,521,004$
\end{tabular} & $\begin{array}{r}\text { Pounds. } \\
242,195,707 \\
4,346,400 \\
6,365,267 \\
20,430,000 \\
7,170,388 \\
4,677,525 \\
40,687,832\end{array}$ \\
\hline
\end{tabular}

The Italian, Cream, and Neufchâtel cheeses are included in "All others" for the years 1916 and 1917. 


\section{TABLE 51.-Total PRODUCTION of farm and factory CHEESE in the United States.}

[Census Bureau and Bureau of Markets and Crop Estimates$\mathrm{c}=$ census. $\mathrm{m}=$ markets.]

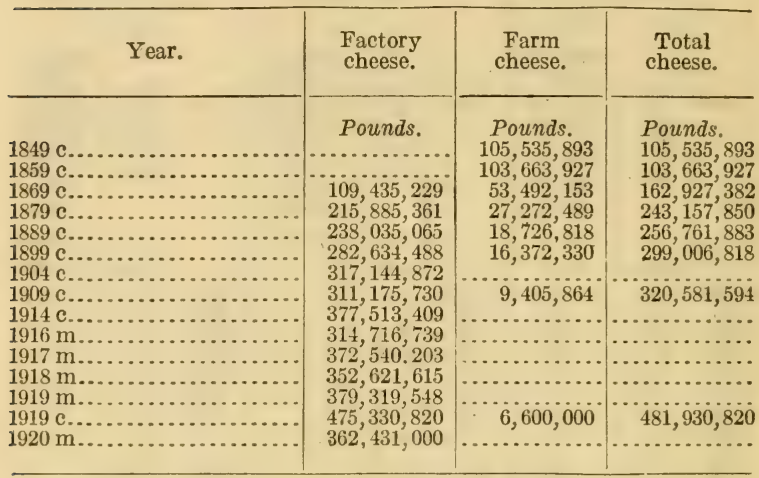

There are no records of farm-made cheese except for census years.

For production of cheese by States see Tables 38, 39, and 43.

Cottage cheese is included in census figures but not in markets figures.

\section{TABLE 52.-DOMESTIC SWISS CHEESE production for 1919 and 1920, by States.}

[Bureau of Markets and Crop Estimates.]

\begin{tabular}{|c|c|c|c|}
\hline \multirow{2}{*}{ State. } & \multirow{2}{*}{$\begin{array}{l}\text { Num- } \\
\text { ber of } \\
\text { facto- } \\
\text { ries. }\end{array}$} & \multicolumn{2}{|c|}{ Production. } \\
\hline & & 1919 & 1920 \\
\hline 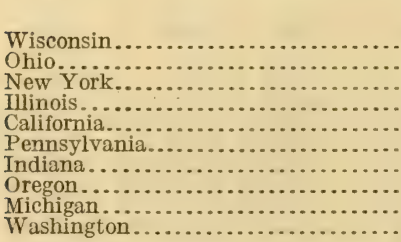 & $\begin{array}{r}232 \\
56 \\
11 \\
23 \\
3 \\
3 \\
1 \\
1 \\
1 \\
1\end{array}$ & $\begin{array}{r}\text { Pounds. } \\
14,328,204 \\
3,030,357 \\
1,528,704 \\
947,526 \\
938,694 \\
813,041 \\
12,336 \\
1,610 \\
1,300 \\
10\end{array}$ & 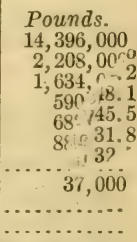 \\
\hline Total...... & 353 & $21,601,782$ & $20,430,000$ \\
\hline
\end{tabular}

1 These are all the factories producing domestic Swiss cheese 
TABLE 53.-Amount and value of CHEESE IMIPORTED into the United States from France, Greece, Italy, and Switzerland.

[Commerce and Navigation Reports.]

\begin{tabular}{|c|c|c|c|c|}
\hline \multirow{2}{*}{$\begin{array}{l}\text { Year ended } \\
\text { June } 30-\end{array}$} & \multicolumn{2}{|c|}{ France. } & \multicolumn{2}{|c|}{ Greece. } \\
\hline & Pounds. & Value. & Pounds. & Value. \\
\hline $\begin{array}{l}910 \ldots \\
911 \ldots \\
912 \ldots \\
913 \ldots \ldots \\
914 \ldots \\
915 \ldots \ldots \\
916 \ldots \\
917 \ldots \ldots \\
918 \ldots \ldots \\
919 \ldots \ldots \\
920 \ldots \ldots \\
921 \ldots \ldots \\
\end{array}$ & $\begin{array}{r}3,673,117 \\
3,756,155 \\
3,882,891 \\
3,982,513 \\
5,418,904 \\
3,554,297 \\
2,321,543 \\
1,937,341 \\
1,026,117 \\
404,722 \\
810,948 \\
2,417,036\end{array}$ & $\begin{array}{r}\$ 704,685 \\
728,126 \\
942,683 \\
785,965 \\
1,032,817 \\
737,212 \\
784,323 \\
754,012 \\
528,926 \\
349,331 \\
396,977 \\
926,343\end{array}$ & $\begin{array}{r}1,777,819 \\
2,598,474 \\
2,703,745 \\
2,089,079 \\
3,212,908 \\
3,003,349 \\
1,132,200 \\
83,632 \\
\text { None. } \\
\text { None. } \\
7,366 \\
84,716\end{array}$ & $\begin{array}{r}\$ 230,031 \\
343,678 \\
409,990 \\
321,182 \\
447,124 \\
402,952 \\
169,536 \\
18,089 \\
\ldots . . . . . \\
2,801 \\
34,028\end{array}$ \\
\hline Total...... & $33,185,584$ & & $16,693,288$ & $2,379,411$ \\
\hline \multirow{2}{*}{$\begin{array}{l}\text { Year ended } \\
\text { June } 30-\end{array}$} & \multicolumn{2}{|c|}{ Italy. } & \multicolumn{2}{|c|}{ Switzerland. } \\
\hline & Pounds. & Value. & Pounds. & Value. \\
\hline $1920 \ldots \ldots$ & $\begin{array}{r}17,122,124 \\
18,647,412 \\
20,625,202 \\
21,326,445 \\
26,453,626 \\
25,662,362 \\
16,084,058 \\
8,482,280 \\
16,044 \\
57 \\
1,095,740 \\
1,185,912\end{array}$ & $\begin{array}{r}\$ 3,175,640 \\
3,381,546 \\
3,948,550 \\
4,217,674 \\
5,024,270 \\
5,108,850 \\
3,855,856 \\
2,545,286 \\
7,883 \\
23 \\
274,970 \\
396,484\end{array}$ & 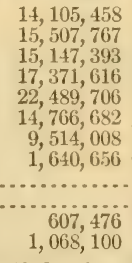 & $\begin{array}{r}\$ 2,372,134 \\
2,758,851 \\
2,857,631 \\
3,183,350 \\
3,617,657 \\
2,677,249 \\
2,031,590 \\
341,063 \\
\ldots . . . . . . \\
\ldots . . .6 . \\
301,239 \\
399,329\end{array}$ \\
\hline Total...... & $156,701,262$ & $35,792,882$ & $112,218,862$ & $20,443,096$ \\
\hline
\end{tabular}

TABLE 54.-IMPORTS and EXPORTS of CHEESE.

[Commerce and Navigation Reports; (YB.).]

\begin{tabular}{|c|c|c|c|c|c|}
\hline $\begin{array}{c}\text { Year } \\
\text { ended } \\
\text { June } 30-\end{array}$ & Imports. & Exports. & $\begin{array}{c}\text { Year } \\
\text { ended } \\
\text { June } 30-\end{array}$ & Imports. & Exports. \\
\hline $\begin{array}{l}1851 \ldots \ldots \\
1860 \ldots \ldots \\
1870 \ldots \ldots \\
1880 \ldots \ldots \\
1881{ }^{1} \ldots \ldots \\
1890 \ldots \ldots \\
1900 \ldots \ldots \\
1910 \ldots \ldots \\
1911 \ldots \ldots \\
1912 \ldots \ldots\end{array}$ & $\begin{array}{r}\text { Pounds. } \\
603,398 \\
1,401,161 \\
2,289,257 \\
2,737,186 \\
2,655,370 \\
9,263,573 \\
13,455,990 \\
40,817,524 \\
45,568,797 \\
46,542,007\end{array}$ & $\begin{array}{r}\text { Pounds. } \\
10,361,189 \\
15,515,799 \\
57,296,327 \\
127,553,907 \\
147,995,614 \\
95,376,053 \\
48,419,353 \\
2,846,709 \\
10,366,605 \\
6,337,559\end{array}$ & $\begin{array}{l}1913 \ldots \ldots \\
19144^{2} \ldots \\
1915 \ldots \\
1916 \ldots \\
1917 \ldots \\
1918 \ldots \\
1919 \ldots \\
1920 \ldots \\
1920^{3} \ldots\end{array}$ & $\begin{array}{r}\text { Pounds. } \\
49,387,994 \\
63,784,313 \\
50,138,520 \\
30,087,999 \\
14,481,514 \\
9,839,305 \\
2,442,306 \\
17,913,682 \\
15,993,725\end{array}$ & $\begin{array}{r}\text { Pounds. } \\
2,599,058 \\
2,427,577 \\
55,362,917 \\
44,394,301 \\
66,050,013 \\
44,330,978 \\
18,794,853 \\
19,378,158 \\
16,291,319\end{array}$ \\
\hline
\end{tabular}

${ }^{1}$ Largest exports. ${ }^{2}$ Largest imports. ${ }^{3}$ Year ended Dec. 31.

$75578^{\circ}-22-5$ 
TABLE 55.-Highest average daily quotations on AMERICAN CHEDDAR CHEESE in New York State and New York City.

[Producers' Price Current, and New York Produce Review. Bureau of Markets and Crop Estimates.]

\begin{tabular}{|c|c|c|c|c|c|}
\hline Year. & $\begin{array}{c}\text { Price } \\
\text { per } \\
\text { pound. }\end{array}$ & Year. & $\begin{array}{c}\text { Price } \\
\text { per } \\
\text { pound. }\end{array}$ & Year. & $\begin{array}{c}\text { Price } \\
\text { per } \\
\text { pound. }\end{array}$ \\
\hline $\begin{array}{l}1851 \ldots \ldots \\
1860 \ldots \ldots \\
1870 \ldots \ldots \\
1880 \ldots \ldots \\
1890 \ldots \ldots \\
1900 \ldots \ldots\end{array}$ & $\begin{array}{r}\text { Cents. } \\
5.7 \\
11.0 \\
119.2 \\
13.7 \\
9.5 \\
11.2\end{array}$ & $\begin{array}{l}1910 \ldots \ldots \\
1911 \ldots \ldots \\
1912 \ldots \ldots \\
1913 \ldots \ldots \\
1914 \ldots \ldots \\
1915 \ldots \ldots\end{array}$ & $\begin{array}{r}\text { Cents. } \\
16.4 \\
14.1 \\
16.4 \\
15.4 \\
15.6 \\
15.5\end{array}$ & $\begin{array}{l}1916 \ldots \ldots \\
1917 \ldots \ldots \\
1918 . \ldots \ldots \\
1919 \ldots \ldots \\
1920 \ldots \ldots\end{array}$ & $\begin{array}{r}\text { Cents. } \\
19.0 \\
24.4 \\
27.2 \\
32.2 \\
29.0\end{array}$ \\
\hline
\end{tabular}

1 Price in gold 16.7 cents.

The quotations for 1851 and 1860 are from market centers outside of New York City. From 1870 on they are from New York City. For 1919 and 1920 from Bureau of Markets and Crop Estimates.

\section{TABLE 56.-Highest average weekly quotations on DO- MESTIC SWISS CHEESE (round) on the Chicago market.}

[Butter, Cheese and Egg Journal.]

\begin{tabular}{|c|c|c|c|c|c|}
\hline Year. & $\begin{array}{c}\text { Price } \\
\text { per } \\
\text { pound. }\end{array}$ & Year. & $\begin{array}{c}\text { Price } \\
\text { per } \\
\text { pound. }\end{array}$ & Year. & $\begin{array}{c}\text { Price } \\
\text { per } \\
\text { pound. }\end{array}$ \\
\hline $1903 \ldots$ & $\begin{array}{r}\text { Cents. } \\
12.79 \\
13.19 \\
13.06 \\
12.70 \\
11.76 \\
11.72 \\
12.64\end{array}$ & $\begin{array}{l}1907 \ldots \ldots \\
1908 . \ldots \ldots \\
1909 \ldots \ldots \\
1910 \ldots \ldots \\
1911 \ldots \ldots \\
1912 \ldots \ldots \\
1913 . \ldots \ldots\end{array}$ & $\begin{array}{r}\text { Cents. } \\
15.35 \\
12.09 \\
15.48 \\
18.87 \\
15.04 \\
18.66 \\
18.51\end{array}$ & $\begin{array}{l}1914 \ldots \ldots \\
1915 \ldots \ldots \\
1916 \ldots \ldots \\
1917 \ldots \ldots \\
1918 \ldots \\
1919 . \ldots \\
1920 \ldots \ldots\end{array}$ & $\begin{array}{r}\text { Cents. } \\
16.79 \\
18.57 \\
29.07 \\
36.23 \\
43.60 \\
53.32 \\
48.55\end{array}$ \\
\hline
\end{tabular}

TABLE 57.-PRODUCTION and EXPORTATION of OLEOMARGARIN for the United States.

[Bureau of Internal Revenue.]

\begin{tabular}{|c|c|c|c|c|c|}
\hline $\begin{array}{c}\text { Year } \\
\text { ended } \\
\text { Jan. } 30 .\end{array}$ & Production. & $\begin{array}{c}\text { Exporta- } \\
\text { tion. }\end{array}$ & $\begin{array}{c}\text { Year } \\
\text { ended } \\
\text { Jan. } 30 .\end{array}$ & Production. & $\begin{array}{c}\text { Exporta- } \\
\text { tion. }\end{array}$ \\
\hline $\begin{array}{l}1911 \ldots \\
1912 \ldots \\
1913 \ldots\end{array}$ & $\begin{array}{r}\text { Pounds. } \\
21,513,537 \\
32,324,032 \\
107,045,028 \\
51,987,336 \\
141,862,280 \\
121,162,795 \\
125,601,053 \\
145,227,862\end{array}$ & $\begin{array}{l}\text { Pounds. } \\
834,574 \\
2,535,926 \\
4,256,067 \\
2,543,433 \\
2,864,770 \\
3,146,094 \\
3,150,2 \$ 2 \\
2,477,659\end{array}$ & $\begin{array}{l}1914 \ldots \ldots \\
1915 \ldots \ldots \\
1916 \ldots \ldots \\
1917 \ldots \ldots \\
1918 \ldots \ldots \\
19^{\circ} 9 \ldots \ldots \\
1920 \ldots \ldots \\
1921 \ldots \ldots\end{array}$ & $\begin{array}{c}\text { Pounds. } \\
144,021,276 \\
145,810,048 \\
152,509,913 \\
233,170,111 \\
326,528,839 \\
359,216,571 \\
491,279,512 \\
281,081,514\end{array}$ & $\begin{array}{r}\text { Pounds. } \\
2,143,702 \\
3,112,528 \\
2,587,689 \\
2,894,266 \\
2,485,779 \\
10,227,702 \\
10,335,445 \\
3,494,683\end{array}$ \\
\hline
\end{tabular}

The table includes cclored and uncolored oleomargarin. No imports have been noted in reports.

The Bureau of Internal Revenue is required tokeep an accurate account of production and exportation of oleomargarin; however, the exportation figures are generally much lower than the figures reported by the Department of Commerce. 


\section{5}

\section{TABLE 58.-Quantity of CHEDDAR CHEESE in STOR- AGE warehouses on the first of each month.}

[Bureau of Markets and Crop Estimates. Thousand pounds-000 omitted.]

\begin{tabular}{|c|c|c|c|c|c|c|c|}
\hline Month. & 1915 & 1916 & 1917 & 1918 & 1919 & 1820 & 1921 \\
\hline & Pounds. & Pounds. & Pounds. & Pounds. & Pounds. & Pounds. & Pounds. \\
\hline Jan. & & 28,558 & 31,855 & & 19,823 & 53,168 & 34,115 \\
\hline & & 18,908 & 22,113 & 56,298 & 15,486 & 43,631 & 25,000 \\
\hline M & & 13,373 & 15,560 & 37,743 & 9,837 & 34,039 & 17,477 \\
\hline A & & 8,443 & 9,842 & 27,965 & 6,750 & 23,431 & 14,294 \\
\hline & & 6,546 & 7,928 & 17,736 & 6,027 & 16,963 & 13,466 \\
\hline & & 7, 301 & 11,626 & 20,395 & 12,478 & 13,502 & 17,814 \\
\hline & & 16,357 & 34,159 & 30,054 & 37,501 & 29,654 & 34,714 \\
\hline & & 31,569 & 67,595 & 48,804 & 62,645 & 51,512 & 40,642 \\
\hline Sept. & 28,575 & 46,7 & 91,5 & 55,742 & 76,661 & 60,372 & 46,706 \\
\hline Oct... & 24,144 & 49,579 & 90,6 & 42,065 & 81,359 & 55,007 & 41,842 \\
\hline Nov... & 32,428 & 45,713 & 78,08 & 33,402 & 72,889 & 48,456 & 42,969 \\
\hline Dec..... & 31,271 & 37,080 & 75,166 & 25,625 & 62,508 & 39,921 & 34,062 \\
\hline
\end{tabular}

NoTE.-Cold storage in connection with cheese in this table means the holding of cheese at a temperature of $45^{\circ} \mathrm{F}$. or less for a period of at least 30 days.

Reports began in September, 1915.

\section{TABLE 59.-PRODUCTION and EXPORTATION of FILLED CHEESE.}

[Bureau of Internal Revenue.]

\begin{tabular}{|c|c|c|c|c|c|}
\hline $\begin{array}{c}\text { Year } \\
\text { ended } \\
\text { June } 30\end{array}$ & $\begin{array}{l}\text { Produc- } \\
\text { tion. }\end{array}$ & $\begin{array}{l}\text { Exporta- } \\
\text { tion. }\end{array}$ & $\begin{array}{c}\text { Year } \\
\text { ended } \\
\text { June } 30-\end{array}$ & $\begin{array}{c}\text { Produc- } \\
\text { tion. }\end{array}$ & $\begin{array}{c}\text { Exporta- } \\
\text { tion. }\end{array}$ \\
\hline $\begin{array}{l}1897 \ldots \ldots \\
1898 \ldots \ldots \\
1899 \ldots \ldots \\
1900 \ldots \ldots \\
1901 \ldots \ldots \\
1902 \ldots .\end{array}$ & $\begin{array}{l}\text { Pounds. } \\
1,663,067 \\
1,482,861 \\
1,688,650 \\
1,574,979 \\
1,305,459\end{array}$ & $\begin{array}{r}\text { Pounds. } \\
187,113 \\
18,868 \\
1,688,650 \\
1,526,587 \\
1,253,268\end{array}$ & $\begin{array}{l}1905 \ldots \ldots \\
1906 \ldots \ldots \\
1907 \ldots \ldots \\
1908 \ldots \ldots \\
1909 \ldots \ldots \\
1910 \ldots \ldots\end{array}$ & $\begin{array}{l}\text { Pounds. } \\
\ldots . . . \\
281,429 \\
127,152 \\
137,422 \\
234,158\end{array}$ & $\begin{array}{r}\text { Pounds. } \\
281,429 \\
127,152 \\
137,422 \\
234,158\end{array}$ \\
\hline $\begin{array}{l}1903 . \ldots . \\
1904 \ldots . .\end{array}$ & $\begin{array}{l}571,183 \\
324,323\end{array}$ & $\begin{array}{l}571,183 \\
324,323\end{array}$ & $1911 \ldots$ & $33,03 i$ & 33,031 \\
\hline
\end{tabular}

1 No production reported.

2 None reported after 1912 .

A law placing a tax of 1 cent per pound on filled cheese became effective July 1, 1896: however, this kind of cheese had been made for a number of years before that. 
TABLE 60.-Average of highest daily QUOTATIONS on RENOVATED BUTTER on the New York market.

[New York Produce Review.]

\begin{tabular}{|c|c|c|c|c|c|}
\hline Year. & Price. & Year. & Price. & Year. & Price. \\
\hline $\begin{array}{l}1907 \ldots \ldots . \\
1908 \ldots \ldots \\
1909 \ldots \ldots \\
1910 \ldots \ldots \\
1911 \ldots \ldots\end{array}$ & $\begin{array}{r}\text { Cents. } \\
23.65 \\
23.09 \\
25.21 \\
26.61 \\
21.66\end{array}$ & $\begin{array}{l}1912 \ldots \ldots \\
1913 \ldots \ldots \\
1914 \ldots \ldots \\
1915 \ldots \ldots \\
1916 \ldots \ldots\end{array}$ & $\begin{array}{l}\text { Cents. } \\
26.84 \\
24.49 \\
21.14 \\
24.39 \\
29.36\end{array}$ & $\begin{array}{l}1917 \ldots \ldots . . \\
1918 \ldots \ldots \\
1919 \ldots \ldots \\
1920 \ldots \ldots\end{array}$ & $\begin{array}{r}\text { Cents. } \\
38.19 \\
43.60 \\
52.08 \\
51.54\end{array}$ \\
\hline
\end{tabular}

\section{TABLE 61.-PRODUCTION of CONDENSED and EVAPORATED MILK, by years.}

Census Bureau and Bureau of Markets and Crop Estimates- $c=$ census $\mathrm{m}=$ markets.]

\begin{tabular}{|c|c|c|c|c|c|}
\hline Year. & $\begin{array}{l}\text { Num- } \\
\text { ber of } \\
\text { fac- } \\
\text { tories. }\end{array}$ & Production. & Year. & $\begin{array}{l}\text { Num- } \\
\text { ber of } \\
\text { fac- } \\
\text { torias. }\end{array}$ & Production. \\
\hline $\begin{array}{l}1869 \mathrm{c} \ldots \ldots \\
1879 \mathrm{c} \ldots \ldots \\
1889 \mathrm{c} \ldots \ldots \\
1899 \mathrm{c} \ldots \\
1904 \mathrm{c} \ldots \\
1909 \mathrm{c} . \ldots \\
1914 \mathrm{c} \ldots \ldots\end{array}$ & $\begin{array}{r}4 \\
\cdots \\
\cdots \\
50 \\
81 \\
136 \\
250\end{array}$ & $\begin{array}{r}\text { Pounds. } \\
13,033,267 \\
37,926,821 \\
186,921,787 \\
308,485,182 \\
495,197,844 \\
875,507,438\end{array}$ & $\begin{array}{l}1916 \mathrm{~m} . . . . \\
1917 \mathrm{~m} . . . \\
1918 \mathrm{~m} . \ldots \\
1919 \mathrm{~m} \ldots . . \\
1919 \mathrm{c} \ldots \ldots \\
1920 \mathrm{~m} . \ldots .\end{array}$ & $\begin{array}{r}586 \\
596 \\
491 \\
630 \\
\ldots \ldots \\
\ldots . .\end{array}$ & $\begin{array}{r}\text { Pounds. } \\
997,835,115 \\
1,353,605,594 \\
1,675,477,360 \\
12,046,879,968 \\
2,096,973,252 \\
1,578,015,000\end{array}$ \\
\hline
\end{tabular}

1 The amounts reported for 1919 and 1920, by the Bureau of Markets and Crop Estimates, include sterilized milk canned.

\section{TABLE 62.-IMPORTS and EXPORTS of CONDENSED and EVAPORATED MILK.}

[Commerce and Navigation Reports (YB).]

\begin{tabular}{|c|c|c|c|c|c|}
\hline $\begin{array}{c}\text { Year } \\
\text { ended } \\
\text { Jun } 30-\end{array}$ & Imports. & Exports. & $\begin{array}{c}\text { Year } \\
\text { ended } \\
\text { June } 30\end{array}$ & Imports. & Exports. \\
\hline $\begin{array}{l}1892 \ldots \ldots \\
1900 \ldots . \\
1910 \ldots \\
1911 \ldots \\
1912 \ldots \\
1913 \ldots \\
1914 \ldots\end{array}$ & $\begin{array}{r}\text { Pounds. } \\
1,062,250 \\
472,958 \\
598,134 \\
630,308 \\
698,176 \\
1,778,043 \\
14,599,339\end{array}$ & $\begin{array}{c}\text { Pounds. } \\
(1) \\
(1) \\
13,311,318 \\
12,180,445 \\
20,642,738 \\
16,525,918 \\
16,209,082\end{array}$ & $\begin{array}{l}1915 \ldots . \\
1916 \ldots . \\
1917 \ldots \\
1918 \ldots \\
1919 \ldots \\
1920 \ldots \\
19202 \ldots\end{array}$ & $\begin{array}{c}\text { Pounds. } \\
33,624,189 \\
18,174,505 \\
18,375,698 \\
29,926,931 \\
20,183,723 \\
19,080,642 \\
23,755,780\end{array}$ & $\begin{array}{c}\text { Pounds. } \\
37,235,627 \\
159,577,620 \\
259,102,213 \\
529,750,032 \\
728,740,509 \\
708,463,187 \\
411,077,982\end{array}$ \\
\hline
\end{tabular}

1 Not reported.

2 Year ended December 31. 
TABLE 63.--Production of CONDENSED or EVAPORATED BUTTEŔMILK, by months, for 1918 and 1919.

[Bureau of Markets and Crop"Estimates.]

\begin{tabular}{|c|c|c|c|c|}
\hline \multirow[b]{2}{*}{ Month. } & \multicolumn{2}{|c|}{1918} & \multicolumn{2}{|c|}{1919} \\
\hline & $\begin{array}{c}\text { Facto- } \\
\text { ries } \\
\text { report- } \\
\text { ing } \\
\text { month- } \\
\text { ly pro- } \\
\text { duc- } \\
\text { tion. }\end{array}$ & $\begin{array}{l}\text { Quantity } \\
\text { made. }\end{array}$ & \begin{tabular}{|c|} 
Facto- \\
ries \\
report- \\
ing \\
month- \\
ly pro- \\
duc- \\
tion.
\end{tabular} & $\begin{array}{c}\text { Quantity } \\
\text { made. }\end{array}$ \\
\hline 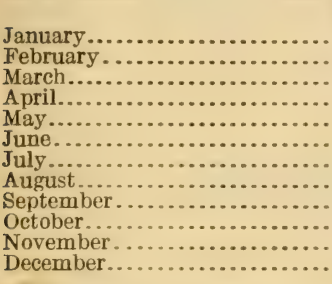 & $\begin{array}{r}17 \\
7 \\
4 \\
6 \\
7 \\
7 \\
7 \\
9 \\
12 \\
8 \\
13 \\
9\end{array}$ & $\begin{array}{r}\text { Pounds. } \\
195,356 \\
62,777 \\
101,436 \\
241,726 \\
186,146 \\
541,141 \\
562,814 \\
778,009 \\
1,201,400 \\
1,178,646 \\
966,296 \\
518,276\end{array}$ & $\begin{array}{l}11 \\
12 \\
10 \\
12 \\
17 \\
18 \\
21 \\
21 \\
21 \\
15 \\
15 \\
15\end{array}$ & $\begin{array}{r}\text { Pounds. } \\
647,078 \\
504,308 \\
524,977 \\
742,128 \\
3,348,762 \\
3,755,273 \\
3,660,376 \\
3,031,189 \\
2,424,572 \\
1,401,863 \\
1,179,880 \\
1,315,174\end{array}$ \\
\hline Total.. & $\ldots$ & $6,534,023$ & $\cdots$ & $22,535,580$ \\
\hline
\end{tabular}

Total number of factories in 1918 was 31 ; total product, $12,040,642$ pounds.

Total number of factories in 1919 was 25 ; total product, $24,282,25$ pounds.

Total number of factories in 1920 was 5: total product, $32,539,000$ pounds.

\section{TABLE 64.-Production and importation of CASEIN (lactarene).}

[Production from Census Bureau and Bureau of Markets and Crop Estimates; imports from Commerce and Navigation Reports- $\mathrm{c}=$ census; $\mathrm{m}=$ markets.]

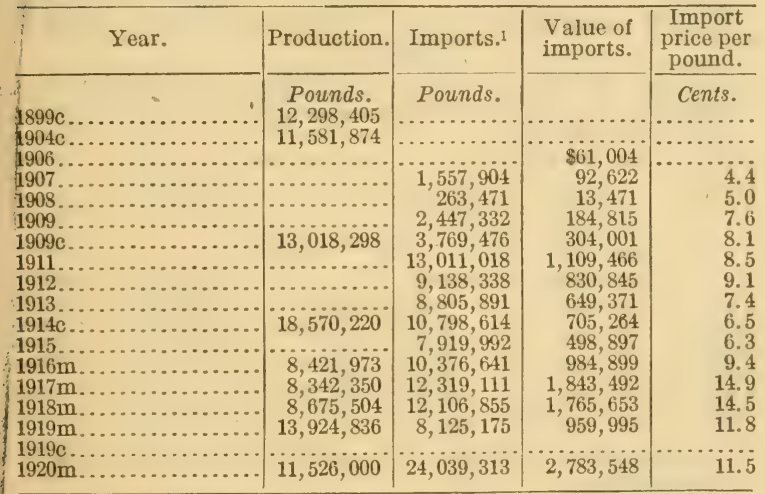

1 Fiscal year, June 30. 


\section{8}

\section{TABLE 65.-PRODUCTION of MILK POWDER, by years.}

[Bureau of Markets and Crop Estimates and Census Bureau.]

\begin{tabular}{|c|c|c|c|}
\hline Year. & $\begin{array}{c}\text { Whole-milk } \\
\text { powder. }\end{array}$ & $\begin{array}{l}\text { Skim-milk } \\
\text { powder. }\end{array}$ & Total. \\
\hline $1914 \ldots \ldots \ldots \ldots$ & $\begin{array}{l}\text { Pounds. } \\
\text { P.......... }\end{array}$ & Pounds. & \multirow{6}{*}{$\begin{array}{r}\text { Pounds. } \\
20,987,911 \\
18,586,613 \\
25,763,166 \\
30,250,701 \\
42,328,086 \\
106,711,288 \\
52,227,000\end{array}$} \\
\hline $1916 \ldots \ldots \ldots \ldots \ldots \ldots \ldots \ldots \ldots \ldots \ldots \ldots$ & $2,123,251$ & $16,463,362$ & \\
\hline $1917 \ldots \ldots \ldots \ldots \ldots \ldots \ldots \ldots \ldots \ldots \ldots \ldots \ldots \ldots \ldots \ldots$ & $3,138,809$ & $22,624,357$ & \\
\hline 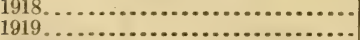 & $4,154,334$ & $25,432,341$ & \\
\hline 1919 i $191 \ldots \ldots \ldots$ & $8,660,785$ & $33,076,131$ & \\
\hline 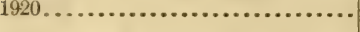 & $10,334,000$ & $41,893,000$ & \\
\hline
\end{tabular}

1 From the census reports, all kinds

\section{TABLE 66.-Production of BUTTERMILK POWDER and MALTED MILK, by years.}

[Bureau of Markets and Crop Estimates.]

\begin{tabular}{|c|c|c|}
\hline Year. & $\begin{array}{c}\text { Buttermilk } \\
\text { powder. }\end{array}$ & $\begin{array}{l}\text { Malted } \\
\text { milk. }\end{array}$ \\
\hline $\begin{array}{l}1916 \ldots \ldots \ldots \\
1917 \ldots \ldots \ldots \\
1918 \ldots \ldots \ldots \\
1919 \ldots \ldots \ldots \\
1920 \ldots \ldots\end{array}$ & $\begin{array}{r}\text { Pounds. } \\
342,485 \\
2,557,228 \\
4,341,157 \\
5,278,827 \\
5,585,906\end{array}$ & $\begin{array}{l}\text { Pounds. } \\
11,654,384 \\
13,851,939 \\
15,622,876 \\
17,435,887 \\
19,715,000\end{array}$ \\
\hline
\end{tabular}

TABLE 67.-Production and importation of MILK SUGAR (lactose).

[Production from Census Bureau and Bureau of Markets and Crop Estimates, imports from Commerce and Navigation Reports- $\mathrm{c}=$ census; $\mathrm{m}=$ markets.]

\begin{tabular}{|c|c|c|c|c|c|}
\hline Year. & $\begin{array}{c}\text { Produc- } \\
\text { tion. } 1\end{array}$ & Imports. ${ }^{2}$ & Year. & $\begin{array}{c}\text { Produc- } \\
\text { tion. }{ }^{1}\end{array}$ & Imports. ${ }^{2}$ \\
\hline $\begin{array}{l}1906 . \\
1910 . \\
1911 . \\
1912 . \\
1913 . \\
1914 \mathrm{c} .\end{array}$ & $\begin{array}{c}\text { Pounds. } \\
\ldots \\
\\
4,051,320\end{array}$ & $\begin{array}{r}\text { Pounds. } \\
4,709 \\
4,960 \\
4,732 \\
2,697 \\
9,026 \\
578,727\end{array}$ & $\begin{array}{l}1916 \mathrm{~m} . . . \\
1917 \mathrm{~m} . . . \\
1918 \mathrm{~m} . \\
1919 \mathrm{~m} . \ldots \\
1919 \mathrm{c} . \\
1920 \mathrm{~m} . \ldots\end{array}$ & \begin{tabular}{c} 
Pounds. \\
$1,844,228$ \\
$2,155,076$ \\
$3,219,461$ \\
$6,615,874$ \\
\hdashline $5,383,000$
\end{tabular} & $\begin{array}{r}\text { Pounds. } \\
22,400 \\
\text { None. } \\
\text { None. } \\
\text { None. } \\
\text { None. }\end{array}$ \\
\hline & & 250,212 & & & \\
\hline
\end{tabular}

1 Calendar year.

2 Fiscal year ended June 30. 


\section{TABLE 68.-Estimated quantity of ICE CREAM produced in the United States, by years.}

\begin{tabular}{|c|c|c|c|c|c|}
\hline Year. & $\begin{array}{l}\text { Total pro- } \\
\text { duction.1 }\end{array}$ & $\begin{array}{l}\text { Total pro- } \\
\text { duction. }{ }^{2}\end{array}$ & Year. & $\begin{array}{l}\text { Total pro- } \\
\text { duction. }{ }^{1}\end{array}$ & $\begin{array}{l}\text { Total pro- } \\
\text { duction. }\end{array}$ \\
\hline $\begin{array}{l}1909 \ldots \\
1910 \ldots \\
1911 \ldots \\
1912 \ldots \\
1913 \ldots \\
1914 \ldots \\
1915 \ldots\end{array}$ & $\begin{array}{r}\text { Gallons. } \\
80,000,000 \\
95,450,000 \\
138,000,000 \\
160,000,000 \\
172,380,000 \\
163,761,000 \\
175,224,270\end{array}$ & 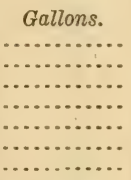 & $\begin{array}{l}1916 \ldots \\
1917 \ldots \\
1918 \ldots \\
1919 \ldots \\
1920 \ldots \\
1821 \ldots\end{array}$ & $\begin{array}{c}\text { Gallons. } \\
208,320,000 \\
219,385,000 \\
231,053,000 \\
248,381,975 \\
251,820,490 \\
216,569,212\end{array}$ & \begin{tabular}{c} 
Gallons. \\
\hdashline $210,000,000$ \\
$220,000,000$ \\
$230,000,000$ \\
$260,000,000$ \\
$\ldots \ldots \ldots . .$.
\end{tabular} \\
\hline
\end{tabular}

1 From the International Confectioner.

2 From Dairy Division, Bureau of Animal Industry, and Bureau of Markets and Crop Estimates.

\section{TABLE 69.-Production of ICE CREAM of all kinds, by} months, for the year 1920, for 2,427 factories.

[Bureau of Markets and Crop Estimates.]

\begin{tabular}{|c|c|c|c|}
\hline Month. & $\begin{array}{l}\text { Quantity } \\
\text { made. }\end{array}$ & Month. & $\begin{array}{l}\text { Quantity } \\
\text { made. }\end{array}$ \\
\hline \multirow[t]{2}{*}{ 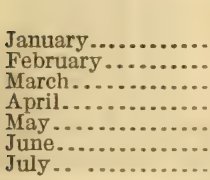 } & \multirow[t]{2}{*}{$\begin{array}{r}\text { Gallons. } \\
4,455,588 \\
4,986,374 \\
7,323,202 \\
9,296,571 \\
15,112,932 \\
20,695,601 \\
23,479,313\end{array}$} & \multirow[t]{2}{*}{ 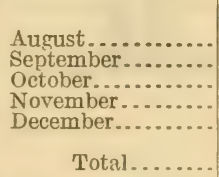 } & \begin{aligned} \multicolumn{1}{c}{ Gallons. } \\
$20,765,524 \\
15,222,342 \\
9,635,394 \\
5,279,143 \\
4,681,157\end{aligned}$ \\
\hline & & & $140,933,141$ \\
\hline
\end{tabular}

1 The reported production of ice cream is considered to be about 50 per cent of the total production. 

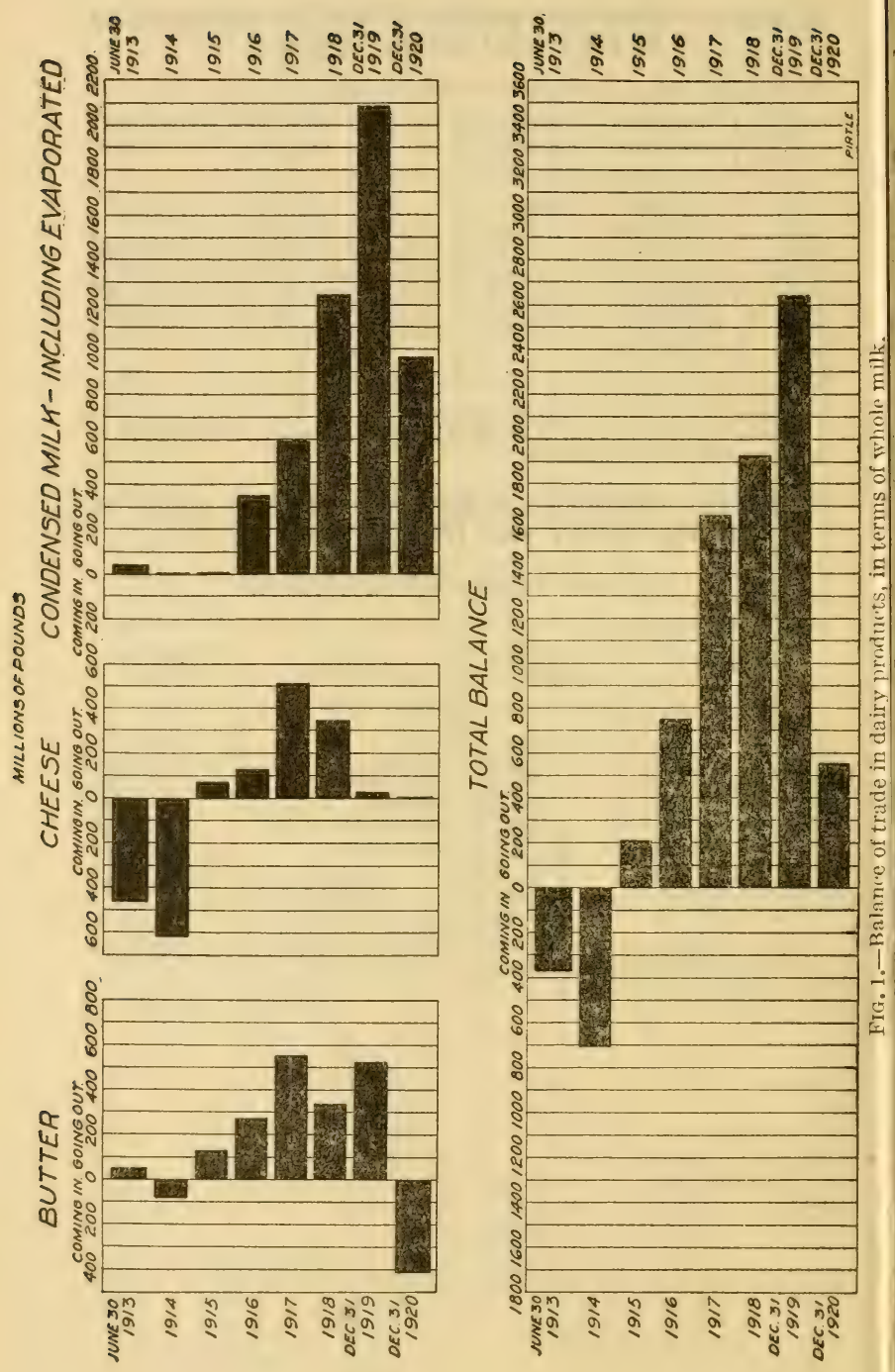


\section{TABLE 70.-Balance of BUTTER, CHEESE, and CON- DENSED MILK, taken separately.}

[From Department of Commerce reports.]

[Expressed in terms of whole milk.]

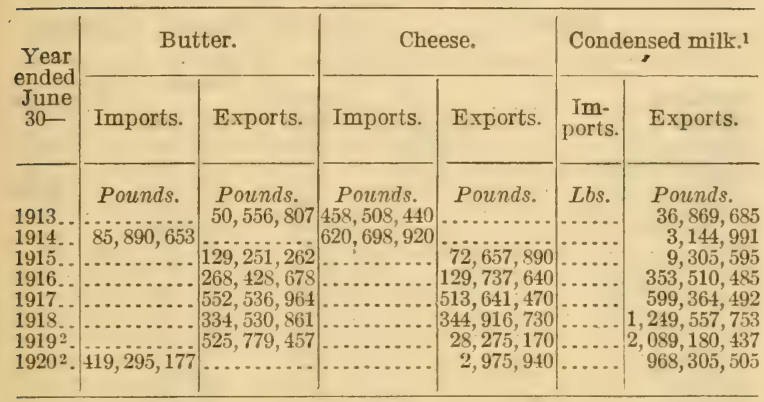

I Condensed milk includes condensed cream, condensed skim milk, and evaporated milk. The figures for 1920 are preliminary and subject to slight change.

2 Year ended Dec. 31.

\section{TABLE 71.-Total balance of BUTTER, CHEESE, and CONDENSED IVILK, combined.}

[From Department of Commerce reports.]

[In terms of whole milk.]

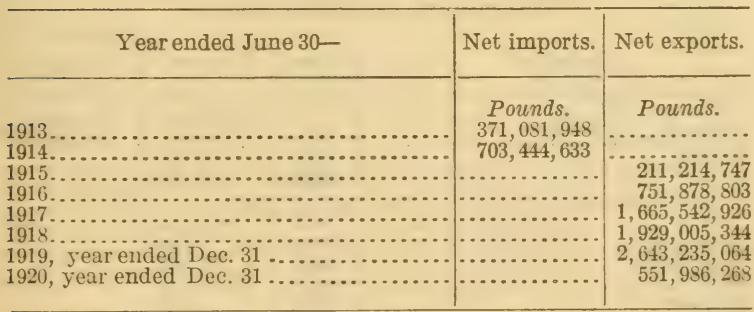

\section{Explanation of Figure $1 .^{1}$}

This chart was plotted from the difference of imports and exports, and includes butter, cheese, and condensed milk. The chart expresses these differences in terms of milk, assuming that-

1 pound of butter equals......... 21 pounds of milk.

1 pound of cheese equals......... 10 pounds of milk. 1 pound of condensed milk equals... 21/2 pounds of milk.

\footnotetext{
${ }^{1}$ For detailed figures see Tables 70 and 71.
} 
FOREIGN COUNTRIES.

TABLE 72.-Pan American countries-IMPORTS and EXPORTS of BUTTER.

[Commerce and Navigation Reports.]

\begin{tabular}{|c|c|c|c|}
\hline Country. & Year. & Imports. & Exports. \\
\hline Argentina......................... & 1919 & Pounds. & $\begin{array}{l}\text { Pounds. } \\
39,609,970\end{array}$ \\
\hline 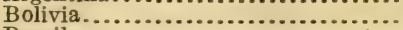 & 1913 & 114,690 & $\ldots . . . . . .$. \\
\hline Brazil.... & 1919 & 42,421 & \\
\hline British Gui & 1913 & 495,614 & \\
\hline Chile. ...... & 1915 & 314,144 & 2,844 \\
\hline Colombia 1. & 1916 & 36,909 & का \\
\hline Costa Rica.. & 1915 & 24,486 & .... \\
\hline Cuba........ & 1917 & $1,701,254$ & .......... \\
\hline Ecuador ${ }^{1} \ldots . .$. & 1916 & 975 & $\ldots \ldots$ \\
\hline ................ & 1916 & 16,883 & ........ \\
\hline ..................... & 1916 & 426,200 & ........ \\
\hline ............... & 1916 & 101,516 & ........ \\
\hline 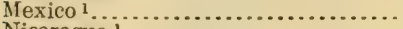 & 1916 & 667,149 & ........ \\
\hline Nicaragua 1. & 1916 & 55,756 & .... \\
\hline Panama $1 . .$. & 1916 & 621,527 & $\ldots \ldots \ldots$ \\
\hline 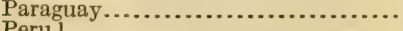 & 1915 & 9,996 & .......... \\
\hline Peru 11 . & 1916 & 50,936 & .............. \\
\hline 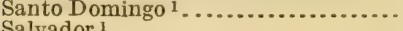 & 1916 & 56,264 & $\ldots \ldots \ldots \ldots$ \\
\hline ador $1 \ldots . . . . .$. & & $\begin{array}{r}5,533 \\
13,449\end{array}$ & $\ldots \ldots \ldots$ \\
\hline 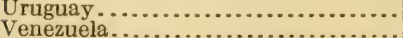 & $\begin{array}{l}1911 \\
1915\end{array}$ & $\begin{array}{r}13,440 \\
568,762\end{array}$ & ......... \\
\hline & & & \\
\hline
\end{tabular}

1 United States trade only.

\section{TABLE 73.-Pan American countries-IMPORTS and} EXPORTS of CHEESE.

[Commerce and Navigation Reports.]

\begin{tabular}{|c|c|c|c|}
\hline Country. & Year. & Imports. & Exports. \\
\hline Argentina & 1919 & Pounds. & Pounds. \\
\hline Bolivia....... & 1915 & $27,29 i$ & \\
\hline Brazil.................... & 1919 & 210,453 & (n) \\
\hline 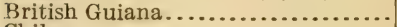 & & & \\
\hline Chile........................ & 1915 & 419,767 & 440 \\
\hline 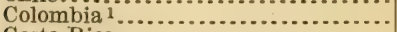 & 1916 & 15,748 & n............. \\
\hline Costa Rica & 1915 & 52,672 & ............ \\
\hline (1). & 1918 & $3,318,303$ & 5,137 \\
\hline Ecuador ${ }^{1} . . . . . . . . . . . . . . . .$. & 1916 & 1,016 & $\ldots \ldots \ldots . . . .$. \\
\hline Guatemala $1 . . . . . . . . . . . . . .$. & 1916 & 27,122 & ............. \\
\hline 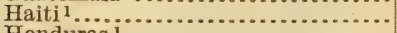 & 1916 & 52,590 & $\ldots \ldots \ldots \ldots$ \\
\hline Honduras $1 . . . . . . .$. & 1916 & 60,394 & - \\
\hline Mexico ................................ & 1913 & $1,870,385$ & $\ldots \ldots \ldots$ \\
\hline 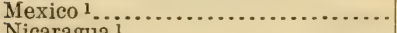 & 1916 & $\begin{array}{r}168,389 \\
27\end{array}$ & $\ldots \ldots$ \\
\hline Nicaragua $1 . . . \ldots \ldots \ldots \ldots \ldots \ldots . . . . . .$. & 1916 & $\begin{array}{r}27,460 \\
445,237\end{array}$ & $\cdots \cdots$ \\
\hline Panama $1 \ldots . . . . . . . . . . . . . . . . .$. & 1916 & $\begin{array}{r}445,237 \\
47,520\end{array}$ & $\ldots \ldots \ldots$ \\
\hline Paraguay............. & $\begin{array}{l}1915 \\
1916\end{array}$ & $\begin{array}{r}47,520 \\
5,754\end{array}$ & . \\
\hline $\begin{array}{l}\text { Peru } 1 \text {............. } \\
\text { Santo Domingo }\end{array}$ & $\begin{array}{l}1916 \\
1916\end{array}$ & $\begin{array}{r}5,754 \\
87,525\end{array}$ & $\ldots \ldots \ldots$ \\
\hline $\begin{array}{l}\text { Santo Domingo } 1 . . \\
\text { Salvador }{ }^{1} \ldots . . . . .\end{array}$ & 1916 & $\begin{array}{r}8,525 \\
5,256\end{array}$ & (............. \\
\hline Uruguay...... & 1911 & 587,216 & (1914) 28,250 \\
\hline Venezuela..... & 1915 & 102,051 & 4,010 \\
\hline
\end{tabular}

1 United States trade only. 


\section{TABLE 74.-Pan American countries-Imports of CON- DENSED MILK.}

[Commerce and Navigation Reports.]

\begin{tabular}{|c|c|c|c|c|c|}
\hline Country. & Year. & Imports. & Country. & Year., & Imports. \\
\hline Argentina & 1919 & $\begin{array}{l}\text { Pounds. } \\
\text {............. }\end{array}$ & Honduras 1 & 1916 & $\begin{array}{l}\text { Pounds. } \\
186,596\end{array}$ \\
\hline Bolivia.. & 1914 & 882,413 & Mexico ${ }^{1} \ldots$ & 1916 & $1,499,279$ \\
\hline Brazil......... & 1914 & $7,461,967$ & Nicaragua 1 & 1916 & 106,756 \\
\hline $\begin{array}{l}\text { British Gui- } \\
\text { ana......... }\end{array}$ & 1913 & & Panama $1 .$. & $\begin{array}{l}1916 \\
1915\end{array}$ & $4,071,203$ \\
\hline Chile........... & 1915 & $\ddot{1}, 573,930$ & Peru $1 . .$. & 1916 & 964.139 \\
\hline Colombia $1 . .$. & 1916 & 111,841 & Santo Do- & & \\
\hline Costa Rica... & 1915 & 245,579 & mingo ${ }^{1}$.. & 1916 & 71,945 \\
\hline Cuba........... & 1917 & $36,497,528$ & Salvador ${ }^{1}$. & 1916 & 6,720 \\
\hline Ecuador ${ }^{1} \ldots .$. & 1916 & 27,493 & Uruguay.. & 1911 & 21,504 \\
\hline Guatemala 1 . & 1916 & 86,534 & Venezuela. & 1915 & 23,911 \\
\hline Haiti ${ }^{1} . . . . . .$. & 1916 & 19,726 & & & \\
\hline
\end{tabular}

1 United States trade only, for fiscal year ended June 30.

\section{TABLE 75.-Argentina-Number of DAIRY COWS and TOTAL CATTLE.}

[Comision del Censo Agropecurio, Estadistica Agricola, and Argentine Yearbook, (YB.). $\mathrm{e}=$ census. $\mathrm{e}=$ estimates.] $^{1}$

\begin{tabular}{|c|c|c|c|c|c|}
\hline Year. & Dairy cows. & Totalcattle. & Year. & Dairy cows. & $\begin{array}{l}\text { Total } \\
\text { cattle. }\end{array}$ \\
\hline $\begin{array}{l}1888 \mathrm{c} . . \\
1895 \mathrm{c} . . \\
1908 \mathrm{c} . . \\
1914 \mathrm{c} . .\end{array}$ & $\begin{array}{l}1,800,799 \\
2,163,900 \\
2,246,000\end{array}$ & $\begin{array}{l}21,963,930 \\
21,701,526 \\
29,116,625 \\
25,866,763\end{array}$ & $\begin{array}{l}1915 \text { e... } \\
1916 \mathrm{e} . . . \\
1917 \mathrm{e} . . \\
1918 \mathrm{e} . . .\end{array}$ & $\begin{array}{l}2,291,000 \\
2,319,000 \\
2,349,000 \\
2,378,000\end{array}$ & $\begin{array}{l}26,387,000 \\
26,717,000 \\
27,052,000 \\
27,392,000\end{array}$ \\
\hline
\end{tabular}

1 Estimates by Argentine Department of Agriculture.

\section{TABLE 76.-Argentina-Number of CATTLE, by classes.}

[Report of Third Census, 1914.]

\begin{tabular}{|c|c|c|c|c|c|c|}
\hline Kinds. & $\begin{array}{l}\text { Milk } \\
\text { cows. }\end{array}$ & $\begin{array}{l}\text { Cows for } \\
\text { breeding. }\end{array}$ & Bulls. & $\begin{array}{l}\text { Calves } \\
\text { and } \\
\text { young } \\
\text { stock. }\end{array}$ & $\begin{array}{c}\text { Steers } \\
\text { and } \\
\text { oxen. }\end{array}$ & $\begin{array}{l}\text { Total } \\
\text { cattle. }\end{array}$ \\
\hline $\begin{array}{l}\text { Purebred........ } \\
\text { Mixed breed... } \\
\text { Criollos(native) }\end{array}$ & $\begin{array}{r}29,287 \\
1,263,739 \\
953,090\end{array} \mid$ & $\begin{array}{r}218,833 \\
7,785,591 \\
3,830,096\end{array}$ & $\begin{array}{r}61,846 \\
307,669 \\
308,661\end{array}$ & $\begin{array}{r}97,367 \\
6,734,650 \\
3,536,736\end{array}$ & $\begin{array}{l}333,044 \\
506,155\end{array}$ & $\begin{array}{r}407,333 \\
16,324,692 \\
9,134,738\end{array}$ \\
\hline Total... & $\mid 2,246,115$ & $11,834,520$ & 678,176 & $10,368,753$ & 739,199 & $25,866,763$ \\
\hline
\end{tabular}


TABLE 77.-Argentina-Production and consumption of BUTTER and CHEESE in factories.

[Estadistica Agricola.]

\begin{tabular}{|c|c|c|c|c|}
\hline Year. & $\begin{array}{c}\text { Butter } \\
\text { production. }\end{array}$ & $\begin{array}{c}\text { Per } \\
\text { capita } \\
\text { consump- } \\
\text { tion. }\end{array}$ & $\begin{array}{l}\text { Cheese } \\
\text { production. }\end{array}$ & $\begin{array}{c}\text { Per } \\
\text { capita } \\
\text { consump } \\
\text { tion. }\end{array}$ \\
\hline $1903 \ldots$ & $\begin{array}{l}\text { Pounds. } \\
19,477,924\end{array}$ & $\begin{array}{r}\text { Pounds. } \\
1.53\end{array}$ & $\begin{array}{l}\text { Pounds. } \\
2,619,086\end{array}$ & $\begin{array}{r}\text { Pounds. } \\
1.01\end{array}$ \\
\hline $\begin{array}{l}1904 \ldots \\
1905 \ldots\end{array}$ & $19,475,371$ & 1.35 & $4,299,897$ & i. 51 \\
\hline $1906 \ldots$ & $17,733,305$ & 1.34 & $3,073,887$ & 1.74 \\
\hline $1907 \ldots$. & $14,832,672$ & 1.31 & $4,124,411$ & 1.83 \\
\hline $1908 \ldots$ & $15,978,170$ & 1.25 & $4,135,770$ & 1.88 \\
\hline $1909 \ldots$ & $15,766,680$ & 1.02 & $6,799,630$ & 2.30 \\
\hline $1910 \ldots$ & $16,617,131$ & 1.43 & $6,044,878$ & 2.17 \\
\hline $1911 \ldots$ & $17,427,498$ & 1.92 & $7,745,993$ & 2.49 \\
\hline 1912. . & $20,849,689$ & 1.64 & $11,962,256$ & 3.07 \\
\hline $1913 \ldots$ & $22,482,506$ & 1.77 & $12,542,898$ & 2.95 \\
\hline $1914 \ldots$ & $20,518,984$ & 1.57 & $11,933,500$ & 2.50 \\
\hline $1915 .$. & $22,367,645$ & 1.47 & $14,938,370$ & 2.68 \\
\hline $1916 \ldots$ & $24,826,000$ & 1.48 & $19,195,452$ & 2.63 \\
\hline $1917 \ldots$ & $33,404,099$ & 1.41 & $35,050,935$ & 3.58 \\
\hline $1918 \ldots$ & $53,924,516$ & 1.44 & $45,009,114$ & 3.72 \\
\hline $1919 \ldots$ & ${ }^{1} 56,722,973$ & 1.42 & $149,893,528$ & 3.67 \\
\hline
\end{tabular}

1 Preliminary figures.

\section{TABLE 78.-Argentina-Imports and exports of BUTTER} and CHEESE.

[Argentine Yearbook, Estadistica Agricola.]

\begin{tabular}{|c|c|c|c|c|}
\hline \multirow{2}{*}{ Year. } & \multicolumn{2}{|c|}{ Butter. } & \multicolumn{2}{|c|}{ Cheese. } \\
\hline & Imports. & Exports. & Imports. & Exports. \\
\hline & Pounds. & Pounds. & Pounds. & Pounds. \\
\hline $\begin{array}{l}1870 \ldots \\
1880 \ldots\end{array}$ & 103,001 & & $1,263,317$ & ……... \\
\hline $\begin{array}{l}1880 \ldots \\
1890 \ldots\end{array}$ & $\begin{array}{r}240,777 \\
\ldots \ldots \ldots\end{array}$ & $\begin{array}{r}8,721 \\
\ldots \ldots \ldots \ldots\end{array}$ & & 3,104 \\
\hline $1900 \ldots$ & $\ldots .$. & & $3,028,728$ & 1,887 \\
\hline $1910 \ldots$ & 1,375 & $6,341,589$ & $9,536,041$ & 809 \\
\hline $1911 \ldots$ & 150,175 & $3,077,652$ & $10,845,500$ & 1,142 \\
\hline $1912 \ldots$ & & $8,105,747$ & $11,848,927$ & 4,281 \\
\hline $1913 \ldots$ & .............. & $8,342,340$ & $11,122,295$ & 16,186 \\
\hline $1914 .$. & ......... & $7,676,417$ & $8,453,245$ & 7,771 \\
\hline $1915 .$. & 798 & $10,191,866$ & न',305,954 & 13,344 \\
\hline $1916 \ldots$ & 705 & $12,502,286$ & $3,133,279$ & 502,648 \\
\hline 1917... & 805 & $21,671,558$ & 689,356 & $6,014,148$ \\
\hline $1918 .$. & 249 & $41,821,262$ & 81,894 & $14,177,253$ \\
\hline $1919 \ldots$ & 9,921 & $44,863,610$ & 209,243 & $19,561,969$ \\
\hline $1920 \ldots$ & & $141,249,486$ & & $113,860,915$ \\
\hline
\end{tabular}

1 Special report to Bureau of Markets and Crop Estimates. 


\section{5}

\section{TABLE 79-Australia-Production of DAIRY PRODUCTS.}

[Yearbook and Bulletin of Production.]

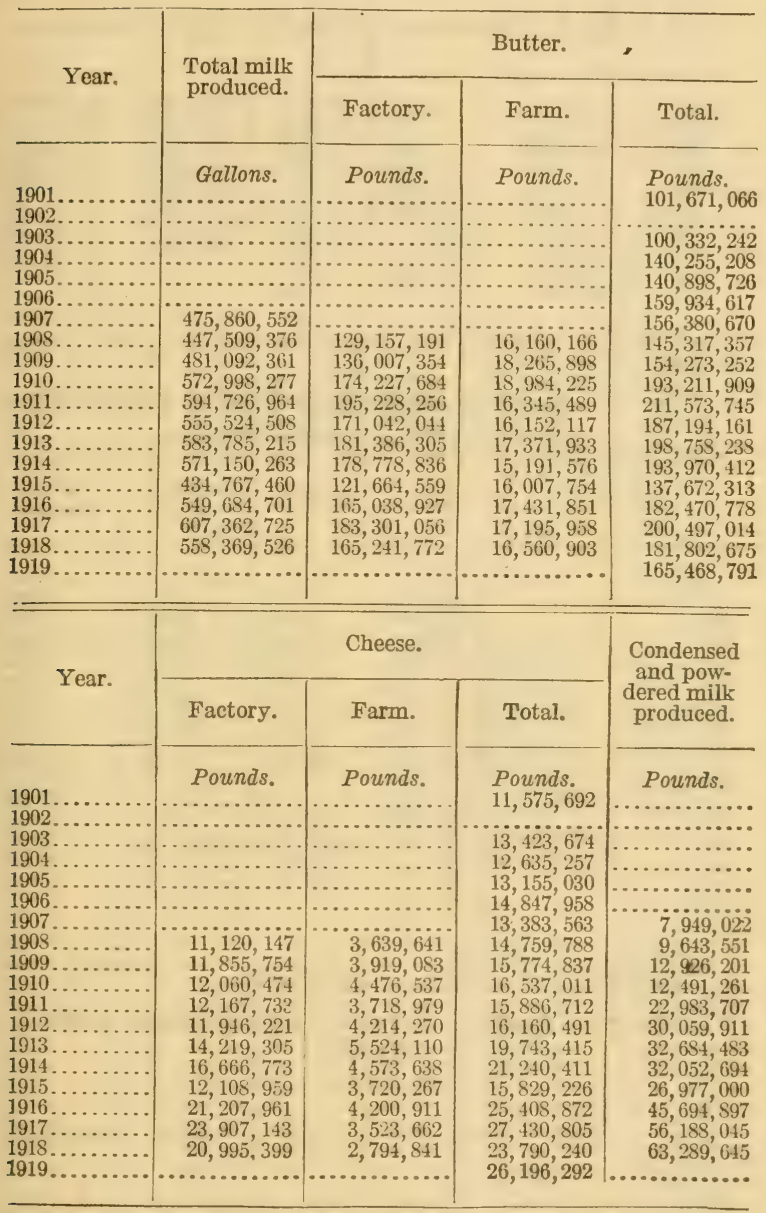




\section{TABLE 80.-Australia-Number of DAIRY COWS and TOTAL CATTLE.}

[Statistical account by T. A. Coughlin. Also An. Rep., U. S. Bureau of Animal Industry, 1900 (YB.)].

\begin{tabular}{|c|c|c|c|c|c|}
\hline Year. & $\begin{array}{l}\text { Dairy } \\
\text { cows. }\end{array}$ & Total cattle. & Year. & $\begin{array}{l}\text { Dairy } \\
\text { cows. }\end{array}$ & Total cattle. \\
\hline 1788 & & (1) 6 & 1911 & $2,120,469$ & $11,828,954$ \\
\hline 1792 & & 23 & 1912 & $2,056,143$ & $11,577,259$ \\
\hline 1800 & & 1,044 & 1913 & $2,035,717$ & $11,483,882$ \\
\hline 1851 & & $1,921,963$ & 1914 & $1,938,834$ & $11,047,677$ \\
\hline 1861 & & $4,039,839$ & 1915 & $1,684,393$ & $9,931,416$ \\
\hline 1871 & & $4,713,820$ & 1916 & $1,747,351$ & $10,467,737$ \\
\hline 1881 & & $8,709,628$ & 1917 & $1,904,906$ & $11,956,024$ \\
\hline 1891 & & $11,112,112$ & 1918 & $1,902,036$ & $12,738,852$ \\
\hline 1900 & $21,143,276$ & $8,640,225$ & 1919 & ............. & $12,888,617$ \\
\hline 1910 & $2,064,599$ & $11,744,714$ & 1920 & & \\
\hline
\end{tabular}

1 The first importation of cattle was in 1788 and consisted of six head. 21899.

\section{TABLE 81.-Australia-Exports of DAIRY PRODUCTS.}

[Trade Customs and Excise Revenue.]

\begin{tabular}{|c|c|c|c|c|}
\hline Year. & Butter. & Cheese. & $\begin{array}{l}\text { Condensed } \\
\text { milk. }\end{array}$ & $\begin{array}{c}\text { Milk } \\
\text { powder. }\end{array}$ \\
\hline $\begin{array}{l}1899 \ldots \ldots \\
1890 \ldots \ldots \\
1899 \ldots \ldots \\
1901 \ldots \ldots \\
1905 \\
1910 \\
1911 \\
19112 \ldots \\
1912 \ldots \ldots\end{array}$ & \begin{tabular}{r} 
Pounds. \\
$2,363,088$ \\
$2,976,848$ \\
$13,608,224$ \\
$34,536,277$ \\
$55,904,151$ \\
$87,894,943$ \\
$101,722,136$ \\
$66,679,120$ \\
$75,795,642$ \\
\hdashline $54,021,523$ \\
$16,722,010$ \\
$75,840,568$ \\
$72,277,526$ \\
$41,114,764$ \\
$92,420,800$
\end{tabular} & $\begin{array}{r}\text { Pounds. } \\
\text { None. } \\
\text { None. } \\
\text { None. } \\
293,381 \\
193,608 \\
911,760 \\
1,149,134 \\
146,022 \\
1,602,609 \\
\ldots 2,542,478 \\
128,229 \\
10,569,279 \\
8,426,647 \\
2,303,276 \\
\ldots \ldots \ldots . . .\end{array}$ & 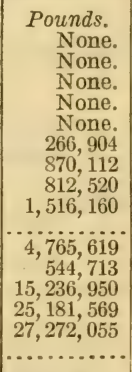 & $\begin{array}{r}\text { Pounds. } \\
\text { None. } \\
\text { None. } \\
\text { None. } \\
\text { None. } \\
\text { None. } \\
\text { None. } \\
146,960 \\
833,894 \\
1,263,803 \\
141,777 \\
163,930 \\
\text { None. } \\
404,823 \\
662,943 \\
\ldots . . . . . .\end{array}$ \\
\hline
\end{tabular}

1 Calendar year from 1899 to 1913, inclusive; then changed to fiscal year ended June 30 . No figures for the year 1914.

\section{TABLE 82.-Austria-Number of COWS and TOTAL CATTLE.}

[Various authorities; (YB.).]

\begin{tabular}{|c|c|c|c|c|c|}
\hline Year. & Cows. & Total cattle. & Year. & Cows. & Total cattle. \\
\hline $\begin{array}{l}1869 \ldots \\
1880 \ldots \\
1890 \ldots\end{array}$ & $\begin{array}{l}3,831,136 \\
4,138,625 \\
4,254,303\end{array}$ & $\begin{array}{l}7,425,212 \\
8,584,077 \\
8,643,936\end{array}$ & $\begin{array}{l}1900 \ldots . . . \\
1910 \ldots .\end{array}$ & $\begin{array}{l}4,749,152 \\
4.901,886\end{array}$ & $\begin{array}{l}9,511,170 \\
9,160,009\end{array}$ \\
\hline
\end{tabular}




\section{TABLE 83.-Canada-Number of DAIRY COWS and - TOTAL CATTLE.}

[The Dairy Industry of Canada; and Montkly Bulletin of Agricultural Statistics.]

\begin{tabular}{|c|c|c|c|c|c|}
\hline Year. ${ }^{1}$ & Dairy cows. & $\begin{array}{l}\text { Total cattle } \\
\text { (YB.). }\end{array}$ & Year. ${ }^{1}$ & Dairy cows. & $\begin{array}{l}\text { Total cattle } \\
\text { (YB.). }\end{array}$ \\
\hline $1871^{2} \ldots$ & $1,251,209$ & $2,624,290$ & 1914. & $2,673,286$ & $6,036,817$ \\
\hline $1881 \ldots$ & $1,595,800$ & $3,514,989$ & 1915. & $2,666,846$ & $6,066,001$ \\
\hline 1891. & 1857,112 & $4,120,586$ & 1916. & $2,833,433$ & $6,594,151$ \\
\hline 1901. & $2,408,677$ & $5,327,504$ & 1917. & $3,202,283$ & $7,920,940$ \\
\hline 1910 . & $2,853,957$ & $7,104,893$ & 1918. & $3,538,600$ & $10,045,867$ \\
\hline 1911. & $2,594,179$ & $6,533,436$ & 1919. & $3,548,437$ & $10,085,011$ \\
\hline 1912. & $2,604,488$ & $6,431,861$ & 1920 . & $3,530,238$ & $9,477,380$ \\
\hline 1913. & $2,740,434$ & $6,656,121$ & $1921 \ldots$ & $3,736,832$ & $10,206,205$ \\
\hline
\end{tabular}

1 On June 15.

2 Data for 1871 included only Ontario, Quebec, New Brunswick, and Nova Scotia.

\section{TABLE 84.-Canada--Production of BUTTER and CHEESE.}

[Dairy and Cold Storage Commissioner's Reports, and Yearbooks.]

\begin{tabular}{|c|c|c|c|c|}
\hline \multirow{2}{*}{ Year. } & \multicolumn{2}{|c|}{ Farm. } & \multicolumn{2}{|c|}{ Factory. } \\
\hline & Butter. & Cheese. & Butter. & Cheese. \\
\hline 1871.. & $\begin{array}{l}\text { Pounds. } \\
74,190,584\end{array}$ & $\begin{array}{l}\text { Pounds. } \\
4,984,843\end{array}$ & Pounds. & Pounds. \\
\hline $1881 \ldots$ & $102,545,169$ & $3,184,996$ & & $\cdots$ \\
\hline $1891 \ldots$ & $111,577,210$ & $6,267,203$ & & \\
\hline $1901 \ldots \ldots \ldots$ & $105,343,076$ & ………… & $36,066,739$ & $220,833,269$ \\
\hline $1911 \ldots$ & $137,110,200$ & $1,371,092$ & $64,698,165$ & $199,904,205$ \\
\hline 1915. & ..................... & $\ldots \ldots \ldots . . . . . .$. & $83,991,453$ & $183,887,837$ \\
\hline $1916 \ldots \ldots \ldots$ & $\ldots \ldots \ldots$ & ............. & $82,564,130$ & $192,968,597$ \\
\hline 1917. & $\ldots \ldots$ & . & $87,526,939$ & $194,904,336$ \\
\hline $1918 \ldots$ & $\because \because x^{\prime}$ & $\ldots \ldots \ldots$ & $93,266,876$ & $174,881,957$ \\
\hline $\begin{array}{l}1919 \ldots \\
1920 \ldots\end{array}$ & $1125,000,000$ & 月. & $101,554,131$ & $167,734,982$ \\
\hline & $125,000,000$ & & $111,691,718$ & $149,201,856$ \\
\hline
\end{tabular}

\begin{tabular}{|c|c|c|}
\hline \multirow{2}{*}{ Year. } & \multicolumn{2}{|c|}{ Total. } \\
\hline & Butter. & Cheese. \\
\hline $1871 \ldots \ldots \ldots \ldots \ldots \ldots$ & $\begin{array}{l}\text { Pounds. } \\
74,190,584\end{array}$ & $\begin{array}{l}\text { Pounds. } \\
23,000,000\end{array}$ \\
\hline 1881 & $\ldots \ldots . . . . . . . .$. & $61,000,000$ \\
\hline 1891. & (3) & $109,000,000$ \\
\hline 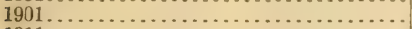 & $141,409,815$ & \\
\hline $1911 \ldots \ldots$ & $201,808,365$ & $201,275,297$ \\
\hline 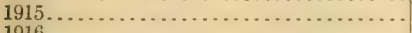 & ................. & (................. \\
\hline 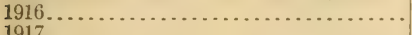 & (n....... & (n............. \\
\hline 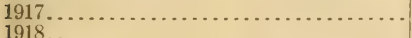 & $\cdots \ldots \ldots \ldots \ldots \ldots$ & (n. \\
\hline 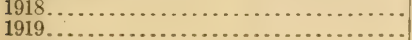 & $226,554,13 i$ & $167,734,982$ \\
\hline 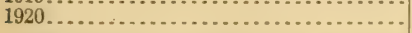 & $236,691,718$ & $149,201,856$ \\
\hline
\end{tabular}

1 Farm butter estimated by Canadian officials. 
TABLE 85.-Canada-Imports and exports of BUTTER and CHEESE.

[Trade and Commerce Reports and Bulletin Commercial Series No. 1.]

\begin{tabular}{|c|c|c|c|c|}
\hline \multirow{2}{*}{ Fiscal year. } & \multicolumn{2}{|c|}{ Butter. } & \multicolumn{2}{|c|}{ Cheese. } \\
\hline & Imports. & Exports. & Imports. & Exports. \\
\hline 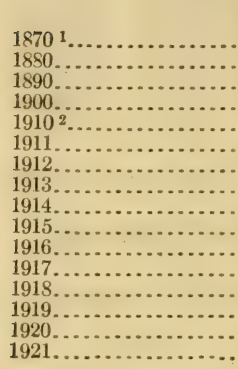 & $\begin{array}{r}\text { Pounds. } \\
83,728 \\
126,848 \\
381,329 \\
1,175,301 \\
393,582 \\
1,227,390 \\
3,874,587 \\
7,989,269 \\
7,317,259 \\
6,822,540 \\
4,309,831 \\
997,335 \\
434,049 \\
1,934,062 \\
397,955 \\
3,741,628\end{array}$ & $\begin{array}{r}\text { Pounds. } \\
12,260,887 \\
18,535,362 \\
1,951,585 \\
25,259,737 \\
4,615,380 \\
3,142,682 \\
8,844,402 \\
828,323 \\
1,228,753 \\
2,724,913 \\
3,441,183 \\
7,990,435 \\
4,926,154 \\
13,659,157 \\
17,612,605 \\
9,739,414\end{array}$ & $\begin{array}{r}\text { Pounds. } \\
59,744 \\
106,815 \\
127,556 \\
301,098 \\
683,778 \\
866,653 \\
919,189 \\
1,495,758 \\
1,512,108 \\
1,162,465 \\
971,821 \\
785,221 \\
343,269 \\
172,943 \\
362,693 \\
551,040\end{array}$ & \begin{tabular}{|c|}
\multicolumn{1}{|c}{ Pounds. } \\
$5,8277,82$ \\
$40,368,678$ \\
$91,260,187$ \\
$185,984,430$ \\
$180,859,886$ \\
$181,893,724$ \\
$163,450,684$ \\
$155,216,392$ \\
$144,478,340$ \\
$137,601,661$ \\
$168,961,583$ \\
$180,733,426$ \\
$169,530,753$ \\
$152,207,037$ \\
$126,395,777$ \\
$133,620,340$
\end{tabular} \\
\hline
\end{tabular}

11870 to 1900 , year ended June 30 .

21910 to 1920, year ended Mar. 31.

\section{TABLE 86.-Canada-Exports of CREAM, CASEIN, and CONDENSED and EVAPORATED MILK.}

[Trade and Commerce Reports, and Bulletin Commercial Series N o. 1.]

\begin{tabular}{|c|c|c|c|}
\hline Fiscal year. & Cream. & Casein. & $\begin{array}{l}\text { Condensed } \\
\text { and evap- } \\
\text { orated milk. }\end{array}$ \\
\hline $\begin{array}{l}1911 \\
1912 \ldots \ldots \\
1913 \\
1914 \\
1915 \\
1916 \\
1916 \\
1917 \\
1918 \\
1919 \\
1919 \\
1920 \\
1921\end{array}$ & $\begin{array}{r}\text { Gallons. } \\
1,823,821 \\
886,266 \\
820,360 \\
1,323,929 \\
1,895,575 \\
1,262,280 \\
803,498 \\
585,601 \\
485,015 \\
795,780 \\
1,279,195\end{array}$ & $\begin{array}{r}\text { Pounds. } \\
1,515,172 \\
1,021,197 \\
349,865 \\
270,486 \\
230,045 \\
50,564 \\
50,588 \\
16,200 \\
9,110 \\
\end{array}$ & $\begin{array}{r}\text { Pounds. } \\
6,584,828 \\
4,389,350 \\
335,849 \\
9,339,382 \\
18,355,975 \\
13,247,834 \\
15,858,622 \\
43,656,718 \\
50,786,856 \\
54,247,498 \\
49,147,541\end{array}$ \\
\hline
\end{tabular}

Year ended Mar 31. 
TABLE 87.-Canada-Production of CONDENSED and POWDERED MILK.

[Canadian News Letter, and Dairy and Cold Storage Commissioner's Reports.]

\begin{tabular}{|c|c|c|c|c|c|}
\hline Year. & $\begin{array}{l}\text { Condensed } \\
\text { milk. }\end{array}$ & $\begin{array}{c}\text { Milk } \\
\text { powder. }\end{array}$ & Year. & $\begin{array}{l}\text { Condensed } \\
\text { milk. }\end{array}$ & $\begin{array}{c}\text { Milk } \\
\text { powder. }\end{array}$ \\
\hline $\begin{array}{l}1910 \ldots \ldots \\
1917 \ldots \ldots \\
1918 \ldots . . .\end{array}$ & $\begin{array}{c}\text { Pounds. } \\
27,831,596 \\
61,520,811 \\
79,807,971\end{array}$ & $\begin{array}{l}\text { Pounds. } \\
5,323,537 \\
3,979,514 \\
5,530,915\end{array}$ & $\begin{array}{l}1919 \ldots \\
1920 \ldots\end{array}$ & $\begin{array}{c}\text { Pounds. } \\
85,961,610 \\
92,104,562\end{array}$ & $\begin{array}{l}\text { Pounds. } \\
6,591,099 \\
7,574,668\end{array}$ \\
\hline
\end{tabular}

\section{TABLE 88.-Denmark-Number of DAIRY COWS and TOTAL CATTLE.}

[Department of Statistics of Denmark, Statistisk Aarbog, and Statistik Arsbok.]

\begin{tabular}{|c|c|c|c|c|c|}
\hline Year. & $\begin{array}{l}\text { Dairy } \\
\text { cows. }\end{array}$ & $\begin{array}{c}\text { Total } \\
\text { cattle } \\
\text { (YB.). }\end{array}$ & Year. & $\begin{array}{l}\text { Dairy } \\
\text { cows. }\end{array}$ & $\begin{array}{c}\text { Total } \\
\text { cattle } \\
\text { (YB.). }\end{array}$ \\
\hline $\begin{array}{l}1876 \ldots \ldots \\
1881 \ldots \ldots \\
1893 \ldots \ldots \\
19031 \ldots . \\
19091 \ldots \\
19141 \ldots \\
19152 \ldots\end{array}$ & $\begin{array}{r}898,012 \\
898,790 \\
1,011,098 \\
1,089,073 \\
1,281,974 \\
1.310,268 \\
1,281,132\end{array}$ & $\begin{array}{r}1,348,321 \\
1,696,190 \\
1,840,466 \\
2,253,982 \\
2,462,862 \\
2,416,471\end{array}$ & $\begin{array}{l}1916^{3} \ldots \ldots \\
19177^{4} \ldots \\
1918^{5} \ldots \\
1919^{5} \ldots \\
1920 \\
19215\end{array}$ & $\begin{array}{l}1,141,246 \\
1,147,183 \\
1,024,417 \\
1,042,143 \\
1,195,733 \\
1,184,268\end{array}$ & $\begin{array}{l}2,289,996 \\
2,458,158 \\
2,123,722 \\
2,188,142 \\
2,286,408 \\
2,591,000\end{array}$ \\
\hline
\end{tabular}

1 Number on July 15.

2 Number on May 15.

3 Number on Feb. 20.
4 Number on July 1.

5 Number on July 15 , including South Jutland.

TABLE 89.-Denmark-Imports and exports of BUTTER. [Denmarks Vareindforsel og udforsel; Statistisk Tabelvaerk. (YB.)].

\begin{tabular}{|c|c|c|}
\hline \multirow{2}{*}{ Year. } & \multicolumn{2}{|c|}{ Butter. } \\
\hline & Imports. & Exports. \\
\hline $\begin{array}{l}1866 \ldots . . \\
1870 \ldots . \\
1880 \ldots . \\
1890 \ldots . \\
1900 \ldots . \\
1910 \ldots \\
1911 \ldots . . \\
1912 \ldots . \\
1913 \ldots\end{array}$ & \begin{tabular}{|c}
$\begin{array}{c}\text { Pounds. } \\
\text { Not given. }\end{array}$ \\
$\ldots . . . . . . . .$. \\
\hdashline$\ldots .$. \\
$120,549,573$ \\
$146,700,515$ \\
$6,240,561$ \\
$6,026,935$ \\
$5,966,000$ \\
$6,242,104$
\end{tabular} & $\begin{array}{r}\text { Pounds. } \\
10,710,194 \\
13,804,782 \\
27,057,726 \\
98,167,019 \\
131,100,071 \\
195,052,426 \\
197,481,675 \\
187,755,000 \\
200,670,408\end{array}$ \\
\hline
\end{tabular}

\begin{tabular}{|c|c|c|}
\hline \multirow{2}{*}{ Year. } & \multicolumn{2}{|c|}{ Butter. } \\
\hline & Imports. & Exports. \\
\hline $\begin{array}{l}1914 \ldots \\
1915 \ldots . \\
1916 \ldots \\
1917 \ldots . \\
1918 \ldots \\
1919 \ldots . \\
1920 \ldots \\
1921 \ldots\end{array}$ & $\begin{array}{r}\text { Pounds. } \\
3,054,473 \\
686,733 \\
190,698 \\
882 \\
220\end{array}$ & $\begin{array}{r}\text { Pounds. } \\
210,084,491 \\
223,964,432 \\
211,090,450 \\
135,501,550 \\
32,305,547 \\
80,908,820 \\
2165,345,000 \\
2199,584,863\end{array}$ \\
\hline
\end{tabular}

1 Imports for 1890 and 1900 were "general." From 1910 the imports were for "special trade only."

2 Semiofticial, preliminary. 


\section{TABLE 90.-Denmark-Average of the official weekly quotations on BUTTER.}

[Sm $\phi r$ Tidende.]

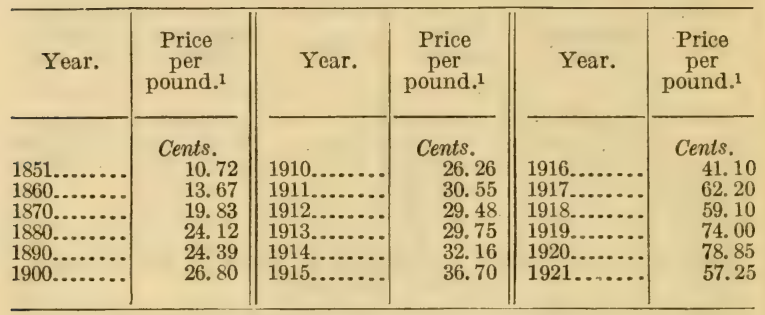

1 Conversion made at the rate of 1 krone equals 26.8 cents.

\section{TABLE 91.-Finland-Number of COWS, TOTAL CAT- TLE, and REINDEER.}

[Statistisk Arsbok.]

\begin{tabular}{|c|c|c|c|c|c|c|c|}
\hline Year. & Cows. & $\begin{array}{l}\text { Total cat- } \\
\text { tle (YB.). }\end{array}$ & $\begin{array}{l}\text { Rein- } \\
\text { deer. }\end{array}$ & Year. & Cows. & $\begin{array}{l}\text { Total cat- } \\
\text { tle (YB.). }\end{array}$ & $\begin{array}{l}\text { Rein- } \\
\text { deer. }^{3}\end{array}$ \\
\hline $\begin{array}{l}1865 . . \\
1870 . \\
1880 . . \\
1890 . . \\
1900 . . \\
1910^{1} \\
1911 . .\end{array}$ & $\begin{array}{r}670,897 \\
687,060 \\
795,575 \\
928,276 \\
1,072,002 \\
1,142,908 \\
1,150,500\end{array}$ & $\begin{array}{r}954,321 \\
997,960 \\
1,132,002 \\
1,305,187 \\
1,427,560 \\
1,573,163 \\
\ldots \ldots \ldots \ldots\end{array}$ & 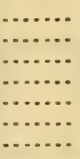 & $\begin{array}{l}1912 . . \\
1913 . . \\
1914 . \\
19152 . \\
1916 . \\
1917 . \\
1918 .\end{array}$ & $\begin{array}{l}1,151,659 \\
1,141,757 \\
1,164,229 \\
1,114,447 \\
1,080,558 \\
1,075,749 \\
1,015,901\end{array}$ & \begin{tabular}{r} 
\\
\hdashline $1,603,720$ \\
$1,537,936$ \\
$1,501,975$ \\
$1,500,670$ \\
$1,438,573$
\end{tabular} & \\
\hline
\end{tabular}

1 The figures for 1910 are for month of September.

2 Data for 1915 to 1918 are directly from the Finnish Government.

3 The number of reindeer since 1895 has averaged about 125,000 head. 


\section{TABLE 92.-France-Number of COWS, TOTAL CAT- TEE, SHEEP, and GOATS.}

[Statistique Agricole Annuelle, and Annuaire Statistique; also Enquête sur l'Industrie de Lait, 1902; (YB.).]

\begin{tabular}{|c|c|c|c|c|}
\hline Year & Cows. & $\begin{array}{l}\text { Total } \\
\text { cattle. }\end{array}$ & Sheep. & Goats. \\
\hline $840 \ldots$ & $\begin{array}{l}5,501,825 \\
5,781,465 \\
6,408,261 \\
6,587,639 \\
6,509,325 \\
6,673,460 \\
7,819,582 \\
7,596,250 \\
7,606,670 \\
7,745,750 \\
7,794,270 \\
6,663,355 \\
6,266,035 \\
6,221,850 \\
6,177,940 \\
6,237,470 \\
6,327,510 \\
6,544,460\end{array}$ & $\begin{array}{l}11,761,538 \\
11,970,737 \\
12,811,589 \\
12,997,054 \\
13,662,585 \\
13,708,997 \\
14,520,832 \\
14,532,030 \\
14,435,530 \\
14,705,900 \\
14,787,410 \\
12,668,000 \\
12,514,414 \\
12,342,000 \\
12,241,980 \\
12,250,820 \\
12,373,660 \\
12,755,720\end{array}$ & $\begin{array}{r}14,804,946 \\
14,572,430 \\
9,758,528 \\
\ldots 8,804,401 \\
20,180,000 \\
17,111,000 \\
16,425,000 \\
16,468,000 \\
16,213,000 \\
14,038,000 \\
13,483,189 \\
12,379,000 \\
10,587,000 \\
9,496,000 \\
8,991,000 \\
9,376,630\end{array}$ & \begin{tabular}{r}
$1,216,774$ \\
$2,336,332$ \\
\hdashline $1,335,736$ \\
$1,355,000$ \\
$1,411,000$ \\
$1,424,000$ \\
$1,409,000$ \\
$1,453,000$ \\
$1,317,000$ \\
$1,230,000$ \\
$1,177,000$ \\
\hdashline $1,197,000$ \\
$1,167,000$
\end{tabular} \\
\hline
\end{tabular}

1 Not including the invaded portion.

\section{TABLE 93.-France-IMPORTS of BUTTER, CHEESE, and CONDENSED MILK.}

[Tableau Général du Commerse de la France; (YB.).]

\begin{tabular}{|c|c|c|c|}
\hline Year. & Butter. & Cheese. & $\begin{array}{l}\text { Condensed } \\
\text { milk. }\end{array}$ \\
\hline $\begin{array}{l}1850 \\
1860 \\
1870 \\
1880 \\
1890 \\
1900 \\
1910 \\
1911 \\
1912 \\
1912 \\
1913 \\
1914 \\
1915 \\
1916 \\
1917 \\
1918 \\
1918 \\
1919 \\
1920\end{array}$ & $\begin{array}{r}\text { Pound.s. } \\
3,493,996 \\
3,926,503 \\
6,635,326 \\
15,641,716 \\
14,164,738 \\
13,559,262 \\
10,656,375 \\
19,939,064 \\
14,179,326 \\
13,033,816 \\
13,654,631 \\
1,710,549 \\
625,004 \\
742,288 \\
1,983,693 \\
12,752,288 \\
4,812,000\end{array}$ & $\begin{array}{r}\text { Pounds. } \\
9,568,652 \\
11,493,484 \\
23,756,057 \\
34,811,710 \\
28,725,722 \\
42,635,400 \\
49,011,340 \\
49,423,380 \\
55,494,410 \\
52,085,000 \\
45,521,242 \\
46,743,913 \\
24,139,268 \\
12,046,816 \\
11,206,202 \\
15,233,243 \\
15,130,000\end{array}$ & 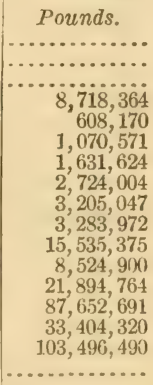 \\
\hline
\end{tabular}




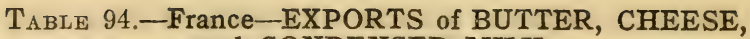
and CONDENSED MILK.

[Tableau Général du Commerce de la France; (YB.).]

\begin{tabular}{|c|c|c|c|}
\hline Year. & Butter. & Cheese. & $\begin{array}{l}\text { Condensed } \\
\text { milk. }\end{array}$ \\
\hline $\begin{array}{l}1850 \ldots \\
1860 \\
1870 \\
1880 \\
1890 \\
1900 \\
1910 \\
1911 \\
1911 \\
1912 \\
1913 \\
1914 \\
1914 \\
1915 \\
1916 \\
1917 \\
1917 \\
1918 \\
1919 \\
1920\end{array}$ & $\begin{array}{r}\text { Pounds. } \\
4,870,497 \\
26,073,317 \\
42,300,974 \\
68,484,843 \\
89,784,546 \\
44,658,761 \\
48,427,787 \\
28,221,305 \\
37,571,675 \\
38,359,820 \\
44,619,000 \\
50,336,088 \\
18,936,632 \\
6,728,439 \\
2,619,506 \\
1,119,055 \\
4,812,000\end{array}$ & $\begin{array}{r}\text { Pounds. } \\
1,866,048 \\
3,458,969 \\
4,676,261 \\
9,407,683 \\
12,232,137 \\
18,467,555 \\
25,160,659 \\
24,041,383 \\
27,689,555 \\
37,386,709 \\
22,323,780 \\
16,241,729 \\
11,704,221 \\
7,403,488 \\
5,212,556 \\
7,336,027 \\
15,130,000\end{array}$ & 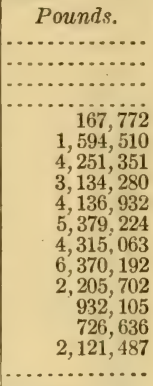 \\
\hline
\end{tabular}

TABLE 95.-Germany-Number of COWS, TOTAL CATTLE, and GOATS.

[Statistisches Jahrbuch, and Imperial Gazette.]

\begin{tabular}{|c|c|c|c|}
\hline Year. & Cows. & $\begin{array}{c}\text { Total cattle } \\
\text { (YB.). }\end{array}$ & Goats(YB.). \\
\hline 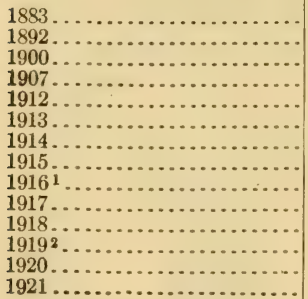 & 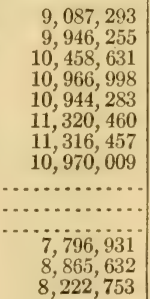 & $\begin{array}{l}15,786,764 \\
17,555,834 \\
18,939,692 \\
20,630,544 \\
20,182,021 \\
20,994,344 \\
21,817,375 \\
20,316,948 \\
20,338,950 \\
19,649,575 \\
17,226,855 \\
16,317,329 \\
16,789,844 \\
16,839,559\end{array}$ & $\begin{array}{r}3,091,508 \\
3,266,997 \\
3,330,000 \\
3,410,396 \\
3,548,384 \\
3,538,414 \\
3,438,296 \\
\ldots . \ldots . \ldots \\
4,564,210\end{array}$ \\
\hline
\end{tabular}

1 September.

\section{TABLE 96.-Germany-Per capita BUTTER and CHEESE CONSUMPTION.}

\begin{tabular}{|c|c|c|c|}
\hline & Year. & $\begin{array}{l}\text { Butter } \\
\text { consump- } \\
\text { tion. }\end{array}$ & $\begin{array}{c}\text { Cheese } \\
\text { consump- } \\
\text { tion. }\end{array}$ \\
\hline $\begin{array}{l}1912 . \\
1913 . \\
1918 .\end{array}$ & 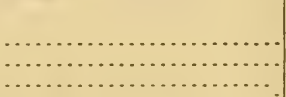 & $\begin{array}{r}\text { Pounds. } \\
14.7 \\
9.2\end{array}$ & \begin{tabular}{r}
\multicolumn{2}{r}{ Pounds. } \\
9.5 \\
$\ldots \ldots \ldots$
\end{tabular} \\
\hline
\end{tabular}




\section{TABLE 97.-Germany-YIELD of MILK and PRODUC-} TION of DAIRY PRODUCTS.

[Food conditions in Germany, E.H. Starling, 1919.]

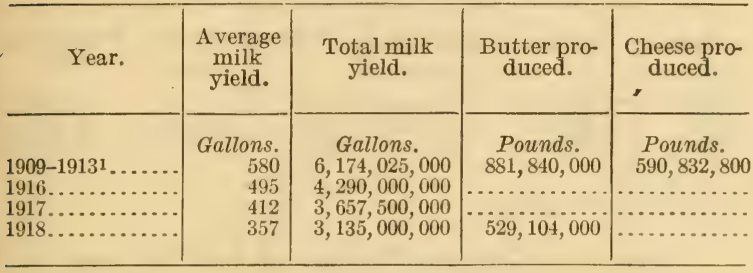

1 A verage.

\section{TABLE 98.-Germany-IMPORTS of DAIRY PROD- UCTS.}

[Auswärtiger Handel Deutschland and Bul. 103, Bureau of Statistics; (YB.).]

\begin{tabular}{|c|c|c|c|c|}
\hline Year. & Butter. & Cheese. & $\begin{array}{l}\text { Condensed } \\
\text { milk. }\end{array}$ & Whole milk. \\
\hline $\begin{array}{l}1897 \ldots \ldots \\
1900 \ldots \\
1910 \ldots \\
1911 \ldots \\
1912 \ldots \\
1913 \ldots \\
1914 \ldots \\
1920^{2} \ldots\end{array}$ & $\begin{array}{r}\text { Pounds. } \\
19,936,946 \\
34,647,494 \\
92,815,865 \\
123,619,418 \\
122,472,143 \\
119,576,189 \\
\hdashline 17,236,965\end{array}$ & $\begin{array}{r}\text { Pounds. } \\
\text { Npt found. } \\
36,689,123 \\
46,011,104 \\
45,954,448 \\
47,276,765 \\
57,902,937 \\
\hdashline 50,343,584\end{array}$ & $\begin{array}{r}\text { Pounds. } \\
3,086 \\
3,748 \\
59,083 \\
52,910 \\
\ldots . .8 \\
3,086 \\
882 \\
9,180,395\end{array}$ & $\begin{array}{r}\text { Pounds. } \\
20,743,700 \\
133,154,098 \\
82,054,110 \\
92,154,264 \\
. . \\
5,602,550 \\
5,515,468\end{array}$ \\
\hline
\end{tabular}

${ }^{1}$ Including cream.

2 Preliminary.

\section{TABLE 99.-Germany-EXPORTS of DAIRY PROD- UCTS.}

[Auswärtiger Handel Deutschland and Bul. 103, Bureau of Statistics; (YB.).]

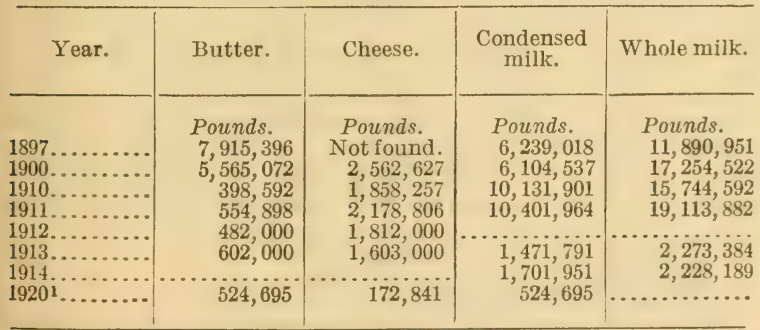

1 Preliminary. 
TABLE 100.-Hungary-Number of COWS and TOTAL
CATTLE.

[Various authorities; (YB.).]

\begin{tabular}{|c|c|c|c|c|c|}
\hline Year. & Cows. & Total cattle. & Year. & Cows. & Totalcattle. \\
\hline $\begin{array}{l}1870 \ldots \ldots \\
1880 \ldots . \\
1895 \ldots\end{array}$ & $\begin{array}{l}2,052,488 \\
2,035,217 \\
2,185,224\end{array}$ & $\begin{array}{l}5,279,193 \\
5,311,378 \\
6,738,365\end{array}$ & $\begin{array}{l}1904 \ldots \\
1909 \ldots \\
1913 \ldots\end{array}$ & $2,500,000$ & $\begin{array}{r}7,319,121 \\
7,152,568 \\
16,045,000\end{array}$ \\
\hline
\end{tabular}

1 And 162,000 buffaloes.

\section{TABLE 101.-India-Number of CATTLE and BUFFALOES.}

[Yearbook, U. S. Department of Agriculture.]

\begin{tabular}{|c|c|c|c|c|c|}
\hline \multirow{2}{*}{ Year. } & \multicolumn{2}{|c|}{ British India. } & \multirow{2}{*}{ Year. } & \multicolumn{2}{|c|}{ Nati e States. } \\
\hline & Cattle. & Buffaloes. & & Cattle: & Buffaloes. \\
\hline $\begin{array}{l}1894-5 \ldots . \\
1899-1900 . \\
1904-5 \ldots \ldots \\
1910-11 \ldots \\
1913-14 \ldots \\
1914-15 \ldots \\
1915-16 \ldots \\
1916-17 \ldots \\
1917-18 \ldots\end{array}$ & $\begin{array}{r}67,045,000 \\
72,666,000 \\
77,111,000 \\
94,664,000 \\
125,042,000 \\
128,310,000 \\
129,742,000 \\
130,087,000 \\
129,876,000\end{array}$ & $\begin{array}{l}11,826,000 \\
12,120,000 \\
12,871,000 \\
16,628,000 \\
18,235,000 \\
19,025,000 \\
19,206,000 \\
19,266,000 \\
19,235,000\end{array}$ & $\begin{array}{l}1900-1 . \\
1904-5 . \\
1909-10 \\
1913-14 \\
1916-17 \\
1917-18 .\end{array}$ & $\begin{array}{r}7,397,000 \\
8,178,000 \\
10,391,000 \\
12,236,000 \\
12,999,000 \\
12,691,000\end{array}$ & $\begin{array}{l}1,228,000 \\
1,347,000 \\
1,559,000 \\
1,765,000 \\
1,802,000 \\
1,863,000\end{array}$ \\
\hline
\end{tabular}

Young buffaloes are included under cattle and not under bufialoes. The heading "Buffaloes" means adult animals.

Figures for the Native States are reported as "not complete."

\section{TABLE 102.-Italy-Number of CATTLE, BUFFALOES, SHEEP, and GOATS.}

[Annuario Statistico Italiano, Census Reports, and L'Italia Agricola; (YB.).]

\begin{tabular}{|c|c|c|c|c|}
\hline Year. & Cattle. & Buffaloes. & Sheep. & Goats. \\
\hline \multirow{4}{*}{ 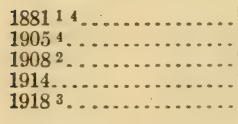 } & \multirow{4}{*}{$\begin{array}{r}4,772,000 \\
5,672,000 \\
6,198,861 \\
6,64 \\
6,239,741\end{array}$} & 11,000 & $8,596,000$ & $2,016,000$ \\
\hline & & 19,366 & ii, 162,926 & $2,714,878$ \\
\hline & & 6,000 & 13,82 & $\begin{array}{l}4,000 \\
3,082,558\end{array}$ \\
\hline & & 24,026 & $11,753,910$ & $3,082,558$ \\
\hline
\end{tabular}

1 Census taken in 1881 was as of Feb. 13.

2 Census taken in 1908 was as of Mar. 10.

3 Census taken in 1918 was as of April.

4 Prior to the census of 1908 the data were taken by sending schedules out to be filled in by the farmers. 


\section{TABLE 103.-Italy-IMPORTS and EXPORTS of DAIRY PRODUCTS.}

[Movimento Commerciale, Italy; (YB.).]

\begin{tabular}{|c|c|c|c|c|c|c|}
\hline \multirow{2}{*}{ Year. } & \multicolumn{2}{|c|}{ Butter. } & \multicolumn{2}{|c|}{ Cheese. } & \multicolumn{2}{|c|}{ Condensed milk. } \\
\hline & Imports. & Exports. & Imports. & Exports. & Imports. & Exports. \\
\hline & Pounds. & Pounds. & Pounds. & Pounds. & Pounds. & Pounds. \\
\hline $\begin{array}{l}1871 . \\
1880\end{array}$ & $\begin{array}{l}287,700 \\
509,273\end{array}$ & $2,213,198$ & $\begin{array}{l}12,553,433 \\
16.512,454\end{array}$ & 3, 887, 151 & . & \\
\hline 1890. & 705,913 & $6,709,921$ & $17,059,195$ & $\begin{array}{r}\text { D, 50, } \\
12,559,386\end{array}$ & & \\
\hline 190 & 429,236 & $14,136,777$ & $9,352,134$ & $26,051,097$ & & \\
\hline $1 \mathrm{~s}$ & 345,020 & $8,295,469$ & $14,760,900$ & $57,515,814$ & 574,960 & $6,615,1$ \\
\hline & $2,516,771$ & $8,147,320$ & $11,915,422$ & $61,402,526$ & 834,882 & $6,629,0$ \\
\hline 1912 & 899,256 & $8,842,650$ & $10,068,629$ & $67,504,856$ & 954,592 & $7,183,6$ \\
\hline 1913 & 909,838 & $6,034,211$ & $12,354,799$ & $72,320,580$ & $1,214,955$ & $5,72 \times, 6$ \\
\hline & 194,005 & $9,310,026$ & $9,837,807$ & $66,003,526$ & $1,111,559$ & $9,998,5$ \\
\hline & 96,561 & $7,488,365$ & $3,471,584$ & $65,761,027$ & $1,335,106$ & $11,575,032$ \\
\hline & 135,803 & 792,113 & 252,206 & $39,322,568$ & 959,001 & $6,906,7$ \\
\hline & 126,324 & 171,518 & 9.259 & $4,336,669$ & $2,064,828$ & 646,3 \\
\hline & 73,193 & 108,687 & $\mathbf{7 4 6 , 0 3 7}$ & 938,278 & $7,210,144$ & 157, \\
\hline & $1,880,087$ & 50,926 & $11,151,308$ & $1,821,441$ & $27,383,341$ & 279,5 \\
\hline & $3,103,856$ & $95,6 \times 0$ & $5,593,337$ & $2,789,921$ & $1,632,727$ & 404,985 \\
\hline
\end{tabular}

\section{TABLE 104.-Japan-Number of COWS, TOTAL CAT- TLE, and MILK COWS.}

[Japanese Yearbook.]

\begin{tabular}{|c|c|c|c|c|c|c|}
\hline Year. & $\begin{array}{l}\text { Native } \\
\text { cows. }\end{array}$ & $\begin{array}{l}\text { Grade } \\
\text { cows. }\end{array}$ & $\begin{array}{c}\text { Foreign } \\
\text { breeds of } \\
\text { cows. }\end{array}$ & $\begin{array}{l}\text { Total } \\
\text { cattle. }\end{array}$ & $\begin{array}{l}\text { Millk } \\
\text { cows. }\end{array}$ & $\begin{array}{c}\text { Average } \\
\text { yield. }\end{array}$ \\
\hline $\begin{array}{l}1899 . . \\
1900 . . \\
1905 . . \\
1910 . . \\
1911 . . \\
1912 . . \\
1913 . \\
1914 . . \\
1915 . \\
1916 . \\
1917 . . \\
1918 . \\
1919 . .\end{array}$ & $\begin{array}{l}685,106 \\
676,600 \\
560,295 \\
569,441 \\
567,973 \\
560,564 \\
565,324 \\
580,907 \\
605,244 \\
590,084 \\
580,770 \\
587,859 \\
612,871\end{array}$ & $\begin{array}{r}70,002 \\
82,715 \\
171,278 \\
322,857 \\
338,278 \\
340,193 \\
331,919 \\
321,137 \\
307,969 \\
301,170 \\
295,626 \\
298,086 \\
304,739\end{array}$ & $\begin{array}{l}14,055 \\
15,343 \\
15,943 \\
18,404 \\
21,345 \\
20,870 \\
20,815 \\
14,625 \\
12,739 \\
12,191 \\
12,690 \\
14,918 \\
15,538\end{array}$ & $\begin{array}{l}1,252,865 \\
1,261,214 \\
1,167,610 \\
1,384,183 \\
1,405,026 \\
1,399,498 \\
1,388,708 \\
1,387,233 \\
1,387,922 \\
1,342,990 \\
1,304,331 \\
1,307,120 \\
1,344,865\end{array}$ & $\begin{array}{r}41,333 \\
44,060 \\
41,943 \\
42,281 \\
43,374 \\
43,645 \\
44,791 \\
45,739 \\
42,035 \\
40,343\end{array}$ & $\begin{array}{r}\text { Pounds. } \\
\ldots \ldots . . . . . . \\
\ldots . . . . . \\
2,581 \\
2,515 \\
2,732 \\
2,705 \\
2,722 \\
2,861 \\
2,871 \\
3,091 \\
3,339 \\
3,303\end{array}$ \\
\hline
\end{tabular}


TABLE 105.-Japan-Quantity of DAIRY PRODUCTS made in factories.

[Japanese Yearbook.]

\begin{tabular}{|c|c|c|c|}
\hline Year. & $\begin{array}{l}\text { Condensed } \\
\text { milk. }\end{array}$ & Butter. & $\begin{array}{l}\text { Artificial } \\
\text { butter. }{ }^{1}\end{array}$ \\
\hline $\begin{array}{l}1915 \ldots \ldots \\
1916 \ldots \ldots \\
1917 \ldots \ldots \ldots \\
1918 \ldots \ldots\end{array}$ & $\begin{array}{r}\text { Pounds. } \\
4,863,170 \\
8,520,541 \\
8,271,925 \\
14,405,419 \\
18,852,588\end{array}$ & $\begin{array}{r}\text { Pounds. } \\
682,282 \\
897,084 \\
1,428,258 \\
1,302,749 \\
1,203,253\end{array}$ & $\begin{array}{r}\text { Pounds. } \\
410,793 \\
481,869 \\
811,687 \\
750,592 \\
907,704\end{array}$ \\
\hline
\end{tabular}

1 Principally vegetable.

\section{TABLE 106.-Netherlands-Number of DAIRY COWS and TOTAL CATTLE.}

[Jaarcijfers Kroninkrijk der Nederlanden, Rijk in Europe.]

\begin{tabular}{|c|c|c|c|c|c|}
\hline Year. & $\begin{array}{l}\text { Dairy } \\
\text { cows. }\end{array}$ & $\begin{array}{c}\text { Total } \\
\text { cattle(YB.) }\end{array}$ & Year. & $\begin{array}{l}\text { Dairy } \\
\text { cows. }\end{array}$ & $\begin{array}{c}\text { Total } \\
\text { attle(YB.) }\end{array}$ \\
\hline $\begin{array}{l}18711 \ldots \ldots \\
18811^{1} \ldots \ldots \\
1891 \ldots \ldots \\
1900 \ldots \ldots\end{array}$ & $\begin{array}{l}911,200 \\
893,900 \\
897,400 \\
965,800\end{array}$ & $\begin{array}{l}1,243,700 \\
1,335,300 \\
1,435,700 \\
1,434,400 \\
1,532,200 \\
1,655,600\end{array}$ & $\begin{array}{l}1910 \ldots . \\
1913 \ldots \\
1917^{2} \ldots \\
1918^{3} \ldots \\
1919^{4} \ldots \\
1920 \ldots\end{array}$ & $\begin{array}{l}1,068,361 \\
1,109,679 \\
1,234,375 \\
1,130,908 \\
1,232,264\end{array}$ & $\begin{array}{r}2,026,913 \\
2,096,599 \\
2,301,532 \\
2,043,872 \\
1,968,609 \\
\ldots . \ldots . \ldots .\end{array}$ \\
\hline
\end{tabular}

I The number of dairy cows shown for 1871 and 1481 are the averages for the 10-year periods beginning on those dates.

3 Number in April. 3 Number in August. 4 Number in March.

\section{TABLE 107.-Netherlands-PRODUCTION and CON- SUMPTION OF FACTORY BUTTER.}

[Commerce Reports, No. 245, Oct. 18, 1920.]

\begin{tabular}{|c|c|c|c|c|}
\hline \multirow[b]{2}{*}{ Month. } & \multicolumn{2}{|c|}{1916.} & \multicolumn{2}{|c|}{1917.} \\
\hline & Production. & $\begin{array}{c}\text { Consump- } \\
\text { tion. }\end{array}$ & Production. & $\begin{array}{l}\text { Consump- } \\
\text { tion. }\end{array}$ \\
\hline 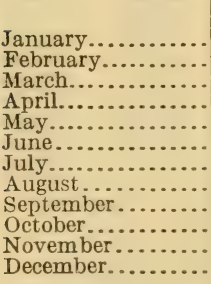 & $\begin{array}{r}\text { Pounds. } \\
7,054,720 \\
7,054,720 \\
11,684,3 \varepsilon 0 \\
11,243,460 \\
14,109,440 \\
17,195,8 \varepsilon 0 \\
12,566,220 \\
14,770,820 \\
10,582,080 \\
9,038,860 \\
9,479,780 \\
6,393,340\end{array}$ & $\begin{array}{l}\text { Pounds. } \\
3,968,280 \\
4,188,740 \\
4,409,200 \\
3,747,820 \\
4,409,200 \\
6,613,800 \\
6,393,340 \\
8,157,020 \\
6,613,800 \\
6,172,880 \\
5,952,420 \\
4,409,200\end{array}$ & $\begin{array}{r}\text { Pounds. } \\
5,731,960 \\
5,731,960 \\
9,700,240 \\
9,259,320 \\
11,243,460 \\
15,432,200 \\
11,023,000 \\
13,227,600 \\
9,920,700 \\
8,597,940 \\
7,716,100 \\
5,070,580\end{array}$ & $\begin{array}{r}\text { Pounds. } \\
4,629,660 \\
5,070,580 \\
4,409,200 \\
3,747,820 \\
4,188,740 \\
7,054,720 \\
6,393,340 \\
10,141,160 \\
6,834,260 \\
6,393,340 \\
3,086,440 \\
1,984,140\end{array}$ \\
\hline Total....... & $131,173,700$ & $65,035,700$ & $112,655,060$ & $63,933,400$ \\
\hline
\end{tabular}




\section{TABLE 107.-Netherlands-PRODUCTION and CON- SUMPTION of FACTORY BUTTER-Continued.}

\begin{tabular}{|c|c|c|c|c|}
\hline \multirow{2}{*}{ Month. } & \multicolumn{2}{|c|}{1918.} & \multicolumn{2}{|c|}{1919.} \\
\hline & Production. & $\begin{array}{l}\text { Consump- } \\
\text { tion. }\end{array}$ & Production. & $\begin{array}{l}\text { Consump- } \\
\text { tion. }\end{array}$ \\
\hline 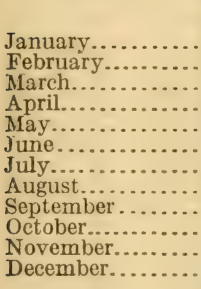 & $\begin{array}{r}\text { Pounds. } \\
3,968,200 \\
3,086,440 \\
4,629,660 \\
5,511,500 \\
9,479,780 \\
13,007,140 \\
9,259,320 \\
11,243,460 \\
8,377,480 \\
7,054,720 \\
7,275,180 \\
5,070,580\end{array}$ & $\begin{array}{l}\text { Pounds. } \\
2,425,060 \\
2,425,060 \\
3,527,360 \\
4,629,660 \\
5,511,500 \\
8,597,940 \\
5,731,960 \\
6,172,880 \\
4,850,120 \\
5,070,580 \\
5,291,040 \\
3,968,280\end{array}$ & $\begin{array}{r}\text { Pounds. } \\
3,747,820 \\
3,086,440 \\
4,629,660 \\
5,731,960 \\
8,818,400 \\
14,109,440 \\
10,582,080 \\
12,786,680 \\
9,038,860 \\
8,377,480 \\
8,818,400 \\
6,172,880\end{array}$ & $\begin{array}{l}\text { Pounds. } \\
3,086,440 \\
3,527,360 \\
4,409,200 \\
3,306,900 \\
5,291,040 \\
7,716,100 \\
7,936,560 \\
8,377,480 \\
6,393,340 \\
6,613,800 \\
5,291,040 \\
2,865,980\end{array}$ \\
\hline Total..... & $87,963,540$ & $58,201,440$ & $95,900,100$ & $64,815,240$ \\
\hline
\end{tabular}

1920.

Month.

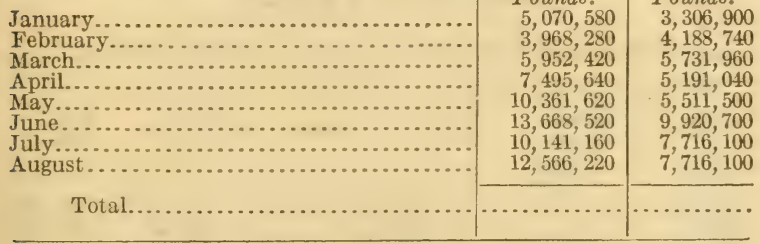

Extract from consular report: "Despite constantly increasing production, consumption thus far during the current year (1920) has amounted to 71 per cent of the output; in the full year 1919, it was 67.6 per cent; in 1918, 66.1 per cent; in 1917, 56.8 per cent; and in 1916, only 49.6 per cent. Production in 1920 has been at the rate of $103,836,660$ pounds, and consumption at the rate of $7+1,294,560$ pounds for the entire year." 
TABLE 108.-Netherlands-IMPORTS and EXPORTS of BUTTER and CHEESE.

[Statistick van den in-uit-en doorvoer; (YB.).]

\begin{tabular}{|c|c|c|c|c|}
\hline \multirow{2}{*}{ Year. } & \multicolumn{2}{|c|}{ Butter. } & \multicolumn{2}{|c|}{ Cheese. } \\
\hline & Imports. & Exports. & Imports. & Exports. \\
\hline 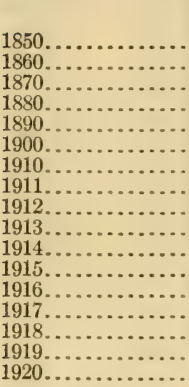 & $\begin{array}{r}\text { Pounds. } \\
231,890 \\
1,572,043 \\
6,861,284 \\
2,759,718 \\
8,834,868 \\
1,634,102 \\
4,491,879 \\
6,038,399 \\
4,636,273 \\
5,529,137 \\
3,880,444 \\
904,693 \\
992,070 \\
52,339 \\
42,782 \\
614,753 \\
130,675\end{array}$ & $\begin{array}{r}\text { Pounds. } \\
26,491,049 \\
39,331,100 \\
147,865,455 \\
179,479,604 \\
87,203,279 \\
49,762,641 \\
72,456,276 \\
66,512,782 \\
86,307,885 \\
81,702,476 \\
81,406,790 \\
93,351,626 \\
78,997,431 \\
54,214,930 \\
5,415,259 \\
30,242,048 \\
45,575,696\end{array}$ & $\begin{array}{r}\text { Pounds. } \\
287,023 \\
611,563 \\
512,230 \\
635,187 \\
655,001 \\
578,990 \\
676,724 \\
649,709 \\
652,996 \\
663,397 \\
2,027,819 \\
339,559 \\
\ldots 10,163 \\
730 \\
42,370 \\
488,705\end{array}$ & $\begin{array}{r}\text { Pounds. } \\
49,184,300 \\
61,021,134 \\
64,445,875 \\
61,857,233 \\
66,832,431 \\
101,208,865 \\
122,771,456 \\
113,607,416 \\
131,107,075 \\
145,336,751 \\
149,573,576 \\
190,333,870 \\
199,108,449 \\
123,633,961 \\
32,893,251 \\
27,371,522 \\
99,737,645\end{array}$ \\
\hline
\end{tabular}

1 Oleomargarin and butter reported together from 1870 to 1889.

\section{TABLE 109.-New Zealand-Number of DAIRY COWS and TOTAL CATTLE.}

[New Zealand Yearbook and Statistical Abstract; (YB.).]

\begin{tabular}{|c|c|c|c|c|c|}
\hline Year. & $\begin{array}{l}\text { Dairy } \\
\text { cows. }\end{array}$ & $\begin{array}{l}\text { Total } \\
\text { cattle. }\end{array}$ & Year. & $\begin{array}{l}\text { Dairy } \\
\text { cows. }\end{array}$ & $\begin{array}{l}\text { Total } \\
\text { cattle. }\end{array}$ \\
\hline $\begin{array}{l}1861 \ldots \ldots \\
1864 \ldots \ldots \\
1871 \ldots \ldots \\
1874 \ldots . \\
1881 \ldots . \\
1885 \ldots \\
1890 \ldots . \\
1895 \ldots . \\
1900 \ldots .\end{array}$ & 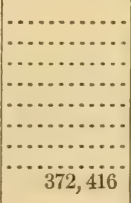 & $\begin{array}{r}193,285 \\
249,760 \\
436,592 \\
494,917 \\
698,637 \\
853,358 \\
831,831 \\
1,047,901 \\
1,256,680\end{array}$ & $\begin{array}{l}1905 \ldots . \\
1910 \ldots \ldots \\
1911 \ldots \ldots \\
19161 \ldots \\
19171 \ldots \\
1918 \ldots \ldots \\
1919 \ldots \ldots \\
1920 \ldots \ldots \\
1921 \ldots \ldots\end{array}$ & $\begin{array}{l}517,720 \\
633,733 \\
536,629 \\
732,931 \\
777,439 \\
793,215 \\
820,451 \\
882,854 \\
993,472\end{array}$ & \begin{tabular}{l}
$1,810,936$ \\
$2,020,171$ \\
\hdashline $2,417,191$ \\
$2,575,230$ \\
$2,869,465$ \\
$3,035,478$ \\
$3,059,445$ \\
$3,112,742$
\end{tabular} \\
\hline
\end{tabular}

1 Number on June 20. 


\section{TABLE 110.-New Zealand-EXPORTS of DAIRY PRODUCTS.}

[Trade and Navigation Reports, Abstract of Statistics of New Zealand, and 1915 Yearbook of New Zealand; (YB.).]

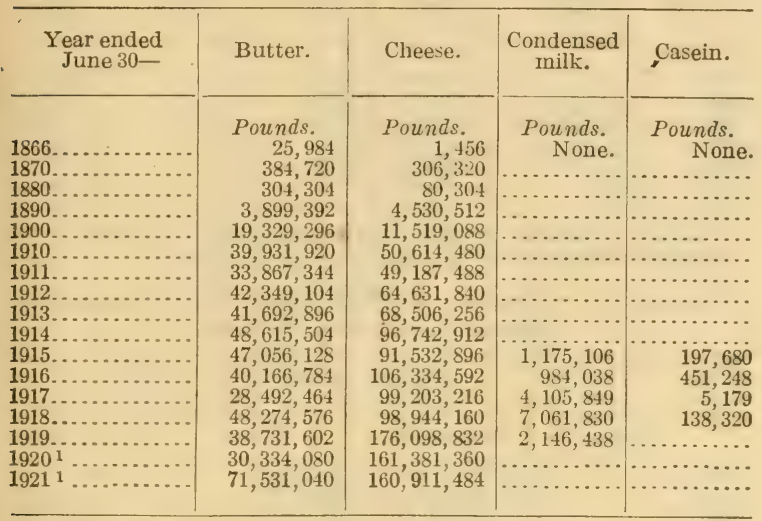

1 Year ended July 31.

\section{TABLE 111.-Norway-Number of COWS, TOTAL CAT- TLE, and REINDEER.}

[Norges Jordbrug, and Statistisk Aarbok; (YB.).]

\begin{tabular}{|c|c|c|c|}
\hline Year. & Cows. & Total cattle. & Reindeer. \\
\hline $\begin{array}{l}1815 \\
1855 \\
1865 \\
1875 \\
1890 \\
1900 \\
1907{ }^{2} \\
190 \\
1910 \\
1914 \\
1915 \\
1916 \\
1916 \\
1918\end{array}$ & $\begin{array}{r}741,598 \\
706,925 \\
689,563 \\
727,898 \\
755,483 \\
782,000\end{array}$ & $\begin{array}{r}842,568 \\
949,935 \\
953,036 \\
1,016,617 \\
1,006,499 \\
950,201 \\
1,039,000 \\
1,134,613 \\
1,146,000 \\
1,121,000 \\
1,126,000 \\
1,085,707 \\
1,053,743\end{array}$ & $\begin{array}{r}90,273 \\
116,891 \\
101,768 \\
96,567 \\
170,134 \\
109,000 \\
142,623 \\
(2) \\
(2) \\
(2) \\
(2) \\
(2)\end{array}$ \\
\hline
\end{tabular}

${ }^{1}$ Beginning with 1907 figures are for Sept. 30; prior to that, December.

${ }^{2}$ Not officially reported after 1907. 


\section{TABLE 112.-Roumania-Number of DAIRY COWS and} TOTAL CATTLE.

[Estatistical Agricola a Romaniei; (YB.).]

\begin{tabular}{|c|c|c|c|c|c|}
\hline Year. & $\begin{array}{l}\text { Dairy } \\
\text { cows. }\end{array}$ & $\begin{array}{l}\text { Total } \\
\text { cattle. }\end{array}$ & Year. & $\begin{array}{l}\text { Dairy } \\
\text { cows. }\end{array}$ & $\begin{array}{l}\text { Total } \\
\text { eattle. }\end{array}$ \\
\hline $\begin{array}{l}1884 \ldots \ldots \\
1888 \ldots . . \\
1890 \ldots . \\
1896 \ldots . \\
1900 \ldots . \\
1907 \ldots . .\end{array}$ & $\begin{array}{r}820,510 \\
\ldots 01,227 \\
\ldots \ldots \ldots \ldots\end{array}$ & $\begin{array}{r}2,376,056 \\
2,406,071 \\
2,520,380 \\
2,138,315 \\
12,558,526 \\
12,585,205\end{array}$ & $\begin{array}{l}1911 \ldots . . . \\
1916 . \ldots . \\
1917 \ldots \ldots \\
1918 \ldots \ldots \\
1919 \ldots \ldots \\
1920 \ldots \ldots\end{array}$ & 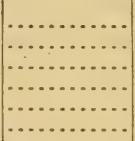 & $\begin{array}{r}12,666,945 \\
1 \\
2,938,000 \\
1,050,000 \\
\ldots \\
1,125,000 \\
\ldots \ldots \ldots . .\end{array}$ \\
\hline
\end{tabular}

${ }^{1}$ Including buffaloes. In 1900 there were 25,750 cow buffaloes, 10,482 young buffaloes, and 7,243 other buffaloes in Roumania.

\section{TABLE 113.-Russia-TOTAL CATTLE in European Russia (including Poland).}

[Report 109, U. S. Dept. of Agric., and Statistical Abstract of Great Britain; (YB.].

\begin{tabular}{|c|c|c|c|}
\hline . Year. & Total cattle. & Year. & Total cattle. \\
\hline 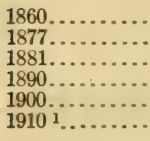 & $\begin{array}{l}21,408,800 \\
27,322,219 \\
27,177,000 \\
28,541,400 \\
34,483,900 \\
36,301,602\end{array}$ & 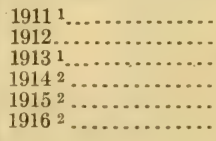 & $\begin{array}{l}36,237,688 \\
34,571,750 \\
37,165,255 \\
32,704,000 \\
32,886,000 \\
38,373,000\end{array}$ \\
\hline
\end{tabular}

1 Includes northern Caucasia.

2 U. S. Dept. of Agric., Bulletin 987.

There are no available figures on the number of cows or dairy cows in Russia.

Siberia in 1914 had $17,334,000$ cattle.

The number of reindeer in Russia in 1913 was $605,000$.

\section{TABLE 114.-Russia (including Siberia)-Production of DAIRY PRODUCTS.}

[Reports of railroads; Enquête sur l'Industrie, Paris.]

\begin{tabular}{|c|c|c|c|}
\hline Year. & Butter. & $\begin{array}{l}\text { Milk and } \\
\text { cream. }\end{array}$ & $\begin{array}{l}\text { Other dairy } \\
\text { produets. }\end{array}$ \\
\hline 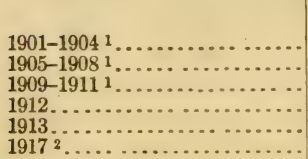 & $\begin{array}{c}\text { Pounds. } \\
190,810,300 \\
226,844,230 \\
275,286,240 \\
306,414,650 \\
326,253,150 \\
162,507,600\end{array}$ & $\begin{array}{c}\text { Pounds. } \\
122,421,580 \\
154,920,065 \\
238,639,120 \\
442,939,600 \\
525,972,740\end{array}$ & $\begin{array}{r}\text { Pounds. } \\
60,128,690 \\
71,923,580 \\
97,713,630 \\
113,728,710 \\
124,549,710\end{array}$ \\
\hline
\end{tabular}

1 Yearly average.

2 From Russian Chamber of Commerce at Paris (Int. Rev. of the Sci. and Prac. of Agric., Rome, July, August, September, 1919, p. 1000). 


\section{TABLE 115.-Russia (including Siberia)-EXPORTS of BUTTER and CHEESE.}

[Russian Commerce Reports; (YB.).]

\begin{tabular}{|c|c|c|c|c|c|}
\hline Year. & $\begin{array}{l}\text { Butter } \\
\text { exports. }\end{array}$ & $\begin{array}{l}\text { Cheese } \\
\text { exports. }\end{array}$ & Year. & $\begin{array}{c}\text { Butter } \\
\text { exports. }\end{array}$ & $\begin{array}{l}\text { Cheese } \\
\text { exports. } 1\end{array}$ \\
\hline $\begin{array}{l}1860-1870^{2} \\
1871-18752 \\
1876-1880 \\
1885-1888 \\
1894-1898^{2} \\
1900 \ldots \ldots \\
1910 \ldots \ldots \\
1911 \ldots \ldots \\
1912 \ldots \ldots\end{array}$ & $\begin{array}{r}\text { Pounds. } \\
6,842,479 \\
5,875,803 \\
6,694,592 \\
11,650,610 \\
14,896,910 \\
42,923,300 \\
124,365,658 \\
168,704,448 \\
160,770,972\end{array}$ & $\begin{array}{r}\text { Pounds. } \\
216,420 \\
36,070 \\
1,089,314 \\
1,861,212 \\
1,410,337 \\
1,490,047 \\
1,423,675 \\
2,102,162 \\
2,088,764\end{array}$ & $\begin{array}{l}1913 \ldots \\
1914 \ldots \\
1915 \ldots \\
1916 \ldots \\
1917 \ldots \\
1918 \ldots \\
1919 \ldots \\
1920 \ldots\end{array}$ & $\begin{array}{r}\text { Pounds. } \\
172,003,064 \\
118,996,623 \\
119,358,799 \\
321,668 \\
21,668 \\
\ldots . . . . . . . .\end{array}$ & 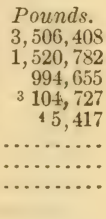 \\
\hline
\end{tabular}

1 Not including "curd."

2 Average annual export.

3 Preliminary figures.

4 European frontier only. Figures from U. S. War Trade Board.

\section{TABLE 116.-South Africa (British)-Production of BUTTER and CHEESE.}

[Reports of Secretary of Agriculture, South Africa.]

\begin{tabular}{|c|c|c|c|c|c|}
\hline Year. & Butter. & Cheese. & Year. & Butter. & Cheese. \\
\hline $\begin{array}{l}1913 . . \\
1914 . . \\
1915 . . \\
1916 \ldots\end{array}$ & $\begin{array}{l}\text { Pounds. } \\
10,741,000 \\
10,682,000 \\
13,500,000\end{array}$ & $\begin{array}{r}\text { Pounds. } \\
520,849 \\
605,686 \\
1,098,184\end{array}$ & $\begin{array}{l}1917 . . . \\
1918 . . . \\
1919 . .\end{array}$ & $\begin{array}{l}\text { Pounds. } \\
16,013,557 \\
117,935,680 \\
121,748,160\end{array}$ & 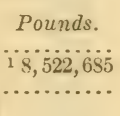 \\
\hline
\end{tabular}

1 Preliminary figures.

TABLE 117.-Spain-Number of CATTLE and GOATS.

[Various sources.]

\begin{tabular}{|c|c|c|c|c|c|}
\hline Year. & $\begin{array}{c}\text { Total } \\
\text { cattle(YB). }\end{array}$ & $\begin{array}{l}\text { Total } \\
\text { goats. }\end{array}$ & Year. & $\begin{array}{c}\text { Total } \\
\text { cattle(YB). }\end{array}$ & $\begin{array}{l}\text { Total } \\
\text { goats. }\end{array}$ \\
\hline $\begin{array}{l}1865 \ldots . . \\
1891 \ldots \\
1905 \ldots \\
1906 \ldots \\
1910 \ldots \\
1911 \ldots \\
1912 \ldots \\
1913 \ldots \\
\end{array}$ & $\begin{array}{l}2,904,598 \\
2,217,659 \\
2,075,142 \\
2,497,000 \\
2,368,767 \\
2,541,112 \\
2,561,891 \\
2,879,000\end{array}$ & \begin{tabular}{r}
$2,534,000$ \\
\hdashline $2,440,000$ \\
$3,216,489$ \\
$3,369,624$ \\
$3,116,226$ \\
$3,394,000$
\end{tabular} & 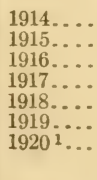 & $\begin{array}{l}2,743,000 \\
2,926,170 \\
3,070,903 \\
3,233,000 \\
3,712,000 \\
3,173,577 \\
3,396,573\end{array}$ & $\begin{array}{l}3,265,000 \\
3,216,682 \\
3,207,360 \\
4,1 \times 2,000 \\
4,476,000 \\
3,685,808 \\
3,790,656\end{array}$ \\
\hline
\end{tabular}

1 Milk cows, 228,659 . 


\section{TABLE 118.-Sweden-Number of COWS and TOTAL CATTLE.}

[Statistik Arsbok and Statistik Tidskrift.]

\begin{tabular}{|c|c|c|c|c|c|}
\hline Year. & Cows. & $\begin{array}{c}\text { Total } \\
\text { cattle(YB). }\end{array}$ & Year. & Cows. & $\begin{array}{c}\text { Total } \\
\text { cattle(YB). }\end{array}$ \\
\hline $\begin{array}{l}1865 \ldots \ldots \\
1870 \ldots \ldots \\
1880 \ldots \ldots \\
1890 \ldots \ldots \\
1900 \ldots \ldots \\
1910 \ldots \ldots \\
1911 \ldots \ldots\end{array}$ & $\begin{array}{l}1,185,556 \\
1,231,477 \\
1,409,236 \\
1,578,927 \\
1,764,819 \\
1,861,219 \\
1,837,035\end{array}$ & $\begin{array}{l}1,924,354 \\
1,965,900 \\
2,227,757 \\
2,399,491 \\
2,582,555 \\
2,747,526 \\
2,689,609\end{array}$ & $\begin{array}{l}1913 \ldots \ldots \\
1914 \ldots . \\
1915 \ldots \\
19161 \ldots \\
19171 \ldots \\
19181 \ldots \\
19191 \ldots\end{array}$ & $\begin{array}{l}1,841,991 \\
1,815,989 \\
1,841,508 \\
1,769,689 \\
1,775,353 \\
1,634,982 \\
1,607,060\end{array}$ & $\begin{array}{l}2,720,741 \\
2,761,000 \\
2,883,884 \\
2,913,159 \\
3,020,381 \\
2,584,159 \\
2,550,828\end{array}$ \\
\hline
\end{tabular}

1 June 1.

The number of reindeer in Sweden in 1918 was 275,084 .

TABLE 119.-Sweden-DAIRY PRODUCTS in factories. [Statistik Arsbok.]

\begin{tabular}{|c|c|c|c|c|}
\hline Year. & $\begin{array}{l}\text { Milk delivered } \\
\text { to dairies. }\end{array}$ & $\begin{array}{l}\text { Number } \\
\text { of butter } \\
\text { factories. }\end{array}$ & Butter made. & $\begin{array}{l}\text { Cheese } \\
\text { made. }\end{array}$ \\
\hline $\begin{array}{l}1890 \ldots \ldots \\
1895 \ldots \ldots \\
1900 \ldots \ldots \\
1905 \ldots \ldots \\
1910 \ldots \ldots \\
1913 \ldots \ldots \\
1914 \ldots \ldots \\
1915 \ldots \ldots \\
1916 \ldots \ldots \ldots \\
1917 \ldots \ldots \ldots \\
1918 \ldots \ldots \\
1919 \ldots \ldots \\
\end{array}$ & $\begin{array}{c}\text { Pounds. } \\
1,115,774,074 \\
1,634,582,991 \\
1,856,891,758 \\
2,023,987,671 \\
2,533,570,352 \\
2,879,401,605 \\
2,766,911,890 \\
2,376,434,915 \\
2,509,339,653 \\
2,085,194,455 \\
1,289,521,246 \\
1,560,534,928\end{array}$ & $\begin{array}{l}1,562 \\
1,793 \\
1,688 \\
1,575 \\
1,416 \\
1,650 \\
1,627 \\
1,663 \\
1,698 \\
1,762 \\
1,495 \\
1,450\end{array}$ & $\begin{array}{l}\text { Pounds. } \\
35,479,510 \\
54,953,356 \\
57,570,964 \\
62,727,497 \\
72,616,094 \\
70,339,968 \\
72,615,115 \\
60,597,840 \\
62,264,518 \\
45,551,450 \\
23,454,739 \\
27,409,792\end{array}$ & $\begin{array}{l}\text { Pounds. } \\
14,538,412 \\
15,243,652 \\
16,392,215 \\
19,043,775 \\
22,345,956 \\
32,138,659 \\
28,869,237 \\
24,127,142 \\
28,505,478 \\
22,630,219 \\
11,798,815 \\
17,603,731\end{array}$ \\
\hline
\end{tabular}

The term "dairies" is used throughout Europe for factories which receive milk and cream for any purpose; but generally it refers to butter and cheese factories. 


\section{TABLE 120.-Sweden-IMPORTS and EXPORTS of BUTTER and CHEESE.}

[Swedish Commerce Reports, Bidrag till Sveriges Officiala; (YB.).]

\begin{tabular}{|c|c|c|c|c|}
\hline \multirow{2}{*}{ Year. } & \multicolumn{2}{|c|}{ Butter. } & \multicolumn{2}{|c|}{ Cheese. } \\
\hline & Imports. & Exports. & Imports. & Exports. \\
\hline 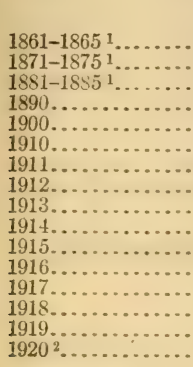 & $\begin{array}{r}\text { Pounds. } \\
2,352,000 \\
3,348,000 \\
5,992,000 \\
3,920,000 \\
1,096,678 \\
205,352 \\
343,029 \\
273,000 \\
432,071 \\
185,879 \\
29,848 \\
61,059 \\
15,756,261 \\
11,425,615 \\
13,846,436 \\
16,940,874\end{array}$ & $\begin{array}{r}\text { Pounds. } \\
369,600 \\
6,955,000 \\
17,654,000 \\
33,163,200 \\
42,246,271 \\
47,949,356 \\
48,888,521 \\
46,818,477 \\
43,329,909 \\
41,941,296 \\
41,532,093 \\
28,703,513 \\
3,117 \\
3,302 \\
76,233 \\
53,417\end{array}$ & \begin{tabular}{r} 
Pounds. \\
$\ldots . . . . . . .$. \\
\hdashline$\ldots . . . . . .$. \\
\hdashline 430,080 \\
710,593 \\
707,677 \\
742,950 \\
$1,258,827$ \\
$1,213,758$ \\
764,864 \\
$1,148,233$ \\
285,855 \\
$1,208,363$ \\
423,715 \\
$3,575,885$ \\
$5,511,985$
\end{tabular} & $\begin{array}{r}\text { Pounds. } \\
\ldots . . . . . . . . \\
\ldots . . . . . . . \\
316,969 \\
9,163 \\
51,583 \\
6,393 \\
11,971 \\
6,045 \\
69,196 \\
627,978 \\
992 \\
699 \\
172 \\
12,281 \\
\ldots . . . . . .\end{array}$ \\
\hline
\end{tabular}

1 Average.

Preliminary figures.

\section{TABLE 121.-Switzerland-Number of DAIRY COWS, TOTAL CATTLE, and GOATS.}

[Statistisches Jahrbuch der Schweiz; also Recensement Fédéral du Bétail; (YB.).]

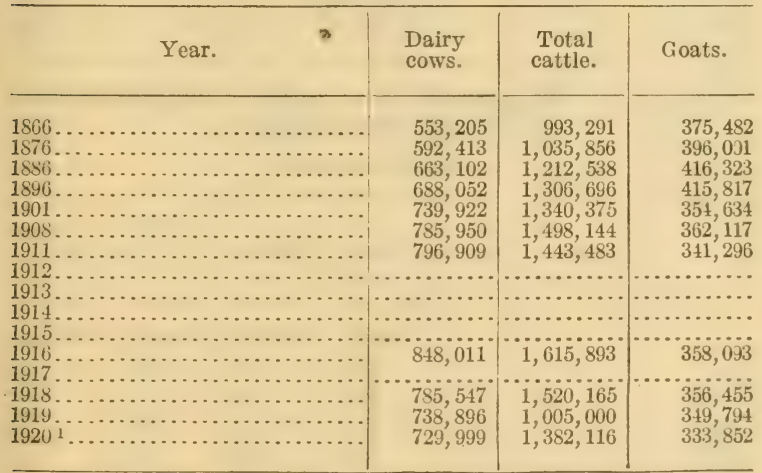

1. On April 21. 
TABLE 122.-Switzerland-PRODUCTION of MILK as estimated officially.

[Swiss Yearbook.]

\begin{tabular}{|c|c|c|c|}
\hline Year. & Cows' milk. & Goats' milk. & Total milk. \\
\hline $\begin{array}{l}1911 \ldots \ldots \ldots \\
1912 \ldots \ldots \\
1913 \ldots \ldots \\
1914 \ldots \ldots \\
1915 \ldots \ldots \\
1916 \ldots \ldots \\
1917 \ldots \ldots \\
1918 \ldots \ldots \ldots\end{array}$ & $\begin{array}{c}\text { Pounds. } \\
5,224,902,000 \\
5,533,546,000 \\
5,798,098,000 \\
5,886,282,000 \\
5,533,546,000 \\
5,423,316,000 \\
4,585,568,000 \\
3,968,280,000\end{array}$ & $\begin{array}{c}\text { Pounds. } \\
209,437,000 \\
209,437,000 \\
209,437,000 \\
220,460,000 \\
220,460,000 \\
220,460,000 \\
220,460,000 \\
220,460,000\end{array}$ & $\begin{array}{c}\text { Pounds. } \\
5,445,362,000 \\
5,754,006,000 \\
6,018,558,000 \\
6,106,742,000 \\
5,731,960,000 \\
5,643,776,000 \\
4,806,028,000 \\
4,188,740,000\end{array}$ \\
\hline
\end{tabular}

These estimates were made in terms of million quintals, to the nearest tenth; hence quantities are not exact and can be considered only as generally representative.

\section{TABLE 123.-Switzerland-DAIRY PRODUCTS manufactured.}

[Swiss Yearbook.]

\begin{tabular}{|c|c|c|c|}
\hline Year. & Cheese. & Butter. & $\begin{array}{l}\text { Condensed } \\
\text { milk. }\end{array}$ \\
\hline 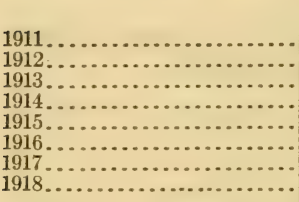 & $\begin{array}{r}\text { Pounds. } \\
144,401,300 \\
170,195,120 \\
180,556,740 \\
174,824,780 \\
127,425,880 \\
110,891,380 \\
71,429,040 \\
63,051,560\end{array}$ & $\begin{array}{l}\text { Pounds. } \\
31,525,780 \\
35,494,060 \\
37,257,740 \\
33,478,200 \\
36,596,360 \\
37,037,280 \\
30,864,400 \\
28,880,260\end{array}$ & $\begin{array}{r}\text { Pounds. } \\
99,207,000 \\
99,207,000 \\
88,144,000 \\
121,253,000 \\
110,230,000 \\
99,207,000 \\
66,138,000 \\
55,115,000\end{array}$ \\
\hline
\end{tabular}

TABLE 124.-Switzerland-Per capita CONSUMPTION of DAIRY PRODUCTS.

[Swiss Yearbook.]

\begin{tabular}{|c|c|c|c|c|}
\hline Year. & Cheese. & Butter. & $\begin{array}{l}\text { Con- } \\
\text { densed } \\
\text { milk. }\end{array}$ & $\begin{array}{l}\text { Whole } \\
\text { milk. }\end{array}$ \\
\hline 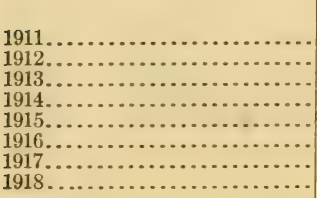 & $\begin{array}{r}\text { Pounds. } \\
22.0 \\
28.9 \\
28.2 \\
26.4 \\
15.0 \\
17.4 \\
14.8 \\
16.1\end{array}$ & $\begin{array}{r}\text { Pounds. } \\
11.5 \\
11.2 \\
12.6 \\
12.1 \\
11.0 \\
9.9 \\
8.2 \\
7.5\end{array}$ & $\begin{array}{r}\text { Pounds. } \\
13.2 \\
8.8 \\
22.0 \\
15.4 \\
13.2 \\
11.0 \\
\ldots . . .\end{array}$ & $\begin{array}{r}\text { Gallons. } \\
64.3 \\
64.3 \\
67.2 \\
67.4 \\
67.7 \\
71.8 \\
67.5 \\
51.3\end{array}$ \\
\hline
\end{tabular}




\section{5}

\section{TABLE 125.-Switzerland-IMPORTS of DAIRY PRODUCTS.}

[Swiss Commerce Repòrts, (YB.).]

\begin{tabular}{|c|c|c|c|c|}
\hline Year. & Fresh milk. & Butter. & Cheese. & $\begin{array}{l}\text { Condensed } \\
\text { milk. }\end{array}$ \\
\hline $\begin{array}{l}1885 \ldots \ldots \ldots \\
1890 \ldots \ldots \\
1900 \ldots \ldots \\
1910 \ldots \ldots \\
1911 \ldots \ldots \\
1912 \ldots \ldots \\
1913 \ldots \ldots \\
1914 \ldots \ldots \\
1915 \ldots \ldots \\
1916 \ldots \ldots \\
1917 \ldots \ldots \ldots \\
1918 \ldots \ldots \\
1919 \ldots \ldots \\
1920 \ldots \ldots \\
\end{array}$ & $\begin{array}{r}\text { Pounds. } \\
246,033 \\
12,996,117 \\
16,037,865 \\
20,110,802 \\
23,858,181 \\
25,994,659 \\
27,649,211 \\
19,270,850 \\
15,510,463 \\
9,773,653 \\
7,655,474 \\
7,199,201 \\
6,225,570 \\
14,149,784\end{array}$ & $\begin{array}{r}\text { Pounds. } \\
2,621,931 \\
3,556,681 \\
4,249,146 \\
11,062,683 \\
12,097,742 \\
11,930,925 \\
11,155,906 \\
8,900,411 \\
5,699,773 \\
946,214 \\
369,491 \\
51,454 \\
13,250,307 \\
18,253,647\end{array}$ & $\begin{array}{r}\text { Pounds. } \\
2,854,957 \\
3,182,561 \\
5,171,991 \\
6,308,683 \\
7,643,789 \\
7,995,202 \\
7,763,058 \\
4,717,403 \\
3,409,854 \\
427,251 \\
213,626 \\
86,640 \\
995,817 \\
4,368,000\end{array}$ & 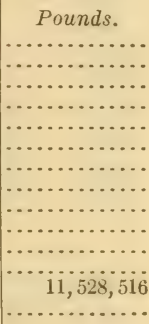 \\
\hline
\end{tabular}

\section{TABLE 126.- Switzerland-EXPORTS of DAIRY PRODUCTS.}

[Swiss Commerce Reports, (YB.).]

\begin{tabular}{|c|c|c|c|c|}
\hline Year. & Fresh milk. & Butter. & Cheese. & $\begin{array}{l}\text { Condensed } \\
\text { milk. }\end{array}$ \\
\hline $\begin{array}{l}1900 \ldots \ldots \\
1910 \ldots \ldots \\
1911 \ldots \ldots \\
1912 \ldots \ldots \\
1913 \ldots \ldots \\
1914 \ldots \ldots \\
1915 \ldots \ldots \\
1916 \ldots \ldots \\
1917 \ldots \ldots \\
1918 \ldots \ldots \\
1919 \ldots \ldots \\
1920 \ldots \ldots\end{array}$ & $\begin{array}{r}\text { Pounds. } \\
8,959,274 \\
31,063,475 \\
33,598,986 \\
35,603,408 \\
39,942,502 \\
37,642,884 \\
41,199,124 \\
31,912,689 \\
23,062,325 \\
20,739,113 \\
20,562,084\end{array}$ & $\begin{array}{r}\text { Pounds. } \\
217,594 \\
172,400 \\
193,343 \\
203,705 \\
192,652 \\
217,374 \\
148,370 \\
2,616 \\
220 \\
4.140\end{array}$ & $\begin{array}{l}\text { Pounds. } \\
60,265,166 \\
69,391,549 \\
66,593,470 \\
66,435,401 \\
79,649,552 \\
77,572,599 \\
74,774,961 \\
47,214,815 \\
12,861,416 \\
2,680,353 \\
1,369,056\end{array}$ & $\begin{array}{r}\text { Pounds. } \\
62,387,094 \\
63,586,876 \\
85,975,873 \\
91,978,558 \\
83,415,269 \\
100,070,762 \\
96,300,455 \\
76,300,455 \\
187,686,642 \\
43,820,394 \\
22,045,559\end{array}$ \\
\hline
\end{tabular}

1 Figures for condensed milk for 1917 taken from Swiss Yearbook. 
TABLE 127.- United Kingdom of Great Britain and Ireland-Number of COWS and TOTAL CATTLE in the United Kingdom, including the Channel Islands and the Isle of Man.

[British Statistical Abstract, and Board of Agriculture and Fisheries of England; (YB.).]

\begin{tabular}{|c|c|c|c|c|c|}
\hline $\begin{array}{c}\text { Year } \\
\text { (June). }\end{array}$ & Cows. & $\begin{array}{l}\text { Total } \\
\text { cattle. }\end{array}$ & $\begin{array}{c}\text { Year } \\
\text { (June). }\end{array}$ & Cows. & $\begin{array}{l}\text { Total } \\
\text { cattle. }\end{array}$ \\
\hline $\begin{array}{l}1875 \ldots \ldots \\
1880 \ldots \ldots \\
1890 \ldots \ldots \\
1900 \ldots \ldots \\
1910 \ldots . \\
1911 \ldots \ldots \\
1912 \ldots \ldots \\
1913 \ldots \ldots\end{array}$ & $\begin{array}{l}3,783,607 \\
3,639,755 \\
3,956,321 \\
4,096,682 \\
4,342,186 \\
4,407,800 \\
4,400,816 \\
4,317,957\end{array}$ & $\begin{array}{r}10,128,112 \\
9,833,563 \\
10,789,421 \\
11,455,009 \\
11,765,453 \\
11,866,111 \\
11,914,635 \\
11,936,600\end{array}$ & $\begin{array}{l}1914 \ldots . . \\
1915 \ldots . \\
1916 \ldots \\
1917 \ldots \\
1918 \ldots \\
1919 \ldots \\
1920 \ldots \\
\end{array}$ & $\begin{array}{l}4,595,128 \\
4,494,740 \\
4,499,321 \\
4,514,803 \\
4,603,841 \\
4,587,805 \\
4,381,794\end{array}$ & $\begin{array}{l}12,184,505 \\
12,171,452 \\
12,451,540 \\
12,382,236 \\
12,311,149 \\
12,491,427 \\
11,770,274\end{array}$ \\
\hline
\end{tabular}

TABLE 128.- United Kingdom-Number and kinds of CATTLE on the Islands of Jersey and Guernsey, June, 1920.

[Agricultural Statisties, 1920, Vol. LV, Part I.]

\begin{tabular}{|c|c|c|}
\hline Classification. & Jersey. & Guernsey. \\
\hline 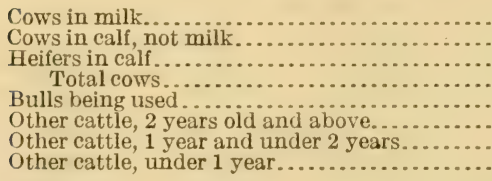 & $\begin{array}{r}4,966 \\
317 \\
1,000 \\
6,283 \\
126 \\
190 \\
1,674 \\
2,250\end{array}$ & $\begin{array}{r}2,837 \\
201 \\
327 \\
3,365 \\
89 \\
422 \\
1,045 \\
1,333\end{array}$ \\
\hline Total.... & 10,523 & 6,254 \\
\hline
\end{tabular}

TABLE 129.-United Kingdom-MILK PRODUCTION in Great Britain (England, Scotland, and Wales).

[Minister of Agriculture and Fisheries, Part III, 1919.]

\begin{tabular}{|c|c|c|c|c|c|}
\hline Year. & $\begin{array}{l}\text { Annual } \\
\text { yield } \\
\text { per cow. }\end{array}$ & $\begin{array}{l}\text { Annual } \\
\text { produc- } \\
\text { tion, less } \\
\text { milk for } \\
\text { calves. }\end{array}$ & Year. & $\begin{array}{c}\text { Annual } \\
\text { yield } \\
\text { per cow. }\end{array}$ & $\begin{array}{l}\text { Annual } \\
\text { produc- } \\
\text { tion, less } \\
\text { milk for } \\
\text { calves. }\end{array}$ \\
\hline $\begin{array}{l}1909-1913 \ldots . \\
1914 \ldots \ldots \\
1915 \ldots \ldots \\
1916 \ldots \ldots\end{array}$ & $\begin{array}{r}\text { Imperial } \\
\text { gallons. } \\
560 \\
575 \\
550 \\
525\end{array}$ & $\begin{array}{r}\text { Million } \\
\text { gallons. } \\
1,231 \\
1,253 \\
1,241 \\
1,164\end{array}$ & $\begin{array}{l}1917 \ldots \ldots \\
1918 \ldots \ldots \\
1919 \ldots \ldots\end{array}$ & $\begin{array}{r}\text { Imperial } \\
\text { gallons. } \\
495 \\
436 \\
470\end{array}$ & $\begin{array}{r}\text { Mfillion } \\
\text { gallons. } \\
1,086 \\
955 \\
1,085\end{array}$ \\
\hline
\end{tabular}

One imperial gallon (British) equals 1.2 U.S. gallons, or 10.32 pounds. 
TABLE 130.-United Kingdom-CONS UMPTION of DAIRY PRODDUCTS in 1919.

[Estimates, Minister for Food in National Food Journal, London, Mar. $10,1920$.

\begin{tabular}{|c|c|c|c|}
\hline Product. & $\begin{array}{c}\text { Total } \\
\text { consumption. }\end{array}$ & $\begin{array}{c}\text { Home } \\
\text { production. }\end{array}$ & Importation. \\
\hline $\begin{array}{l}\text { Butte } \\
\text { Chees }\end{array}$ & $\begin{array}{c}\text { Pounds. } \\
403,200,000 \\
324,800,000\end{array}$ & $\begin{array}{l}\text { Pounds. } \\
233,856,000 \\
97,440,000\end{array}$ & $\begin{array}{l}\text { Pounds. } \\
169,344,000 \\
227,360,000\end{array}$ \\
\hline
\end{tabular}

TABLE 131.-United Kingdom-Average ANNUAL CONSUMPTION of DAIRY PRODUCTS and MARGARIN from 1909 to 1913.

[Estimates in British Food Journal, July-October, 1919.]

\begin{tabular}{|c|c|c|c|}
\hline Product. & $\begin{array}{c}\text { Total } \\
\text { consumption. }\end{array}$ & $\begin{array}{c}\text { Home } \\
\text { production. }\end{array}$ & Importation. \\
\hline 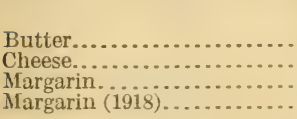 & $\begin{array}{c}\text { Pounds. } \\
748,160,000 \\
353,920,000 \\
264,320,000 \\
568,960,000\end{array}$ & $\begin{array}{r}\text { Pounds. } \\
\text { 282, } 056,320 \\
89,541,760 \\
134,274,560 \\
535,391,360\end{array}$ & $\begin{array}{l}\text { Pounds. } \\
466,103,680 \\
264,388,240 \\
130,045,440 \\
33,568,640\end{array}$ \\
\hline
\end{tabular}

TABLE 132.--United Kingdom-Average ANNUAL CONSUMPTION of DAIRY PRODUCTS and MARGARIN from 1909 to 1913.

[The Feeding of Nations, by E. H. Starling.]

\begin{tabular}{|c|c|c|}
\hline Product. & $\begin{array}{l}\text { Home produc- } \\
\text { tion and } \\
\text { importation. }\end{array}$ & $\begin{array}{l}\text { Per capita } \\
\text { consump- } \\
\text { tion. }\end{array}$ \\
\hline 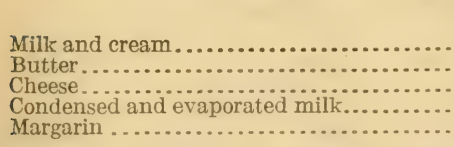 & $\begin{array}{c}\text { Pounds. } \\
9,920,700,000 \\
707,676,600 \\
324,076,200 \\
121,693,920 \\
261,465,560\end{array}$ & $\begin{array}{l}\text { Pounds. } \\
216 \\
115.38 \\
17.04 \\
2.61 \\
5.46\end{array}$ \\
\hline
\end{tabular}

1 The per capita consumption of butter and of cheese as shown in this talle is much less than that shown in Tables 4 and 5 . This taisle is based on the average for 5 years, 1909 to 1913 , while the figures in Tables 4 and 5 are based on a single year. There was a decrease in imports and in production during this period, hence a decrease in consumption.

Dairy products were estimated from 1908 census, and per capita consumption was obtained by using 46 million as average population for the period. 


\section{TABLE 133.-United Kingdom-IMPORTS and EXPORTS of BUTTER.}

[Trade and Navigation Reports; (YB.).]

\begin{tabular}{|c|c|c|c|c|c|}
\hline Year. & $\begin{array}{l}\text { General } \\
\text { jmports. }\end{array}$ & $\begin{array}{l}\text { Domestic } \\
\text { exports. }\end{array}$ & Year. & $\begin{array}{l}\text { General } \\
\text { imports. }\end{array}$ & $\begin{array}{l}\text { Domestic } \\
\text { exports. }\end{array}$ \\
\hline $\begin{array}{l}850 \ldots \\
860 \ldots\end{array}$ & $\begin{array}{r}\text { Pounds. } \\
35,823,648 \\
94,092,544 \\
129,831,520 \\
1260,546,160 \\
227,104,304 \\
378,363,792 \\
484,460,368 \\
481,901,504 \\
448,577,808\end{array}$ & $\begin{array}{c}\text { Pounds. } \\
6,791,568 \\
14,039,424 \\
6,443,136 \\
3,517,696 \\
2,745,120 \\
1,177,120 \\
1,035,328 \\
1,335,936 \\
1,270,304\end{array}$ & $\begin{array}{l}1913 \ldots \ldots \\
1914 \ldots \\
1915 \ldots \\
1916 \ldots \\
1917 \ldots \\
1918 \ldots \\
1919 \ldots \\
1920 \ldots \\
1921 \ldots\end{array}$ & $\begin{array}{c}\text { Pounds. } \\
463,571,136 \\
446,230,848 \\
431,631,760 \\
243,616,480 \\
202,329,792 \\
176,691,536 \\
2174,742,848 \\
2192,230,528 \\
2394,687,776\end{array}$ & $\begin{array}{r}\text { Pounds. } \\
1,220,240 \\
1,222,256 \\
1,178,352 \\
979,661 \\
264,208 \\
197,232 \\
252,752 \\
362,544 \\
1,103,200\end{array}$ \\
\hline
\end{tabular}

1 Including margarin for $1880 . \quad 2$ Preliminary figures.

\section{TABLE 134.-United Kingdom-IMPORTS and EXPORTS of CHEESE.}

[Trade and Navigation Reports; (YB.).]

\begin{tabular}{|c|c|c|c|c|c|}
\hline Year. & $\begin{array}{l}\text { General } \\
\text { imports. }\end{array}$ & $\begin{array}{l}\text { Domestic } \\
\text { exports. }\end{array}$ & Year. & $\begin{array}{l}\text { General } \\
\text { imports. }\end{array}$ & $\begin{array}{l}\text { Domestic } \\
\text { exports. }\end{array}$ \\
\hline $\begin{array}{l}1850 \ldots \ldots \\
1860 \ldots \ldots \\
1870 \ldots \ldots\end{array}$ & $\begin{array}{r}\text { Pounds. } \\
33,003,168 \\
65,327,696 \\
116,623,472 \\
198,911,664 \\
240,136,288 \\
303,058,336 \\
275,110,080 \\
263,012,512 \\
258,584,144\end{array}$ & $\begin{array}{r}\text { Pounds. } \\
968,016 \\
3,214,400 \\
2,821,728 \\
1,333,136 \\
1,367,632 \\
1,046,600 \\
911,008 \\
949,088 \\
956,704\end{array}$ & $\begin{array}{l}1913 \ldots \ldots \\
1914 \ldots \ldots \\
1915 \ldots \ldots \\
1916 \ldots \ldots \\
1917 \ldots \ldots \\
1918 \ldots \ldots \\
1919 \ldots \ldots \\
1920 \ldots \ldots \\
1921 \ldots \ldots\end{array}$ & \begin{tabular}{|c} 
Pounds. \\
$257,302,080$ \\
$272,592,768$ \\
$305,372,032$ \\
$291,661,888$ \\
$329,959,392$ \\
$263,131,901$ \\
$1237,968,080$ \\
$1308,029,120$ \\
$1314,918,688$
\end{tabular} & $\begin{array}{r}\text { Pounds. } \\
995,680 \\
804,832 \\
824,880 \\
985,824 \\
212,240 \\
70,448 \\
110,208 \\
454,018 \\
479,808\end{array}$ \\
\hline
\end{tabular}

1 Preliminary figures.

\section{TABLE 135.-United Kingdom-IMPORTS and EX- PORTS of CONDENSED AND EVAPORATED MILK.}

(Commerce and Trade Reports.)

\begin{tabular}{|c|c|c|c|c|c|}
\hline Year. & Imports. & Exports. & Year. & Imports. & Exports. \\
\hline $\begin{array}{l}1888 \ldots \ldots \\
1890 \ldots \ldots \\
1900 \ldots \ldots \\
1910 \ldots \ldots \\
1911 \ldots \ldots \\
1912 \ldots \ldots \\
1913 \ldots \ldots \\
1914 \ldots \ldots\end{array}$ & $\begin{array}{r}\text { Pounds. } \\
26,754,464 \\
34,370,560 \\
92,108,912 \\
105,974,000 \\
124,236,560 \\
132,082,832 \\
137,095,728 \\
133,525,728\end{array}$ & \begin{tabular}{r} 
Pounds. \\
\hdashline $6,886,992$ \\
$23,458,064$ \\
$49,194,432$ \\
$51,151,520$ \\
$52,651,424$ \\
$50,676,304$ \\
$7,146,960$
\end{tabular} & $\begin{array}{l}1915 . . \\
1916 . \\
1917 . \\
1918 . \\
1919 . \\
1920 . \\
1921 .\end{array}$ & $\begin{array}{c}\text { Pounds. } \\
170,970,016 \\
183,693,664 \\
184,255,344 \\
287,582,288 \\
1356,607,880 \\
1221,140,640 \\
1245,149,276\end{array}$ & $\begin{array}{r}\text { Pounds. } \\
37,628,192 \\
35,207,312 \\
14,271,264 \\
320,720 \\
1,437,296 \\
6,233,936 \\
14,205,936\end{array}$ \\
\hline
\end{tabular}

1 Preliminary figures.

Prior to 1888 condensed milk was included in "Other dairy products." 


\section{TOPICAL INDEX.}

Page.

Explanatory note.

Generalstatistics.

Butter-

consumption per capita, in various countries............. 5

production and exportation, by countries................... 7

standards of fat and moisture......................... 8

Butterfat receipts in creameries by months................. 9

Calf, quantity of milk to raise a......................... 9

Cheese, consumption per capita, by countries............... 6

Cow-testing associations, growth in various countries.......... 6

Food, normal, for United Kingdom...................... 9

Milk-

and cream, weight per gallon, with varying fat content...... 8

consumption per capita, by countries.................. 5

solids in various dairy products........................ 7

to raise a calf ...................................... 9

yield per cow, by countries........................... 5

Population of various countries......................... 4

United States....................................... 10

Accredited herds....................................... 18

Balance of trade with foreign countries, in dairy products..... 40,41

Bull associations................................... 16

Butter-

creamery, in storage, by months..................... 28

exports and imports............................. 29, 40,41

factories, number of ............................ 25

factory and farm production, by years.................. 25

from whey, production, by months................... 21, 25

production in factories, by months..................... 21

production in factories, by States..................... 23, 24

production on farms, by States........................ 27

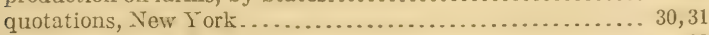

quotations, San Francisco............................. 29

receipts, New York, Chicago, San Francisco............... 28

renovated, New York quotations......................... 36

renovated, production and export..................... 22

Butterfat-

delivered to creameries............................... 9

from advanced-register cows........................ 14

Buttermilk-

condensed or evaporated.............................. 37

dried or powdered.................................... 26, 38

Casein-

production from skim milk and buttermilk................ 26

production, importation, and prices..................... 37

Cattle-

exported......................................... 10

imported......................................... 11, 14

number, by breeds.................................. 10, 12

number of dairy cows, on farms ...................... 11, 12,27

number of other cattle on farms, by years............... 11 
United States-Continued.

Cattle-Continued.

purebred-

Page

butterfat production, advanced register.................. 14

imported....................................... 11

number on farms, by States ....................... $\quad 12$

registered, by years-

Ayrshires........................................ 14

Guernseys.................................. 15

Jerseys.......................................... 15

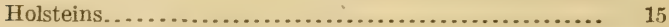

tuberculin-tested, by breeds. ......................... 18

Cheese-

A merican Cheddar............................. 21,31,35

New York quotations............................. 34

production, from whole milk and skim milk............. 21, 31

brick and Münster, production....................... 21, 31

cottage, pot, and baker's, production.............. 21, 23, 24, 32

cream and Neufchâtel, production.................... 21, 31

damestic Swiss-

Chicago quotations................................ 34

production, by months and years................. 21,31

production, by States............................. $\quad 32$

filled, production and export........................... 35

imports and exports............................. 33, 40,41

imports from France, Greece, Italy, and Sivitzerland....... 33

in storage, by months............................ 35

Italian varieties, production in United States. . . . . . . . . . 21,31

Limburger, production............................ 21,31 production-

by kinds...................................... 21, 31

farm and factory .................................. $\quad 32$

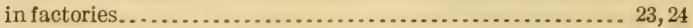

on farms...................................... 27, 32

Condensed milk, evaporated and dried milk, etc.-

buttermilk, powdered or condensed, production.... 23, 24, 26, 37, 38 condensed milk, skimmed and unskimmed, sweetened and un-

sweetened, production ........................... 22

condensed and evaporated milk, imports and exports . ... 36, 40, 41 condensed and evaporated milk, production........ 22, 23, 24, 36, 37 evaporated milk with foreign fats, production.......... 22, 23,24 factories in operation............................. $\quad 37$

malted milk, production.......................... 26, 38

powdered milk, whole or skimmed, production ........... 26,38

powdered cream, production......................... 26

sterilized milk, canned, production................ 22, 23, 24, 36

Cost of production of milk............................. 20

Cow-testing associations-

growth before and after war.......................... $\quad 6$

number in United States............................ $\quad 16$

yearly records in .................................. 16

Creameries-

butterfat delivered to, in each month.................. 9

number in United States........................... 25 
- United States-Continued.

Dairy products made - Page.

in factories, by months............................ 21, 22

in factories, by States............................... 23,24

on farms, by States. . ................................ 27

Ice-cream production, by months and years............... $\quad 39$

Milk-

distribution by uses............................... 17

production-

cost of . . . . . .

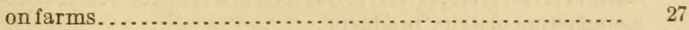

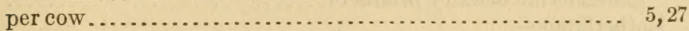

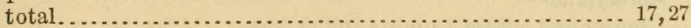

received in Philadelphia, New York, and Boston........... 19

retail price, New York............................... 19

wholesale price, Chicago................................ 18

(See also Condensed milk, etc.)

Milk sugar-

production, by months............................ $\quad 26$

production and importation, by years................... 38

Oleomargarin, production and exportation................ 34

Silos, number and capacity .......................... 17

Tuberculin-tested cattle, by breeds........................ 18

Whey butter, production. . . . . . . . . . . . . . . . .

Foreign countries:

Argentina-

butter and cheese, imports and exports...................

butter and cheese, production and consumption.............

cattle, number and character...........................

Australia-

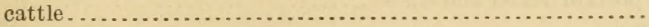

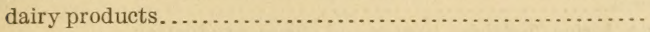

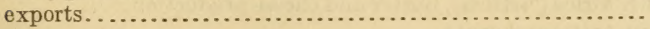

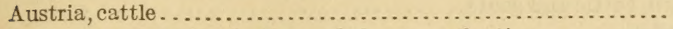

British South Africa, butter and cheese production............

Canada-

butter and cheese, imports and exports..................

butter and cheese production...........................

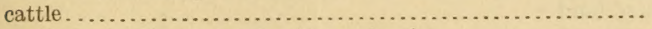

condensed and powdered-milk production...................

exports of dairy products................................

Denmark-

butter imports and exports............................

butter quotations.....................................

cattle ..............................................

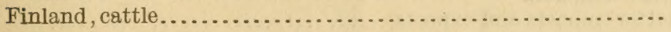

France-

cattle ............................................. 51

cheese exported to United States.......................... 33

i mports and exports............................... 51,52

Germany-

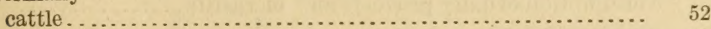

consumption of butter and cheese ....................... 52

i mports and exports................................ 53

production of milk, butter, and cheese.................. 53 
Foreign countries-Continued.

Page.

Greece, cheese exported to United States .................. 33

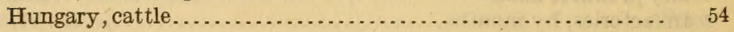

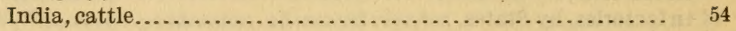

Italy-

cattle, buffaloes, sheep, and goats.................. 54

cheese exported to United States...................... 33

i mports and exports............................. 55

Japan-

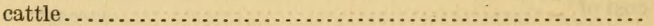

manufacture of dairy products. ................... 56

Netherlands-

butter and cheese, imports and exports.............. 58

butter production and consumption.................. 56,57

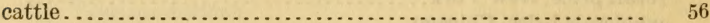

New Zealand-

cattle.

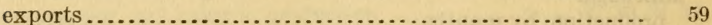

Norway, cattle and reindeer...................... 59

Pan American countries-

butter imports and exports.......................... 42

cheese imports and exports....................... 42

condensed-milk imports............................ 43

Poland-See Russia.

Roumania, cattle................................. 60

Russia-

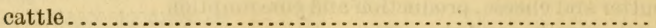

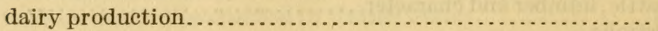

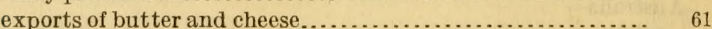

Siberia.......................................... 60,61

Scuth Africa (British), butter and cheese production......... 61

Spain, cattle and goats............................... 61

Sweden-

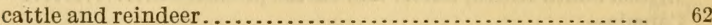

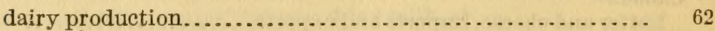

imports and exports.............................. 63

Switzerland-

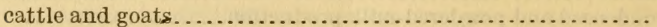

cheese exported to United States.......................

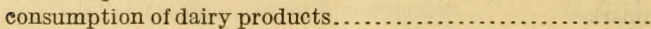

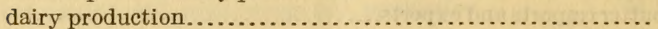

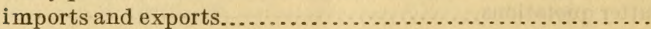

United Kingdom-

butter imports and exports.......................... 68

cattle and cows in kingdom. ......................... 66

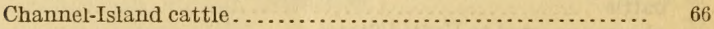

cheese imports and exports............................. 68

condensed and evaporated milk, imports and exports...... 68

consumption of dairy products and margarin............ 67

milk production................................. 66 



\section{LIBRARY OF CONGRESS}

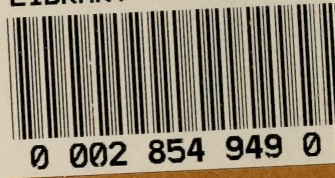

